\title{
Electron volt neutron spectrometers
}

\author{
A. Pietropaolo*, R. Senesi \\ Universitá degli Studi di Roma Tor Vergata, Dipartimento di Fisica and Centro NAST (Nanoscienze-Nanotecnologie-Strumentazione), via della Ricerca Scientifica \\ 1, 00133 Roma, Italy
}

\section{A R T I C L E I N F O}

\section{Article history:}

Accepted 21 April 2011

Available online 21 July 2011

editor: D.L. Mills

\section{Keywords:}

Epithermal neutrons

Neutron instrumentation

Neutron time of flight spectrometers

Epithermal neutron detectors

Electron volt neutron energy selectors

\begin{abstract}
A B S T R A C T
The advent of pulsed neutron sources has made available intense fluxes of epithermal neutrons (500 meV $\leq E \leq 100 \mathrm{eV}$ ). The possibility to open new investigations on condensed matter with eV neutron scattering techniques, is related to the development of methods, concepts and devices that drive, or are inspired by, emerging studies at this energy scale. Electron volt spectrometers have undergone continuous improvements since the construction of the first prototype instruments, but in the last decade major breakthroughs have been accomplished in terms of resolution and counting statistics, leading, for example, to the direct measurement of the proton 3-D Born-Oppenheimer potential in any material, or to quantitatively probe nuclear quantum effects in hydrogen bonded systems. This paper reports on the most effective methods and concepts for energy analysis and detection, as well as devices for the optimization of electron volt spectrometers for different applications. This is set in the context of the progress made up to date in instrument development. Starting from early stages of development of the technique, particular emphasis will be given to the Vesuvio eV spectrometer at the ISIS neutron source, the first spectrometer where extensive scientific, as well as research and development programmes have been carried out. The potential offered by this type of instrumentation, from single particle excitations to momentum distribution studies, is then put in perspective into the emerging fields of eV spectroscopy applied to cultural heritages and neutron irradiation effects in electronics.
\end{abstract}

(C) 2011 Elsevier B.V. All rights reserved.

\section{Contents}

1. Introduction 46

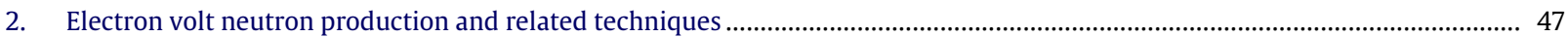

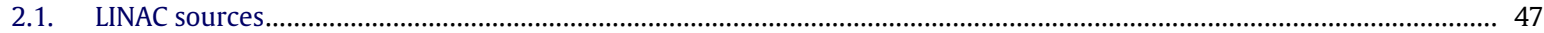

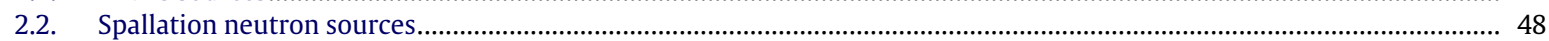

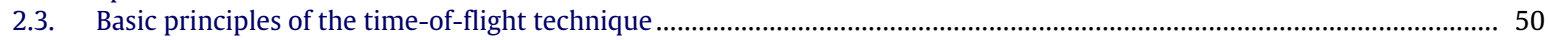

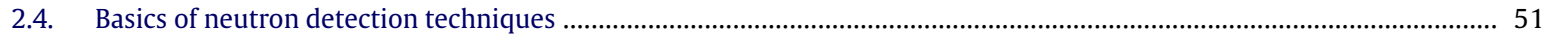

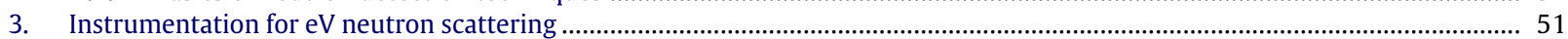

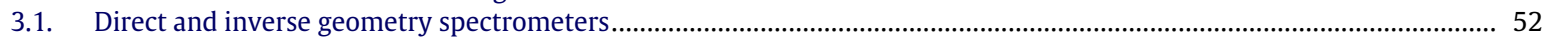

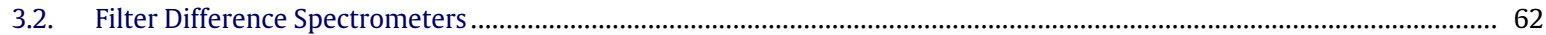

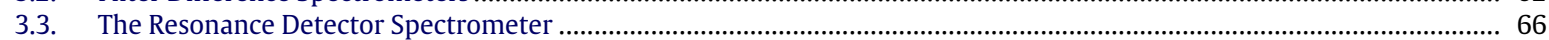

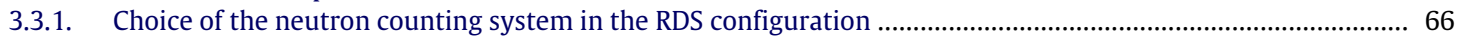

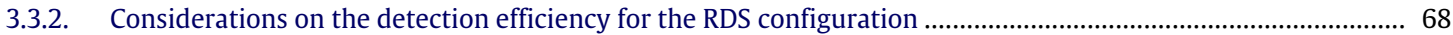

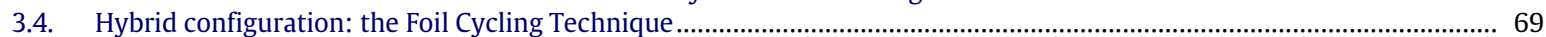

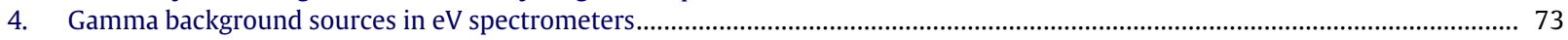

\footnotetext{
* Corresponding author. Tel.: +39 0672594549; fax: +39 062023507.

E-mail address: antonino.pietropaolo@roma2.infn.it (A. Pietropaolo).
} 


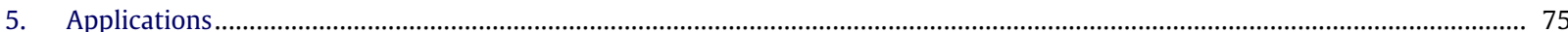

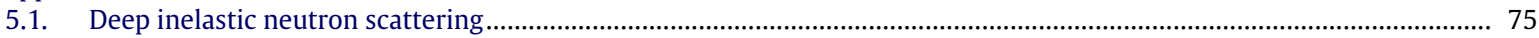

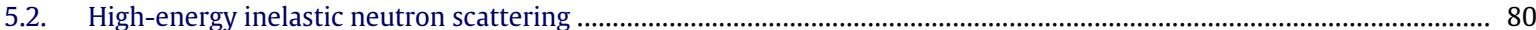

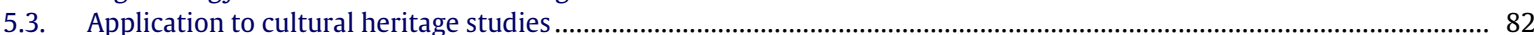

5.4. Exploiting the edge of the spallation neutron spectrum: the Chip Irradiation case ....................................................... 82

6. Conclusions. 84

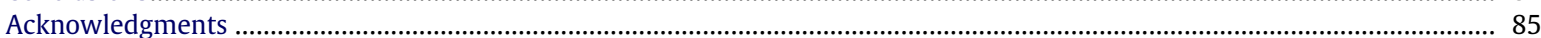

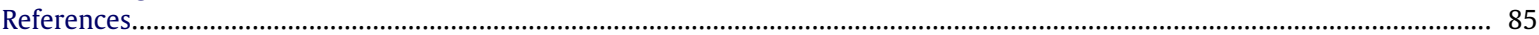

\section{Introduction}

Neutron scattering beam lines located at spallation or photo-production neutron sources, benefit from intense fluxes of thermal and epithermal (above $400 \mathrm{meV}$ ) neutrons that represent more than $50 \%$ of the total spectral intensity [1-5]. In particular, on the Vesuvio spectrometer [6] at the ISIS neutron facility [7], the incident neutron flux at the sample position in the energy range $0.4 \mathrm{eV} \leq E_{0} \leq 100 \mathrm{keV}$, is about $6.5 \times 10^{6} \mathrm{n} \mathrm{cm}^{-2} \mathrm{~s}^{-1}$ [1]. This represents an incident flux comparable with that in the thermal region available for inelastic spectrometers at both continuous and pulsed sources [8]. Soon after the design and construction of accelerator-based neutron sources [9], the original proposals of neutron spectroscopy above $1 \mathrm{eV}$ for the study of condensed matter [10-13], were brought into reality. Indeed, prototype eV spectrometers were set up soon after the construction of spallation and photo-production based pulsed neutron sources [14-20] to exploit the time of flight technique [21] for the kinematical reconstruction of the scattering events. These followed a pioneering measurement in the multi-eV range carried out at the Dubna pulsed reactor by Samosvat et al. [22], whose inspiring potential for further studies can be appreciated in Fig. 1 below.

In fact, it appeared that vibrational, rotational, and perhaps electronic transitions in molecules could be excited, with measurable probability, through electron volt neutron-nucleus collisions. This motivated experimental studies on molecular systems with pulsed sources and neutron time of flight techniques in combination with resonant neutron capture detectors [22]. These measurements aimed at general studies of excitations by inelastic scattering using eV neutrons. As an example, Fig. 2 reports the energy spectrum of the inelastically scattered neutrons from benzene [16].

These experiments demonstrated the potential of eV spectroscopy: while the most famous proposal for eV neutron scattering was addressed to the determination of the Bose condensate fraction in superfluid helium [12], the pioneering measurements, where the bases of the technique were introduced, have been carried out on molecular systems. While the experimental and theoretical programme on eV neutron scattering from monoatomic quantum liquids had remarkable developments in the late eighties and nineties [23], the activity on molecular, and especially hydrogen-bonded systems, received a less participated attention. The last decade has shown how the breakthroughs in methods, concepts and devices have allowed eV spectrometers, and in particular Vesuvio at the ISIS source (UK), to reach an unprecedented capability for the study of proton momentum distribution with scientific results going beyond the expectation of the initial proposals. The techniques and methods set up in the last decade have allowed a more than twofold improvement in the instrumental resolution, and a factor of ten improvement in signal to background ratio. These opened up new perspectives for eV spectrometers towards high momentum-high energy studies in the deep inelastic neutron scattering regime (DINS), such as the measurement of the interatomic Born-Oppenheimer and effective potentials in molecules [24-26]. On the other hand, the study of low- $q$ inelastic scattering with $\mathrm{eV}$ neutrons has always been considered of great interest [27-29] and, thanks to the above mentioned improvements, its potential has been glimpsed very recently by the set up of dedicated instrumentation [30,31]. Interdisciplinary applications of eV spectrometers have been as well developed in the last decade, such as the neutron resonance capture analysis of materials, with special application to complex artefacts of historical/cultural interest [32-34]. The experimental and theoretical scientific programmes led the motivations to these achievements, but the substantial contributions have to be found in the continuous developments in detection concepts and methods, and related devices, as well as to energy analysis techniques. The latter are peculiar to eV spectroscopy, in that in this energy range the techniques typical of nuclear physics do not operate, nor the common methods used for detection and energy analysis in thermal neutron scattering. We stress that while eV neutron spectroscopy aims at a microscopic description of condensed matter, sharing the scope of thermal neutron spectroscopy, both energy selection and detection are based on different principles and methodologies with respect to the thermal range. For example, the concepts of crystal monochromator or mechanical velocity selector [35-38] have to be abandoned, because of the loss of efficiency and/or resolution above $1 \mathrm{eV}$. At the same time the concept of gas proportional counter detector, i.e. the most used neutron detector [39], cannot be applied due to the loss of efficiency and time resolution, and even the neutron counting procedure has to be modified, by exploiting radiative neutron capture. As it has been done in the case of reviewing the scientific programme on the experimental, theoretical and interpretation results on momentum distribution studies using inelastic eV neutron scattering, that has been the subject of monographs [23] and reviews [40,41], a broad overview of techniques, methods, and devices instrumental to the advances in electron volt spectrometers motivated the present work. In our view, eV neutron spectroscopy is given by the concerted contributions of the scientific applications for the investigation of materials, and the enabling techniques and devices. The work described here highlights the most relevant techniques and methods, and is intended as a reference for the next generation of electron volt neutron spectrometers. 


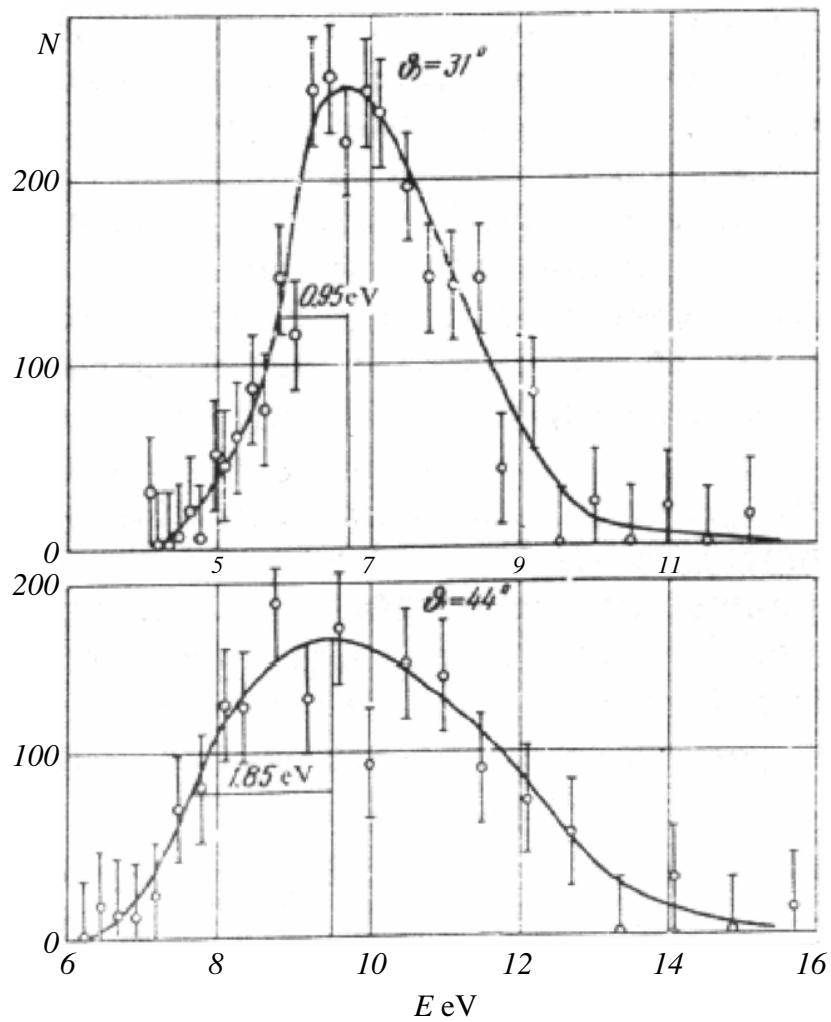

Fig. 1. Energy spectra for eV scattering from liquid water, showing the proton recoil line shape for two scattering angles. Source: From Ref. [22].

\section{Electron volt neutron production and related techniques}

Intense fluxes of electron volt pulsed neutrons are mainly produced by accelerated beams of both electrons and protons. Electron-induced neutron production is mainly performed at linear accelerators (LINAC) sources and exploit the electromagnetic interactions of electrons in matter. Spallation neutron sources rely upon the interactions of high energy projectiles (protons) with heavy metals target nuclei [42-46]. As generally the projectile energy is about $1 \mathrm{GeV}$, the strong interactions are mostly responsible for the neutron production, although a series of mechanisms are involved as briefly discussed below.

\subsection{LINAC sources}

LINACs accelerate electrons emitted by a electron "gun" up to energies in the order of $100 \mathrm{MeV}$. The electrons, packed into bunches, are then directed towards a target material (e.g. uranium) where they produce neutrons in a two-step electromagnetic process: (1) production of bremsstrahlung radiation [47], (2) photo-neutron $(\gamma, n)$ production [48]. The whole process is not highly efficient and only a few percent of the incident electrons produce a neutron. Furthermore, a few thousand of $\mathrm{MeV}$ of energy are dissipated for each neutron produced, with related problems of heat removal [49]. Fig. 3 schematically shows the two-step process involved in the neutron production, while Fig. 4 shows a sketch of a LINAC-based neutron source.

The GELINA neutron source [50], for example, produces electron beams of $100 \mathrm{MeV}$ average energy, $10 \mathrm{~ns}$ pulse length, $800 \mathrm{~Hz}$ repetition rate, $12 \mathrm{~A}$ peak and $100 \mathrm{~A}$ average current. With a post-acceleration pulse compression system, the electron pulse width can be reduced to approximately $1 \mathrm{~ns}$ (FWHM) while preserving the current, resulting in a peak current of $120 \mathrm{~A}$. The accelerated electrons produce bremsstrahlung in an uranium target which in turn, by photonuclear reactions, produces neutrons. Within a $1 \mathrm{~ns}$ pulse a peak neutron production of $4.3 \times 10^{10}$ neutrons is achieved (average intensity of $3.4 \times 10^{13} \mathrm{n} \mathrm{s}^{-1}$ ). The neutron energy distribution emitted by the target ranges from sub-thermal to about $20 \mathrm{MeV}$, with a peak at 1-2 MeV. To have a significant number of neutrons in the energy range below $100 \mathrm{keV}$, a hydrogen-rich moderator is used. The partially moderated neutrons have an approximate $1 / E$ energy dependence plus a Maxwellian peak at thermal energy. By using collimators and shadow bars, moderated or unmoderated neutron beams are selected for the twelve neutron flight paths.

In what follows we will focus our attention on spallation neutron sources and the related instrumentation. 


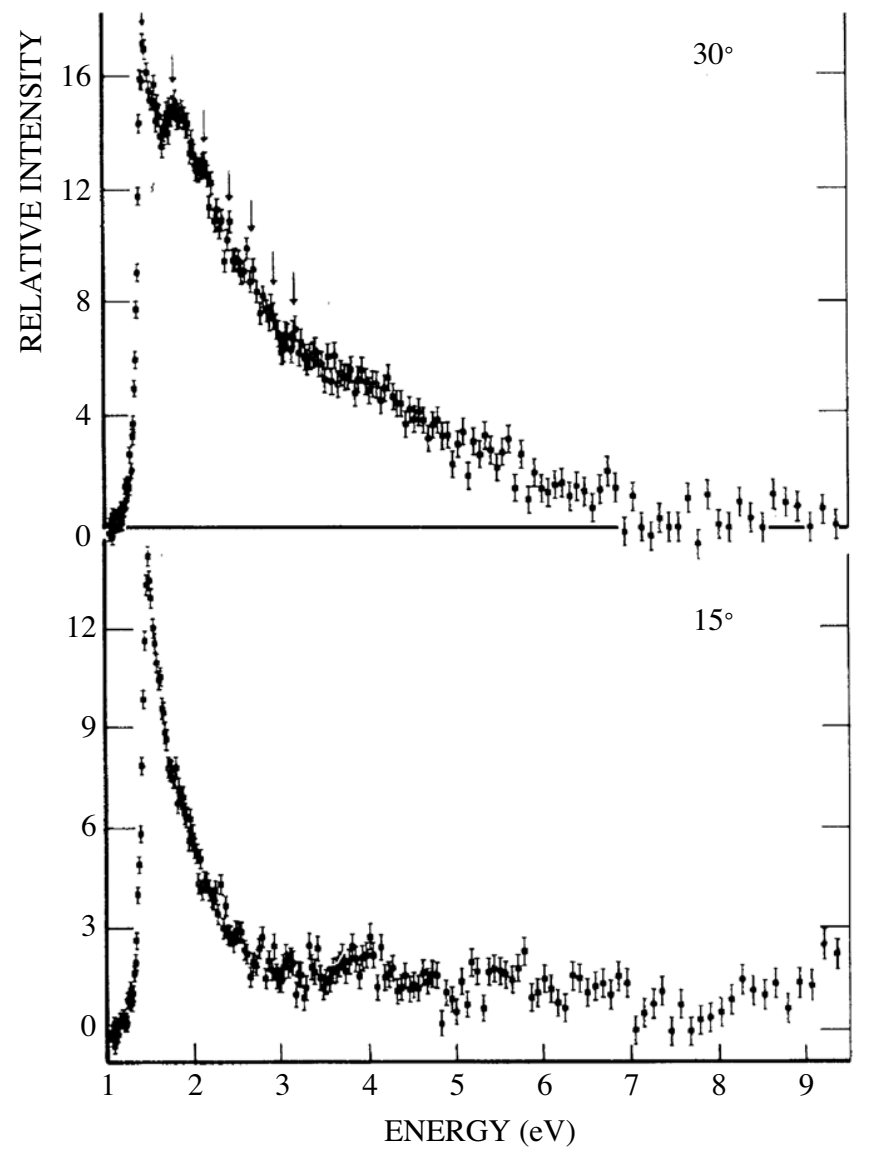

Fig. 2. Incident-energy dependent spectrum of the inelastically scattered neutrons from benzene; the difference between the incident energy and the final energy $(1.46 \mathrm{eV})$ fixed by the indium resonance analyser is the excitation energy. Vertical arrows mark the fundamental energy and overtones of the C-H vibration.

Source: From Ref. [16].

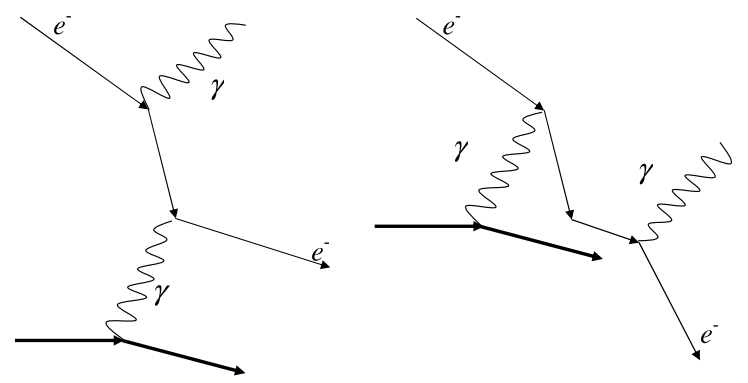

Fig. 3. Feynman's diagrams of the bremsstrahlung radiation process.

\subsection{Spallation neutron sources}

In the proton-induced neutron production, negative hydrogen ions are accelerated in a LINAC up to several tens of $\mathrm{MeV}$. Before the injection into the synchrotron, the electrons of the $\mathrm{H}^{-}$ions are removed by a stripper, the protons being accelerated and packed into bunches with energy up to about $10^{9} \mathrm{eV}$. The proton bunches are then directed in short bursts towards a metallic target (e.g. tungsten or lead) where neutrons are produced by spallation reactions, the neutron yield (i.e. the number or neutrons produced per proton) depending on proton energy and on the target material and geometry [44]. Detailed description of the spallation reactions can be found in Refs. [51-57].

Fig. 5 shows a schematic of the hadronic reactions involved in the spallation process, while Fig. 6 represents a schematic layout of the ISIS spallation source at the Rutherford Appleton Laboratory (RAL) in United Kingdom, briefly described in the following. 


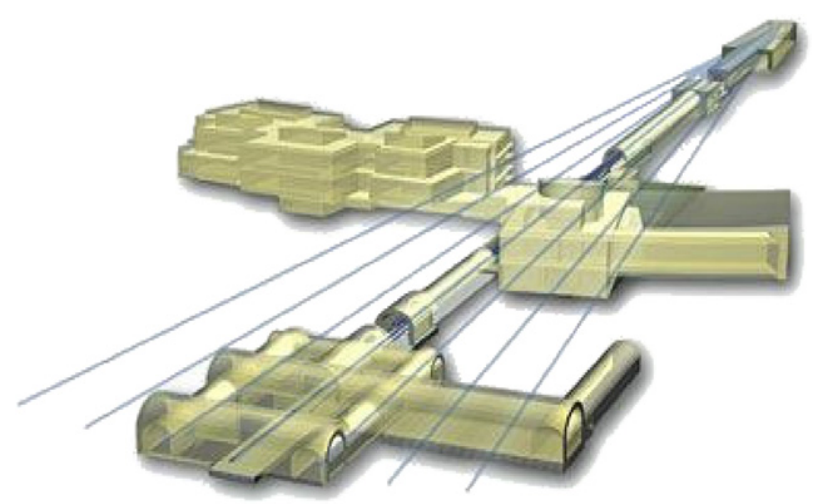

Fig. 4. Schematic picture of a Linac-based neutron source.

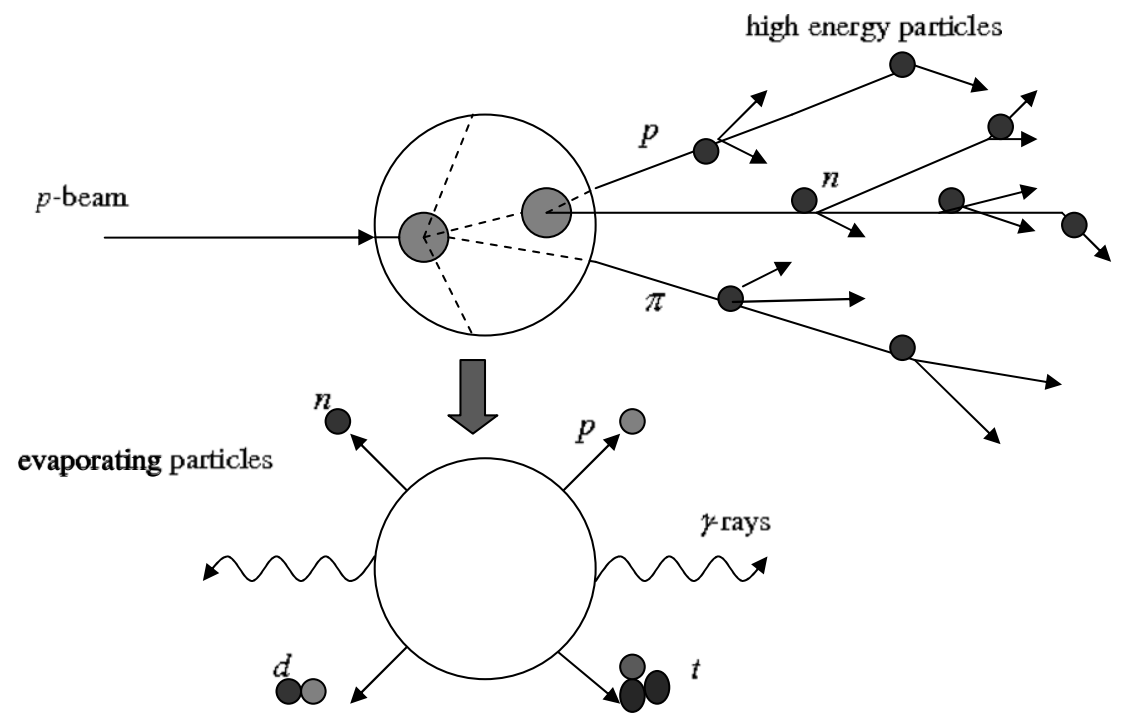

low energy particles

Fig. 5. Schematic drawing representing the possible nuclear processes involved in the spallation reaction.

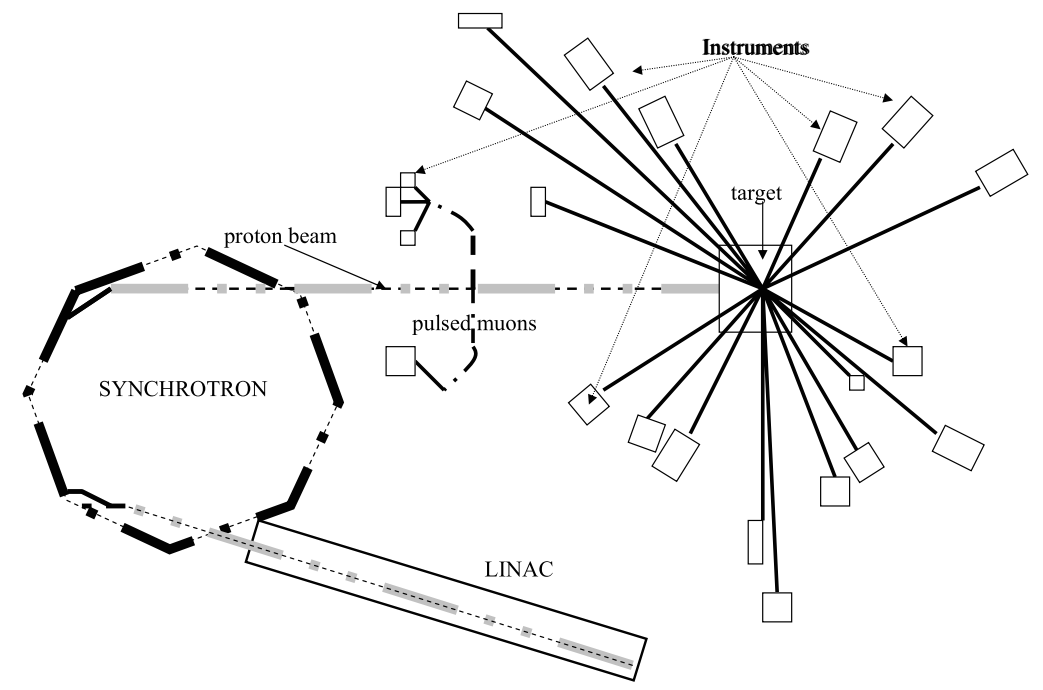

Fig. 6. Schematic layout of the ISIS spallation source with the corresponding beam lines arrangement. 


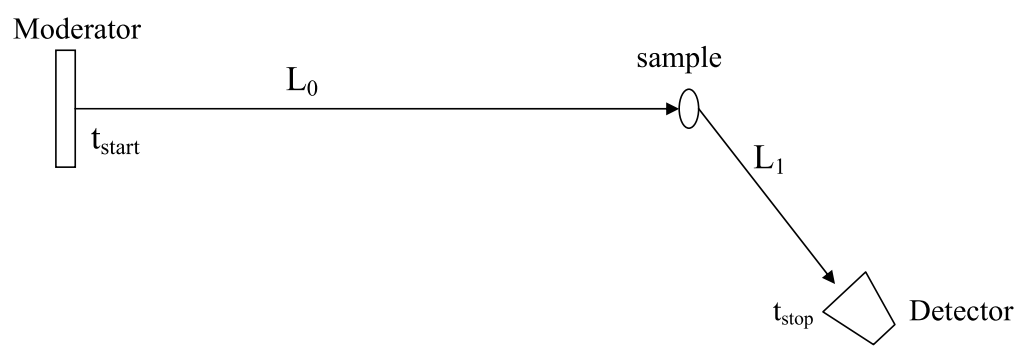

Fig. 7. Schematics of the neutron time of flight principle.

An ion source produces $\mathrm{H}^{-}$ions at $17 \mathrm{keV}$ energy in $200 \mu \mathrm{s}$ long pulses to form a beam that is then curved through a $90^{\circ}$ magnet to remove any electrons. The $\mathrm{H}^{-}$ions then reach an energy of $35 \mathrm{keV}$ across a DC acceleration gap, and are focused and directed into the radio frequency quadrupole (RFQ) accelerator, operating at $665 \mathrm{keV}$ and $202.5 \mathrm{MHz}$. Inside the $\mathrm{RFQ}$ four specially shaped electrodes produce an alternating gradient quadrupole electric field for focusing and acceleration. Discrete bunches of $\mathrm{H}^{-}$ions $4.94 \mathrm{~ns}$ apart are passed into the LINAC, where they are further accelerated up to $70 \mathrm{MeV}$. Before the injection into the synchrotron, the electrons of the $\mathrm{H}^{-}$ions are removed by the stripper. In the synchrotron the protons are then accelerated up to $800 \mathrm{MeV}$, with a bunch rate of $50 \mathrm{~Hz}$. The neutron pulse produced by spallation is very short (typically few hundreds of nanoseconds) and peaked at high energy. A complex system of moderators, reflectors and coolers surrounds the spallation target that is made of a set of thin tantalum sheets. The purpose of the moderators is to scatter the fast leakage neutrons $(1 \mathrm{MeV})$ from the target, slowing them down to the energies required for the neutron instruments (typically between about $1 \mathrm{meV}$ and $100 \mathrm{eV}$ ), maintaining the time correlation with the proton beam. This allows for using the time of flight (henceforth called TOF) technique for the kinematic reconstruction of the scattering process (i.e. to define energy and wave vector transfer). In the following section a brief description of the TOF technique is presented.

Since August 2009, the second target station (TS2) became operational at ISIS. One in every five pulses is now diverted to TS2, whilst the remainder continue to the existing target. With the accelerators continuing to operate at $50 \mathrm{~Hz}$ ( 50 proton pulses per second), this result in a $60 \mu \mathrm{A}$ proton beam operating at $10 \mathrm{~Hz}$.

\subsection{Basic principles of the time-of-flight technique}

The total neutron TOF, $t$, is the time a neutron takes to travel from the moderator to the detector. It can be written as:

$$
t=t_{0}+\frac{L_{0}}{v_{0}}+\frac{L_{1}}{v_{1}}
$$

where $t_{0}$ is a fixed electronic time delay, $L_{0}$ and $L_{1}$ are the (known) incident and scattering flight paths of the instrument, while $v_{0}$ and $v_{1}$ are the initial and final neutron velocities, respectively. The energy transfer is:

$$
\hbar \omega=m \frac{\left(v_{0}^{2}-v_{1}^{2}\right)}{2}
$$

and the momentum transfer:

$$
\hbar q=m\left(v_{0}^{2}+v_{1}^{2}-2 v_{0} v_{1} \cos \vartheta\right)^{1 / 2}
$$

where $\vartheta$ is the scattering angle.

Fig. 7 represents a schematic layout of a TOF instrument.

The TOF technique requires the knowledge of $L_{0}, L_{1}$, the scattering angle $\vartheta$ and of the initial or the final neutron energy $\left(E_{0}\right.$ or $\left.E_{1}\right)$ to reconstruct the kinematics of the scattering process. By knowing the initial (final) energy, the total neutron TOF allows for the measurement of the final (initial) one, while the detector's angular position, $\vartheta$, allows for the determination of the wave vector transfer, $q$.

The TOF acquisition chain can briefly be described as follows: before the proton bunch impinges on the heavy metal spallation target, a proton beam monitor, placed close to the target, triggers the opening of a time gate $\left(t=t_{\text {start }}=0\right)$ of fixed duration ( $\Delta t \simeq 20 \mathrm{~ms}$ at ISIS-TS1). During $\Delta t$ the acquisition electronics of an instrument is enabled to process the signals provided by the neutron detection system (neutron counters). Each signal is stored in a time channel of a Time to Digital Converter (TDC), its value being the time difference between the initial time of the gate $t_{\text {start }}$ (approximatively corresponding to the instant the neutron leaves the moderator) and the detection instant $\left(t_{\text {stop }}\right)$. A fixed time delay $t_{0}$ (see Eq. (1)) is typically provided, allowing for the recovery of the detectors of all instruments from the saturation induced by the so-called " $\gamma$-flash", produced in the spallation process [58-60] that will be recalled later in this review. 
Table 1

List of the nuclear reactions useful for neutron detection.
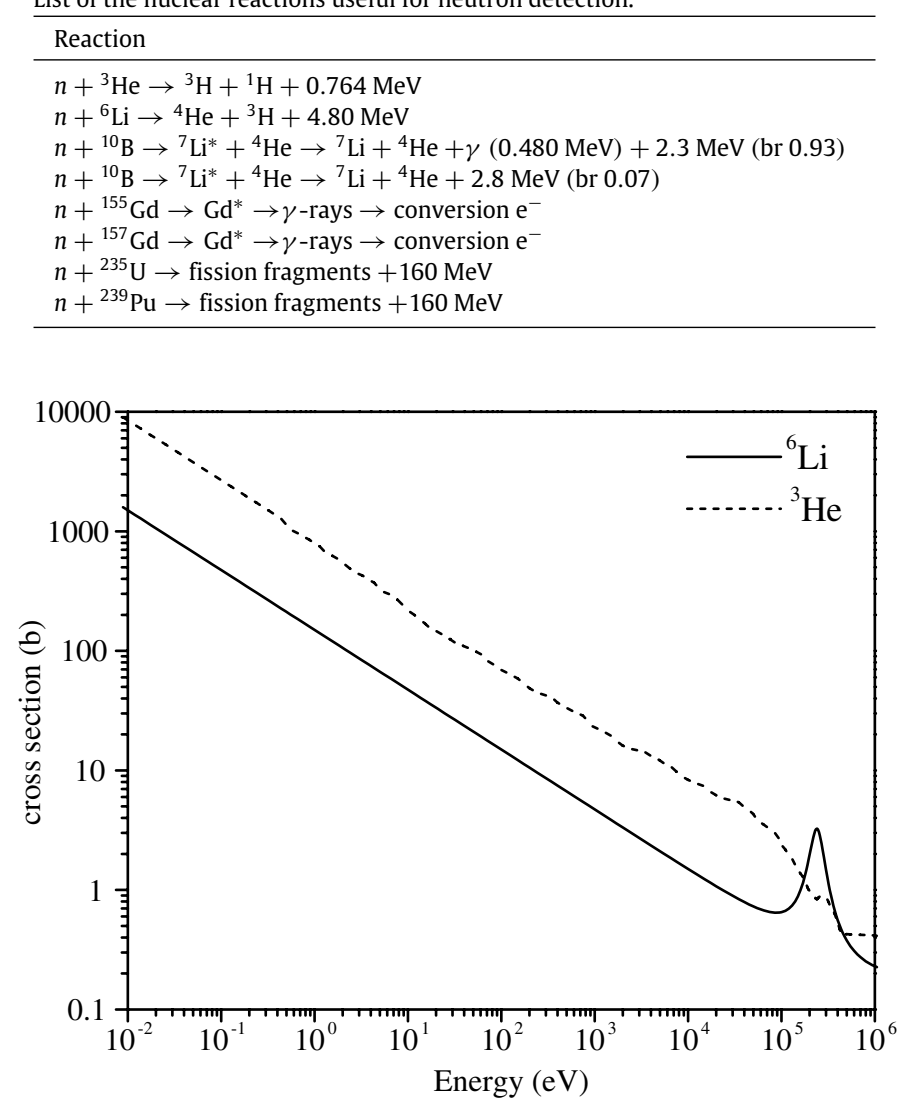

Fig. 8. Neutron absorption cross section of ${ }^{3} \mathrm{He}$ (dashed line) and ${ }^{6} \mathrm{Li}$ (continuous line) [61].

\subsection{Basics of neutron detection techniques}

The detection of the neutrons for condensed matter studies (from ultra-cold to epithermal) is based on nuclear reactions, where a neutron is absorbed and charged particles are produced and then detected by the energy they release in materials.

The main nuclear reactions used for neutron detection are listed in Table 1 . As the $Q$-values of all the listed reactions are positive, these are triggered by neutrons in the meV-eV range (for example) always releasing almost the same energy in the detector. The direct spectroscopic measurement of the neutron energy (in the range of interest for neutron scattering) based on these reactions is not possible, because the intrinsic kinetic information on the neutron is lost in the large amount of energy released in the reaction. To perform a neutron scattering measurement it is necessary to select neutron energy before counting. Once the charged particles have been produced by the one of the nuclear reactions listed in Table 1, they can be detected with gaseous, scintillation or solid state detectors.

In the majority of the reactions in Table 1 , the main problem to be faced for epithermal neutrons detection, is the $1 / v$ dependence of the reaction cross sections, as indicated in Fig. 8 for the ${ }^{3} \mathrm{He}$ and ${ }^{6} \mathrm{Li}$, respectively. In the $1-100 \mathrm{eV}$ range, for example, a way to overcome the problem of the energy dependent detection efficiency will be described in some details in the next section, especially for inverse geometry TOF instruments. At the time of writing, the use of ${ }^{3} \mathrm{He}$, with its intrinsically low noise as neutron converter, is severely restricted due to the shortage of commercial ${ }^{3}$ He worldwide, and a general effort is currently carried out for ${ }^{3} \mathrm{He}$ replacement in neutron detectors.

\section{Instrumentation for $\mathrm{eV}$ neutron scattering}

As already mentioned before, all neutron beam lines for both elastic and inelastic scattering experiments at spallation neutron sources use the TOF technique to resolve diffraction patterns from different Miller indexes [62,63] or to record inelastic spectra at different $\hbar \omega$ and $q[64,65]$. In what follows, we will briefly describe direct and inverse geometry instruments, eventually focusing our discussion on the inverse geometry spectrometers, where eV neutron scattering is widely and more effectively performed for condensed matter investigations $[21,66]$. 
Table 2

Energy component of the resolution for the different foils: $\Delta E_{1}$ represents the full width at half maximum (FWHM) of the experimental Voigt line shape at room temperature. In the case of $U$ the value at a temperature $T=77 \mathrm{~K}$ is also reported (indicated with the prefix $\left({ }^{*}\right)$ ).

\begin{tabular}{lcclcl}
\hline Filter & $E_{1}(\mathrm{eV})$ & $\Delta E_{1}(\mathrm{meV})$ & Filter & $E_{1}(\mathrm{eV})$ & $\Delta E_{1}(\mathrm{meV})$ \\
\hline${ }^{149} \mathrm{Sm}$ & 0.872 & 83 & ${ }^{150} \mathrm{Sm}$ & 20.7 & 261 \\
${ }^{240} \mathrm{Pu}$ & 1.06 & 56 & ${ }^{238} \mathrm{U}$ & 20.9 & 177 \\
${ }^{185} \mathrm{Re}$ & 2.16 & 58 & ${ }^{238} \mathrm{U}$ & 36.6 & 242 \\
${ }^{242} \mathrm{Pu}$ & 2.67 & 71 & ${ }^{238} \mathrm{U}$ & 66.0 & 320 \\
${ }^{197} \mathrm{Au}$ & 4.91 & 182 & ${ }^{139} \mathrm{La}$ & 72.1 & 436 \\
${ }^{238} \mathrm{U}$ & 6.67 & 103 & ${ }^{168} \mathrm{Er}$ & 79.7 & 120 \\
${ }^{238} \mathrm{U}$ & 6.67 & $66 *$ & & & 410 \\
${ }^{187} \mathrm{Os}$ & 12.7 & 100 & ${ }^{238} \mathrm{U}$ & 102.6 & 410 \\
\hline
\end{tabular}

\subsection{Direct and inverse geometry spectrometers}

Direct geometry spectrometers monochromate the incident neutron beam (i.e. incident neutrons lying within a narrow range of energies are selected), while inverse geometry instruments monochromate the scattered beam [67]. The TOF technique is then used to measure the energy of the scattered neutron, in the first case, or the incident neutron energy, in the second one. There are three methods of neutron energy selection of common use: (1) a neutron is reflected from a single crystal such as graphite $[68,69]$ : the angle of reflection determines the energy of the reflected neutrons as described by the Bragg's law. Unfortunately the reflectivity of crystals decreases rapidly at high energies. This implies that this is not a suitable method to perform measurements with $q>20 \AA^{-1}$, in either direct or indirect geometry instruments. (2) A chopper is placed in the incident beam [70,71]: this transmits neutrons only for a short opening time, which is phased to the source pulse and hence only neutrons within a narrow velocity band are selected in the incident beam. The maximum energy [21] and hence the maximum energy transfer attainable in this way is limited by the maximum rotational speed of the chopper to typically about $2 \mathrm{eV}$, with a corresponding maximum $q$ of about $30 \AA^{-1}$. Recently, attempts are under way to test the capability of chopper spectrometers to provide efficient energy selection up to $100 \mathrm{eV} \mathrm{[72].} \mathrm{(3)} \mathrm{A} \mathrm{foil,} \mathrm{made} \mathrm{of} \mathrm{a}$ material with narrow neutron resonance absorption peaks, is placed in the scattered beam, acting as a filter for the energy selection. In this latter case, data acquisition can be operated either in the Filter Difference Spectrometer (FDS) $[22,67,28,73]$ or in the Resonance Detector Spectrometer (RDS) [15,74,75,18,16,76,77] configurations (see below). A list of suitable materials to be used as analyser foils is shown in Table 2. This method for energy analysis is commonly used on inverse geometry instruments, since better resolution and count rates can be obtained in this geometry [31,78]. The main advantage of method (3) is that inelastic neutron scattering measurements can be performed in an unlimited range of neutron energy transfer (energy loss) as shown in Fig. 9, where iso-angular loci are plotted as a function of the energy and wave vector transfer for a given final neutron energy.

As an example, if one considers an inverse geometry spectrometer with $L_{0}=11.055 \mathrm{~m}, L_{1}=0.5 \mathrm{~m}$, and $E_{1}=4.908 \mathrm{eV}$, a typical fixed-angle time of flight spectrum is recorded in the range $50 \mu \mathrm{s} \leq t \leq 500 \mu \mathrm{s}$ with constant time bins of $0.5 \mu \mathrm{s}$, i.e. 900 time channels. We stress that this corresponds, for each detector element, to 900 different values of $\hbar \omega$ and $q$ [79]. Figs. 10-12 report the accessed $\hbar \omega, q$ values for this particular choice of $L_{0}, L_{1}$ and $E_{1}$. as a function of the neutron TOF.

In order to further extend the kinematic range, the option of longer flight paths can be considered. Novel generation spallation neutron sources can provide two orders of magnitude increase in the count rate for electron volt spectrometers. For example the choice of longer primary flight path $L_{0}=22.00 \mathrm{~m}$, that can be easily accomplished at SNS or J-PARC [80], would further extend the high limit values of $\hbar \omega, q$, as shown in Fig. 13.

For the specific case of proton recoil scattering studies, the crossing of the kinematic range with the proton recoil line is reported in Fig. 14.

The energy, $\Delta \hbar \omega$, and wave vector, $\Delta q$, resolution can both be written as a sum of different contributions associated with the uncertainties in the geometrical parameters $\left(L_{0} ; L_{1} ; \vartheta\right)$, the time of flight $t$ and the selected final energy $E_{1}$. Analytical estimates for approximating the energy and wave vector resolution can be carried out by considering $q$ and $\hbar \omega$ as a function of the (experimental) variables

$$
\begin{aligned}
& \hbar \omega=\hbar \omega\left(t, E_{1}, L_{0}, L_{1}\right) \\
& q=q\left(t, E_{1}, L_{0}, L_{1}, \vartheta\right) .
\end{aligned}
$$

Denoting the uncertainties on these variables by $\Delta t, \Delta E_{1}, \Delta L_{0}, \Delta L_{1}, \Delta \vartheta$, and assuming that they are independent of each other $[81,82,66,83,84,76]$, Gaussian distributed and sufficiently narrow for the differentials to be considered constant over their range, we obtain:

$$
(\Delta \hbar \omega)^{2}=\left[1+\left(\frac{E_{0}}{E_{1}}\right)^{3 / 2} \frac{L_{1}}{L_{0}}\right]^{2}\left(\Delta E_{1}\right)^{2}+\left[2 \frac{E_{0}^{3 / 2}}{A L_{0}}\right]^{2}(\Delta t)^{2}+\left[2 \frac{E_{0}^{3 / 2}}{L_{0} E_{1}^{1 / 2}}\right]^{2}\left(\Delta L_{1}\right)^{2}+\left[2 \frac{E_{0}}{L_{0}}\right]^{2}\left(\Delta L_{0}\right)^{2}
$$




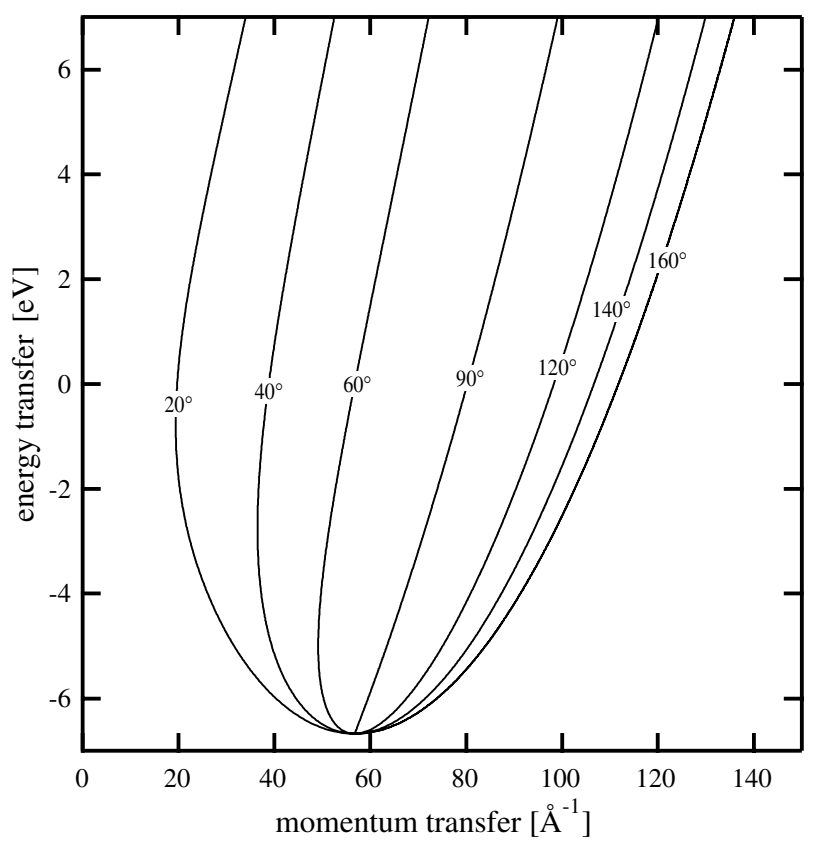

Fig. 9. Iso-angular loci of momentum and energy transfers for the inverse geometry configuration with $E_{1}=6.671 \mathrm{eV}$.

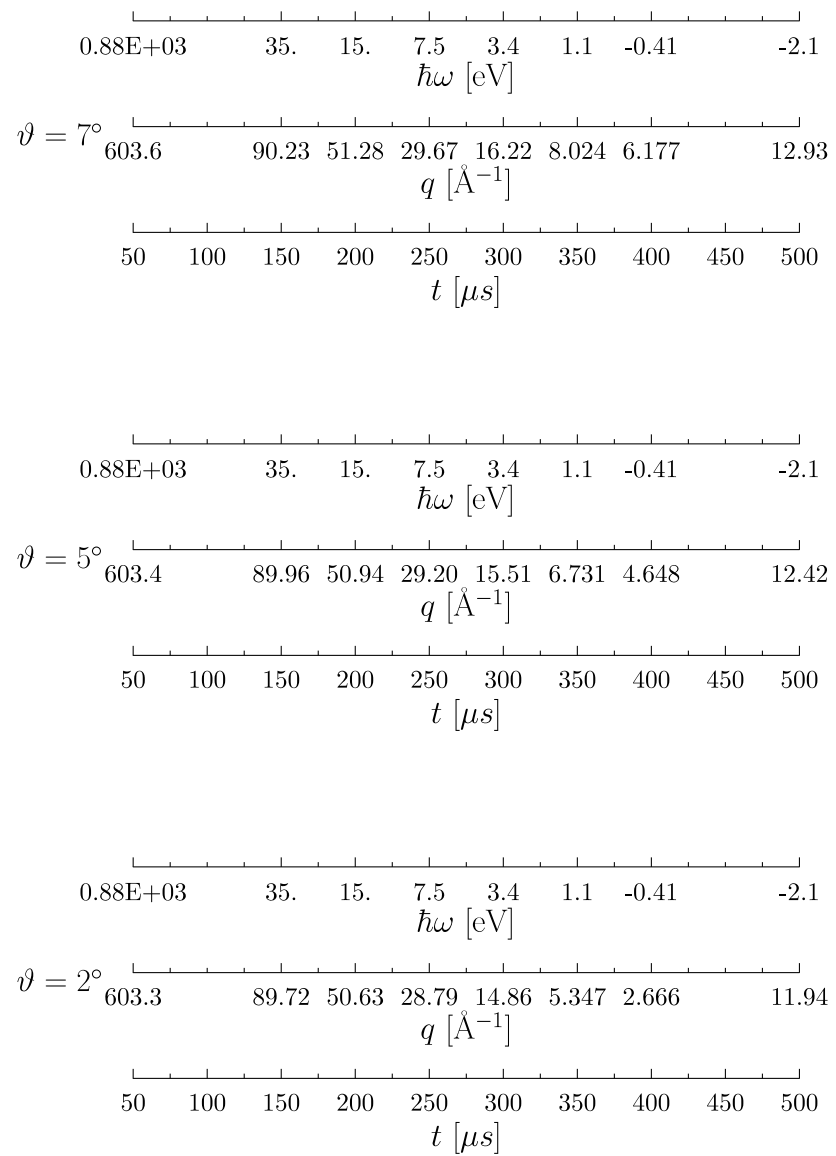

Fig. 10. Values of energy, $\hbar \omega$, and wave vector, $q$, transfers, for $L_{0}=11.055 \mathrm{~m}, L_{1}=0.5 \mathrm{~m}$, and $E_{0}=4.908 \mathrm{eV}$, as a function of the neutron time of flight for detection angles $\vartheta=2^{\circ}, 5^{\circ}, 7^{\circ}$. 


\begin{tabular}{|c|c|c|c|c|c|c|c|c|c|}
\hline \multirow{3}{*}{$\vartheta=70^{\circ}$} & $.88 \mathrm{E}+$ & & 35. & 15. & $\begin{array}{lr}7.5 & 3.4 \\
\hbar \omega & {[\mathrm{eV}]}\end{array}$ & 1.1 & -0.41 & & -2.1 \\
\hline & 636.9 & & 130.0 & 94.42 & $\begin{array}{c}76.03{ }^{65.41} \\
q\left[\AA^{-1}\right]\end{array}$ & 58.87 & 54.67 & & 49.99 \\
\hline & 50 & 100 & 150 & 200 & $\begin{array}{cc}250 & 300 \\
t & {[\mu s]}\end{array}$ & 350 & 400 & 450 & 500 \\
\hline
\end{tabular}

\begin{tabular}{|c|c|c|c|c|c|c|c|c|c|}
\hline & $.88 \mathrm{E}+0$ & & 35. & 15. & $\begin{array}{lr}7.5 & 3.4 \\
\hbar \omega & {[\mathrm{eV}]}\end{array}$ & 1.1 & -0.41 & & -2.1 \\
\hline \multirow[t]{2}{*}{$\vartheta=50^{\circ}$} & 621.7 & & 113.4 & 77.52 & $\begin{array}{c}59.2949 .22 \\
q\left[\AA^{-1}\right]\end{array}$ & 43.51 & 40.30 & & 37.69 \\
\hline & 50 & 100 & 150 & 200 & $\begin{array}{c}250 \quad 300 \\
t[\mu s]\end{array}$ & 350 & 400 & 450 & 500 \\
\hline
\end{tabular}

\begin{tabular}{|c|c|c|c|c|c|c|c|c|c|}
\hline \multirow{3}{*}{$\vartheta=30^{\circ}$} & $.88 \mathrm{E}+0$ & & 35. & 15. & $\begin{array}{lr}7.5 & 3.4 \\
\hbar \omega & {[\mathrm{eV}]}\end{array}$ & 1.1 & -0.41 & & -2.1 \\
\hline & 610.2 & & 99.22 & 62.07 & $\begin{array}{c}42.8232 .31 \\
q\left[\AA^{-1}\right]\end{array}$ & $\frac{1}{26.94}$ & 24.74 & & 24.91 \\
\hline & 50 & 100 & 150 & 200 & $\begin{array}{cc}1 & 1 \\
250 & 300 \\
t & {[\mu s]}\end{array}$ & $\frac{1}{350}$ & $\frac{1}{400}$ & $\frac{1}{450}$ & 500 \\
\hline
\end{tabular}

Fig. 11. Values of energy, $\hbar \omega$, and wave vector, $q$, transfers, for $L_{0}=11.055 \mathrm{~m}, L_{1}=0.5 \mathrm{~m}$, and $E_{0}=4.908 \mathrm{eV}$, as a function of the neutron time of flight for detection angles $\vartheta=30^{\circ}, 50^{\circ}, 70^{\circ}$.

$$
\begin{aligned}
(\Delta q)^{2}= & \left(\frac{B^{2}}{2 q}\right)^{2}\left[1-\left(\frac{E_{0}}{E_{1}}\right)^{3 / 2} \frac{L_{1}}{L_{0}}-\cos \vartheta\left(\left(\frac{E_{0}}{E_{1}}\right)^{1 / 2}-\frac{L_{1} E_{0}}{L_{0} E_{1}}\right)\right]^{2}\left(\Delta E_{1}\right)^{2} \\
& +\left(\frac{B^{2}}{2 q}\right)^{2}\left[\left[2 \frac{E_{0}^{3 / 2}}{A L_{0}}\right]^{2}(\Delta t)^{2}+\left[2 \frac{E_{0}^{3 / 2}}{L_{0} E_{1}^{1 / 2}}\right]^{2}\left(\Delta L_{1}\right)^{2}+\left[2 \frac{E_{0}}{L_{0}}\right]^{2}\left(\Delta L_{0}\right)^{2}\right] \\
& \times\left[\left(\frac{E_{1}}{E_{0}} \cos \vartheta-1\right)^{1 / 2}\right]^{2}+\left(\frac{B^{2}}{2 q}\right)^{2}\left[2\left(E_{0} E_{1}\right)^{1 / 2} \sin \vartheta\right]^{2}(\Delta \vartheta)^{2}
\end{aligned}
$$

where $A=(m / 2)^{1 / 2}=72.3 \mathrm{eV}^{1 / 2} \mu \mathrm{s} \mathrm{m}{ }^{-1}$, and $B=\left(2 / \hbar^{2}\right)^{1 / 2}=21.97 \AA^{-1} \mathrm{eV}^{-1 / 2}$.

The main components of the energy transfer resolution are $\Delta E_{1}$ and the ratio $\frac{L_{1}}{L_{0}}$. Further refinement of the resolution may be achieved using the time-focusing method, as pointed out by Carpenter et al. [19]. Table 3 reports the full width at half maximum of the energy transfer resolutions for different choices of the analyser foil, and for $L_{0}=11 \mathrm{~m}$ and $L_{1}=2 \mathrm{~m}$, i.e. $\frac{L_{1}}{L_{0}}=0.18$.

In order to derive a full determination of the resolution line shape we have carried out Monte Carlo simulations of the spectrometer response in the limit of infinitely narrow excitations, using a modified version of the DINSMS code [85]. The latter code provides a time of flight neutron transport for scattering from model scattering kernels in the eV range, that include the main characteristics of the spectrometer: the transfer matrices of the energy analysers, the velocity-dependent emission time from the moderator, a Gaussian probability distribution for the geometrical parameters, and multiple scattering. Data have been recorded for a scattering angle $\vartheta=50^{\circ}$, a sample with a delta-like excitation at $\hbar \omega=5000$ meV, and ${ }^{197} \mathrm{Au}$ resonance filters, varying both primary and secondary flight paths. Fig. 15 shows how the resolution improves by increasing the primary flight paths, for non-dispersive excitations of the form $S(q, \omega) \propto \delta(\hbar \omega-5000 \mathrm{meV})$. In agreement with the above calculations, the reduction of the ratio $\frac{L_{1}}{L_{0}}$ improves the energy resolution. 


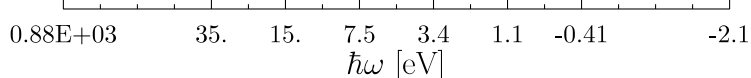

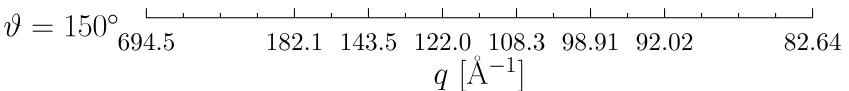

$$
\begin{aligned}
& \begin{array}{cccccccccc}
\leftarrow & 1 & 1 & 1 & 1 & 1 & 1 & 1 & 1 & 1 \\
50 & 100 & 150 & 200 & 250 & 300 & 350 & 400 & 450 & 500 \\
& & & & t[\mu s]
\end{array}
\end{aligned}
$$

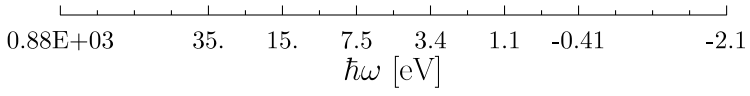

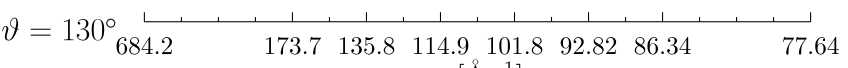

$$
\begin{aligned}
& q\left[\AA^{-1}\right]
\end{aligned}
$$

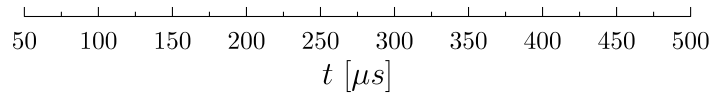

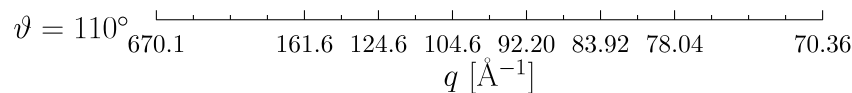

\begin{tabular}{|c|c|c|c|c|c|c|}
\hline $0.88 \mathrm{E}+03$ & 35. & 15. & $\begin{array}{lr}7.5 & 3.4 \\
\hbar \omega & {[\mathrm{eV}]}\end{array}$ & 1.1 & -0.41 & -2.1 \\
\hline
\end{tabular}

$$
\begin{aligned}
& \begin{array}{cccccccccc} 
& 1 & 1 & 1 & 1 & 1 & 1 & 1 & 1 & 1 \\
50 & 100 & 150 & 200 & 250 & 300 & 350 & 400 & 450 & 500 \\
& & & & t[\mu S]
\end{array}
\end{aligned}
$$

Fig. 12. Values of energy, $\hbar \omega$, and wave vector, $q$, transfers, for $L_{0}=11.055 \mathrm{~m}, L_{1}=0.5 \mathrm{~m}$, and $E_{0}=4.908 \mathrm{eV}$, as a function of the neutron time of flight for detection angles $\vartheta=110^{\circ}, 130^{\circ}, 150^{\circ}$.

\section{Table 3}

Resonance energy $E_{1}$ and FWHM, $\Delta E_{1}$ for the different foils at room temperature, taken as FWHM from the experimental neutron scattering cross section data available at [61]. $\Delta E_{1}$ represents the energy contribution to the instrument resolution. In the case of ${ }^{238} \mathrm{U}$ the value at a temperature $T=77 \mathrm{~K}$ is also reported, indicated with a $\left(^{*}\right)$. The columns 4-8 report the calculated resolution $\Delta \hbar \omega$ for selected values of the energy transfer $\hbar \omega$.

\begin{tabular}{|c|c|c|c|c|c|c|c|}
\hline \multirow[t]{2}{*}{ Filter } & \multirow[t]{2}{*}{$E_{1}(\mathrm{eV})$} & \multirow[t]{2}{*}{$\Delta E_{1}(\mathrm{meV})$} & \multicolumn{5}{|l|}{$\Delta \hbar \omega(\mathrm{meV})$} \\
\hline & & & $\hbar \omega=10 \mathrm{meV}$ & $\hbar \omega=500 \mathrm{meV}$ & $\hbar \omega=3 \mathrm{eV}$ & $\hbar \omega=7 \mathrm{eV}$ & $\hbar \omega=20 \mathrm{eV}$ \\
\hline${ }^{149} \mathrm{Sm}$ & 0.872 & 83 & 98 & 113 & 224 & 492 & 1848 \\
\hline${ }^{240} \mathrm{Pu}$ & 1.06 & 56 & 66 & 74 & 133 & 272 & 965 \\
\hline${ }^{185} \mathrm{Re}$ & 2.16 & 58 & 69 & 73 & 99 & 157 & 429 \\
\hline${ }^{242} \mathrm{Pu}$ & 2.67 & 71 & 85 & 89 & 114 & 167 & 416 \\
\hline${ }^{197} \mathrm{Au}$ & 4.91 & 182 & 216 & 221 & 252 & 313 & 581 \\
\hline${ }^{238} \mathrm{U}$ & 6.67 & $103\left(66^{*}\right)$ & 125 & 128 & 144 & 174 & 307 \\
\hline${ }^{187}$ Os & 12.7 & 100 & 135 & 138 & 151 & 177 & 286 \\
\hline${ }^{150} \mathrm{Sm}$ & 20.7 & 261 & 331 & 334 & 351 & 379 & 495 \\
\hline${ }^{238} \mathrm{U}$ & 20.9 & 177 & 243 & 246 & 262 & 290 & 404 \\
\hline${ }^{238} \mathrm{U}$ & 36.6 & 242 & 387 & 391 & 411 & 446 & 578 \\
\hline${ }^{238} \mathrm{U}$ & 66.0 & 320 & 701 & 707 & 736 & 784 & 952 \\
\hline${ }^{139} \mathrm{La}$ & 72.1 & 436 & 844 & 850 & 879 & 928 & 1098 \\
\hline${ }^{168} \mathrm{Er}$ & 79.7 & 120 & 785 & 791 & 826 & 883 & 1075 \\
\hline${ }^{238} \mathrm{U}$ & 102.6 & 410 & 1210 & 1217 & 1254 & 1315 & 1521 \\
\hline
\end{tabular}

However it has to be noted that for energy excitations of the form $\hbar \omega=\frac{\hbar^{2} q^{2}}{2 M}$, such as in the case of free recoil scattering in the deep inelastic neutron scattering (DINS) regime (briefly summarized later in this report) [41], the $q$-resolution is intimately connected to, and dominates, the energy resolution. From the above equations, it is possible to show that an increased secondary flight path reduces the energy as well as the angular components of the total energy resolution. To this aim, we have carried out Monte Carlo simulations of the spectrometer response to an infinitely narrow proton recoil signal, using the DINSMS code: data have been recorded for a scattering angle of $\vartheta=50^{\circ}$, a delta-like momentum width of 


\begin{tabular}{|c|c|c|c|c|c|c|c|c|c|}
\hline \multirow{3}{*}{$\vartheta=70^{\circ}$} & $.35 \mathrm{E}+0$ & & $0.15 \mathrm{E}+0$ & 376 & $\begin{array}{l}44 . \\
\hbar \omega[\mathrm{eV}]\end{array}$ & 19. & 13. & & 6.3 \\
\hline & 1285 & & 263.4 & 187.0 & $\begin{array}{c}145.1119 .0 \\
q\left[\AA^{-1}\right]\end{array}$ & 101.4 & 88.96 & & 72.93 \\
\hline & 50 & 100 & 150 & 200 & $\begin{array}{cc}250 & 300 \\
t[\mu s]\end{array}$ & 350 & 400 & 450 & 500 \\
\hline
\end{tabular}

\begin{tabular}{|c|c|c|c|c|c|c|c|c|c|}
\hline \multirow{3}{*}{$\vartheta=50^{\circ}$} & $.35 \mathrm{E}+04$ & & $0.15 \mathrm{E}+0$ & 376. & $\begin{array}{l}44 . \\
\hbar \omega[\mathrm{eV}]\end{array}$ & 19. & 13. & & 6.3 \\
\hline & 1270. & & 247.5 & $1 / 170.8$ & $\begin{array}{c}128.6102 .3 \\
q\left[\AA^{-1}\right]\end{array}$ & $\frac{1}{84.54}$ & $\frac{1}{72.06}$ & & 56.29 \\
\hline & 50 & 100 & 150 & 200 & $\begin{array}{cc}250 & 300 \\
t & {[\mu s]}\end{array}$ & 350 & 400 & 450 & 500 \\
\hline
\end{tabular}

\begin{tabular}{|c|c|c|c|c|c|c|c|c|c|}
\hline \multirow{3}{*}{$\vartheta=30^{\circ}$} & $.35 \mathrm{E}+04$ & & $0.15 \mathrm{E}+0$ & 376. & $\begin{array}{l}28 . \\
\hbar \omega[\mathrm{eV}]\end{array}$ & 19. & 13. & & 6.3 \\
\hline & 1259. & & 235.1 & 15 & $\begin{array}{c}114.887 .79 \\
q\left[\AA^{-1}\right]\end{array}$ & $\frac{1}{69.41}$ & $\frac{1}{56.32}$ & & 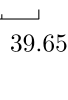 \\
\hline & 50 & 100 & 150 & 200 & $\begin{array}{c}250 \quad 300 \\
t[\mu s]\end{array}$ & 350 & 400 & 450 & 500 \\
\hline
\end{tabular}

Fig. 13. Values of energy, $\hbar \omega$, and wave vector, $q$, transfers, for $L_{0}=22.00 \mathrm{~m}, L_{1}=0.5 \mathrm{~m}$, and $E_{0}=4.908 \mathrm{eV}$, as a function of the neutron time of flight for detection angles $\vartheta=30^{\circ}, 50^{\circ}, 70^{\circ}$.

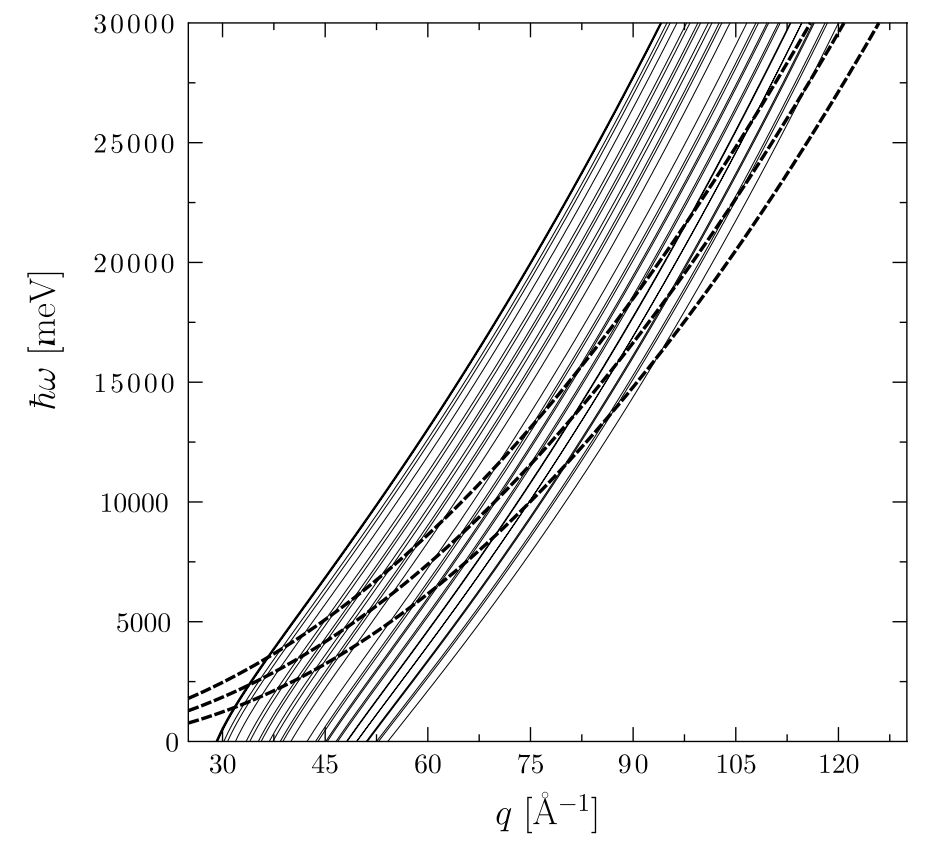

Fig. 14. Typical fixed-angle $q$, $\hbar \omega$ values accessed in proton momentum distribution measurements, for detectors in the angular range $32^{\circ} \leq \vartheta \leq 67^{\circ}$ (continuous lines); proton recoil line with a line width of $5 \AA^{-1}$ is also reported (dashed lines). 


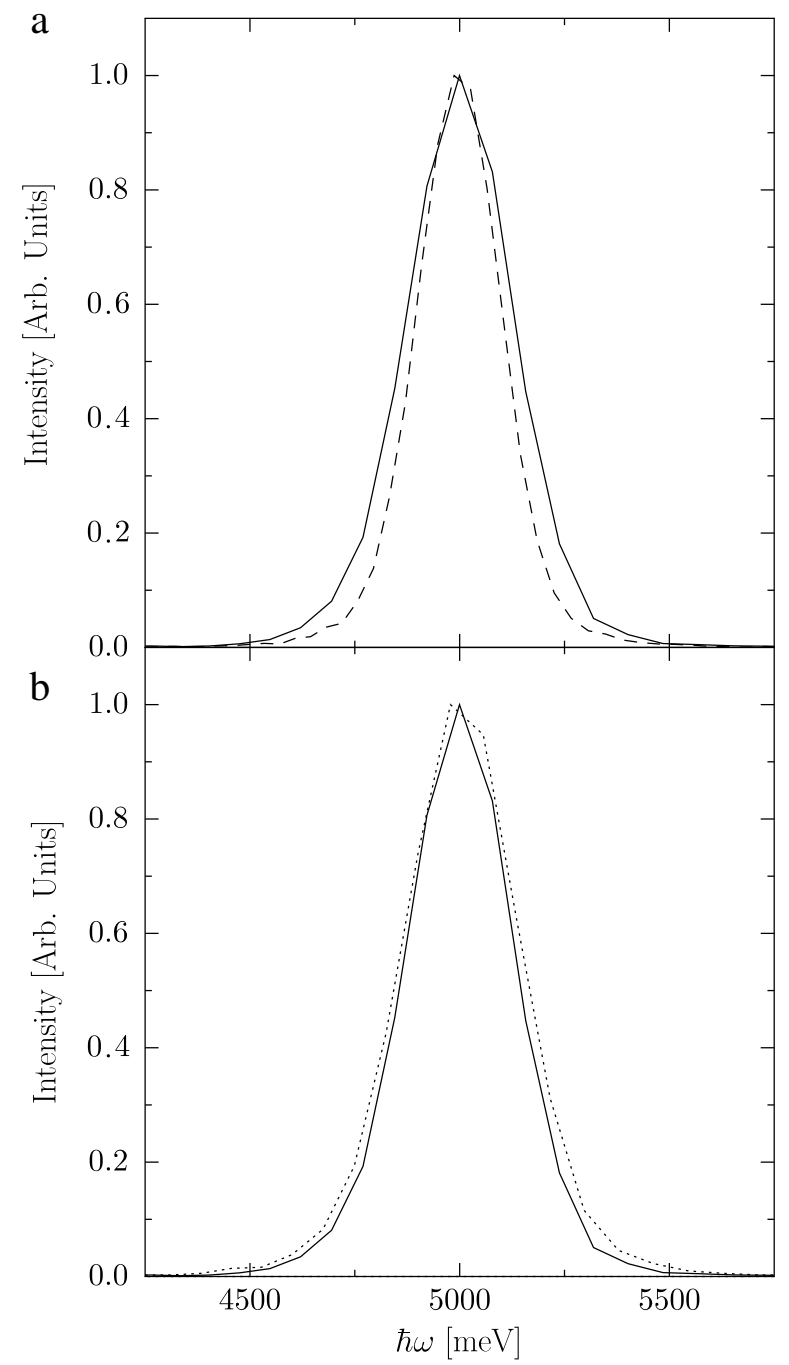

Fig. 15. Effect of flight paths contributions to the spectrometer resolution for high energy inelastic scattering experiments, with a non-dispersive excitation of the form $S(q, \omega) \propto \delta(\hbar \omega-5000 \mathrm{meV})$. The inelastic spectra were simulated using the DINSMS code for a scattering angle of $\vartheta=50^{\circ}$, a deltalike excitation at $\hbar \omega=5000 \mathrm{meV}$, and ${ }^{197} \mathrm{Au}$ resonance filters. (a) Effect of an increase in the primary flight path: the continuous line corresponds to $L_{0}=11.055 \mathrm{~m}, L_{1}=0.70 \mathrm{~m}$; the dashed line corresponds to $L_{0}=22.000 \mathrm{~m}, L_{1}=0.70 \mathrm{~m}$. (b) Effect of an increase in the secondary flight path: the continuous line corresponds to $L_{0}=11.055 \mathrm{~m}, L_{1}=0.70 \mathrm{~m}$; the dotted line corresponds to $L_{0}=11.055 \mathrm{~m}, L_{1}=1.40 \mathrm{~m}$.

$0.005 \AA^{-1}$, and ${ }^{197} \mathrm{Au}$ resonance filters, varying both primary and secondary flight paths. Fig. 16 shows the narrowing of the resolution by increasing flight paths, clearly showing that, for DINS measurements, the angular resolution dominates the wave vector, and, in turn, the energy resolution of the instrument.

It therefore appears that the requirements for optimizing the flight paths have to meet a compromise for simultaneous DINS and high energy inelastic neutron scattering at low angles (HINS) measurements. It is instructive to compare the resolution performances of direct and inverse geometry instruments for multi-eV scattering. Fig. 17 reports the raw time of flight spectrum of an hydrogen containing prespex sample, $5 \mathrm{~mm}$ thickness, recorded at a scattering angle $\vartheta=3.2^{\circ}$, using ${ }^{238} \mathrm{U}$ analyser [30]. The latter allows energy selections at multiple final energies, namely $6.67 \mathrm{eV}, 20.8 \mathrm{eV}, 36.2 \mathrm{eV}, 66.0$ $\mathrm{eV}$, etc. The time of flight spectrum is therefore composed of four regions at different time of flights, corresponding to the contributions of multiple energy selection. In particular, the proton elastic signals are visible for the final energies of 6.67, $20.8,36.6$ and $66.6 \mathrm{eV}$.

The spectrum can be transformed from TOF to four energy transfer representations, one for each final energy, i.e. the uranium resonance energy, to obtain the spectrum in different kinematic regimes. The filter difference technique can in principle be applied also in direct geometry configuration. The resonance energy of a filter placed in the incident flight path defines the initial neutron velocity, while the TOF technique allows the kinematics reconstruction of the scattering event. The energy width of the resonances, as shown for example in Table 3, determines the main resolution component, as in the indirect geometry case. The limitations in the kinematical space accessed, and on the possibility to optimize the flight 


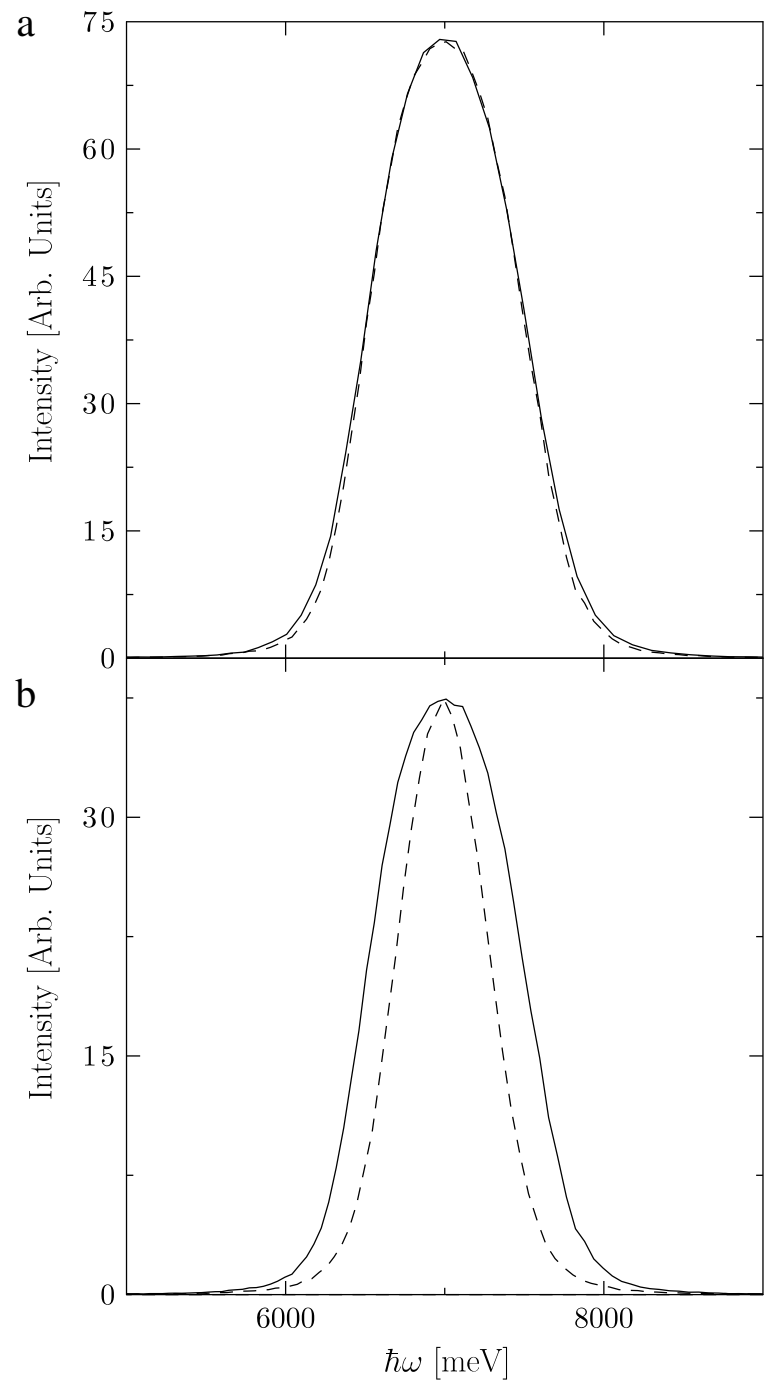

Fig. 16. Effect of flight paths contributions to the spectrometer resolution for DINS experiments, where $\hbar \omega \simeq \frac{\hbar^{2} q^{2}}{2 M}$. In the case of proton recoil scattering, $M=1.0079$ a.m.u., DINS spectra were simulated using the DINSMS code for a scattering angle of $\vartheta=50^{\circ}$, a delta-like momentum width of $0.005 \AA^{-1}$, and ${ }^{197} \mathrm{Au}$ resonance filters. (a) Effect of an increase in the primary flight path: the continuous line corresponds to $L_{0}=11.055 \mathrm{~m}, L_{1}=0.70 \mathrm{~m}$; dashed line corresponds to $L_{0}=22.000 \mathrm{~m}, L_{1}=0.70 \mathrm{~m}$. (b) Effect of an increase in the secondary flight path: the continuous line corresponds to $L_{0}=22.000 \mathrm{~m}$, $L_{1}=0.70 \mathrm{~m}$; dashed line corresponds to $L_{0}=22.000 \mathrm{~m}, L_{1}=1.40 \mathrm{~m}$.

Table 4

Resonance energy $E_{0}$ and FWHM, $\Delta E_{0}$ for the different foils at room temperature, taken as FWHM from the experimental neutron scattering cross section data available at [61]. $\Delta E_{0}$ represents the energy contribution to the instrument resolution. The columns 4-6 report the calculated resolution $\Delta \hbar \omega$ for selected values of the energy transfer $\hbar \omega$ for an eV spectrometer in direct geometry using ${ }^{238} \mathrm{U}$ filter for two particular resonance energies.

\begin{tabular}{lclllc}
\hline Filter & $E_{0}(\mathrm{eV})$ & $\Delta E_{0}(\mathrm{meV})$ & \multicolumn{2}{l}{$\Delta \hbar \omega(\mathrm{meV})$} & \\
\cline { 4 - 6 } & & & $\hbar \omega=10 \mathrm{meV}$ & $\hbar \omega=500 \mathrm{meV}$ & $\hbar \omega=3 \mathrm{eV}$ \\
\hline${ }^{238} \mathrm{U}$ & 6.67 & 103 & 696 & 633 & 346 \\
${ }^{238} \mathrm{U}$ & 36.6 & 242 & 1290 & 1268 & 1159 \\
\hline
\end{tabular}

paths ratio $\frac{L_{1}}{L_{0}}$, determined a scarce use of direct geometry instrument for scattering in the multi-eV range [67,27]. In the pioneering study of Ref. [67] the filter difference was applied to both direct and inverse geometry case. As an example, we report below in Table 4 the calculated spectrometer resolution $\Delta \hbar \omega$ for selected values of the energy transfer $\hbar \omega$, similarly to the case of Table 3 above. 


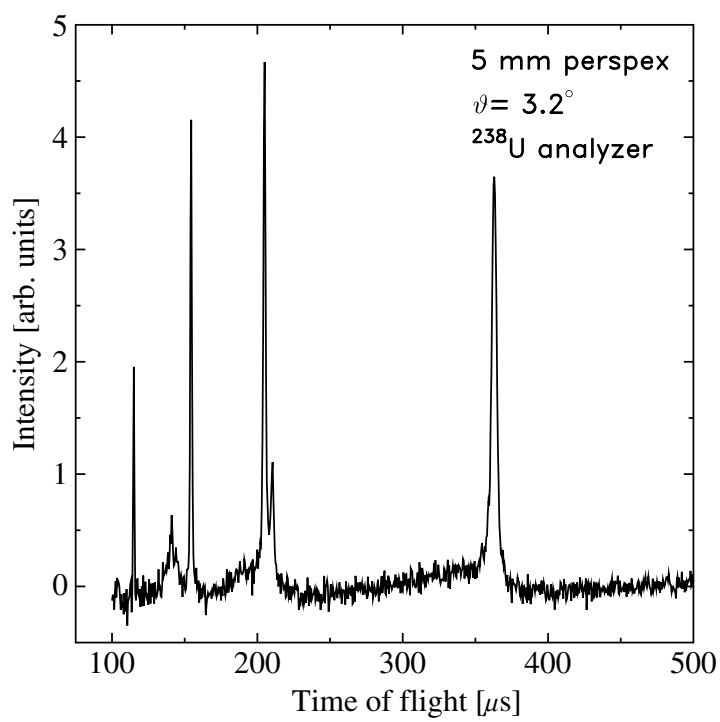

Fig. 17. Raw time of flight spectrum of the hydrogen containing prespex sample, $5 \mathrm{~mm}$ thickness, recorded at a scattering angle of $\vartheta=3.2^{\circ}$, employing ${ }^{238} \mathrm{U}$ analyser [30].

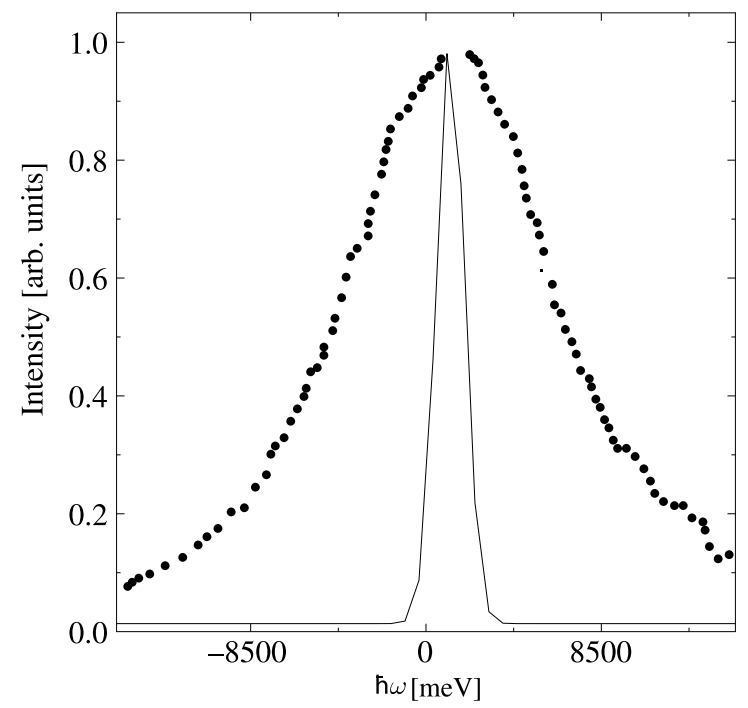

Fig. 18. Energy-transfer spectrum for scattering from hydrogen recorded on the MARI chopper spectrometer with incident energy $E_{0}=40000 \mathrm{meV}$, for scattering angle $\vartheta=5^{\circ}$ [27] (dots); calculated spectrum for a direct geometry resonance filter spectrometer using $E_{0}=36670$ meV (continuous line, see Table 4).

The above table shows that the spectrometer resolution using resonance filters in both configuration are of the same order of magnitude. Indeed, in direct geometry, the most common velocity selector device is the mechanical chopper [37]; however experimental data that allow for a precise characterization of the energy resolution of chopper instruments for incident energies above $5 \mathrm{eV}$ are not available so far [27]. Nevertheless it is possible to compare the calculated resolution for resonance filter direct geometry and chopper direct geometry in specific cases. Fig. 18 shows the low angle scattering from hydrogen, derived from experimental data with a direct geometry chopper spectrometer with an incident energy of $40 \mathrm{eV}$ and scattering angle $\vartheta=5^{\circ}$. In this kinematical regime, the broadening of the scattering spectrum is strongly dominated by the instrumental resolution. As a comparison, in the same figure we report the calculated spectrum for a direct geometry resonance filter spectrometer using the $36.67 \mathrm{eV}$ incident energy given by the third uranium resonance. The figure illustrates that for direct geometry with resonance filter, the energy resolution is approximately ten times smaller than in the chopper configuration.

In order to allow a more quantitative comparison between direct and inverse geometry performances, Fig. 19 reports the inverse-geometry spectra in the region of the elastic peak, where $E_{0} \simeq E_{1}$, obtained at three final neutron energies [30], together with a spectrum obtained in similar kinematical region, i.e. $E_{0}=40000 \mathrm{meV}$ and $\vartheta=5^{\circ}$, for a sample of low density polyethylene (LDPE) on the direct geometry chopper spectrometer MARI [27]. 

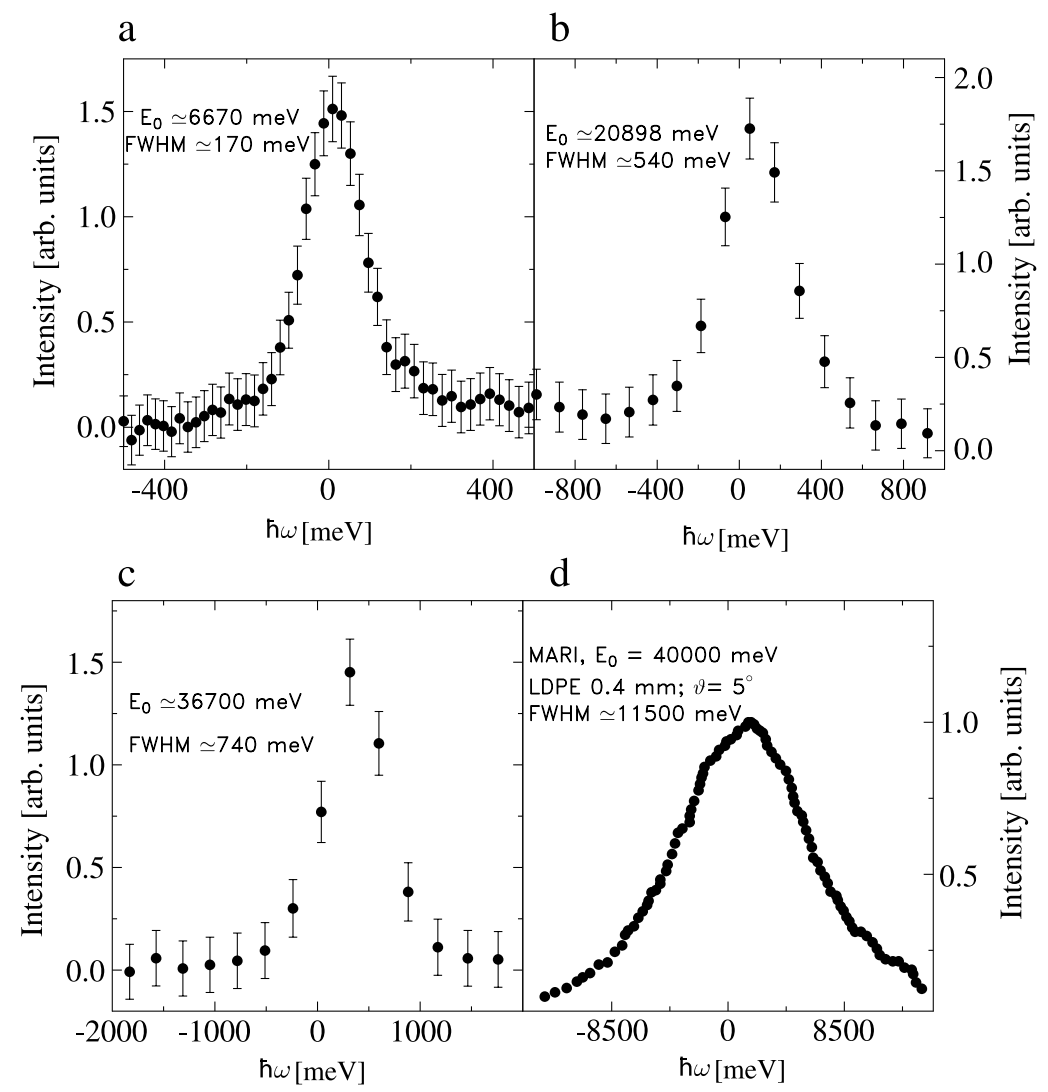

Fig. 19. Energy-transfer spectrum of the hydrogen containing prespex sample, $5 \mathrm{~mm}$ thickness, recorded at a scattering angle of $\vartheta=3.2^{\circ}$, using ${ }^{238} \mathrm{U}$ analyser, in the region of the elastic peak, for three final (initial) energies [30]: (a) $E_{1}=6671 \mathrm{meV}$; (b) $E_{1}=20898 \mathrm{meV}$; (c) $E_{1}=36700 \mathrm{meV}$; (d) elastic hydrogen peak recorded on the MARI chopper spectrometer with incident energy $E_{0}=40000 \mathrm{meV}$ for scattering angle $\vartheta=5^{\circ}$ [27].

The latter figure provides the energy width at the elastic peak for low angle scattering using incident (final) energies above $5 \mathrm{eV}$. It is instructive to compare the performances of direct and inverse geometry spectrometers in this kinematic range. From the observed Full width at Half Maximum (FWHM) of the elastic peak it is shown that, at the current state of the art, the resolution on inverse geometry resonance spectrometer is narrower by a factor between 15 and 60 with respect to direct geometry choppers [67]. In order to assess the expected performance of an inverse geometry eV spectrometer at low scattering angles, it is possible to consider the energy spectrum associated to the intramolecular $\mathrm{O}-\mathrm{H}$ stretching excitation in polycrystalline ice Ih. The neutron energy spectrum can be characterized as composed of a region of an elastic peak, whose width is mainly determined by the spectrometer resolution, and an inelastic peak located at $\hbar \omega \simeq 420 \mathrm{meV}$ corresponding to the $\mathrm{O}-\mathrm{H}$ stretching excitation [86]. A simulation of the spectrum, in energy transfer representation, for a scattering angle $\vartheta=2.0^{\circ}$, with a ${ }^{238} \mathrm{U}$ analyser at $E_{1}=6671 \mathrm{meV}$ is reported in Fig. 20 .

The simulated spectrum reproduces to a good approximation the experimental spectrum in similar configuration, as measured in 2004 [87,88], and a comparison of the experimental and simulated spectra is reported in Fig. 21.

Another case of interest for $\mathrm{eV}$ neutron scattering at low angles is the measurement of intermultiplet transitions in rare earths [89]. The typical splitting of electronic levels of rare earth systems lie in the hundreds of meV-eV range. For example, intermultiplet transitions were observed in Pr metal using the direct geometry HET spectrometer at ISIS [90,91], up to $810 \mathrm{meV}$. The measurement allowed to identify both dipolar $\left({ }^{3} \mathrm{H}_{4} \rightarrow{ }^{3} \mathrm{H}_{5}\right)$ and, for the first time, nondipolar, interterm $\left({ }^{3} \mathrm{H}_{4} \rightarrow{ }^{3} \mathrm{~F}_{2,3,4}\right)$ transitions. Such experiments are sensitive to the spin-orbit and Coulomb interactions giving rise to multiplet splittings. The neutron cross-section was measured for incident energies up to $E_{0}=1300 \mathrm{meV}$, at low scattering angles, below $\vartheta=5.0^{\circ}$, and with a wave vector transfer $q<10 \AA^{-1}$. This is a peculiar advantage of inelastic neutron scattering, in that the wave vector dependence of such excitations can be determined, with special regards to optically opaque materials. For this type of experiment with a direct geometry chopper the energy resolution is a factor about two narrower than for an inverse eV spectrometer. It is however illustrative to simulate a similar measurement with an inverse geometry instrument, thanks to the possibility to attain lower $q$-values at a given energy transfer. A time of flight spectrum was simulated for metallic Pr, using a model scattering function of the form:

$$
\frac{d^{2} \sigma}{d \Omega d E_{1}}=\frac{k_{1}}{k_{0}} \sigma_{n} S_{n}(\mathbf{q}, \omega)+\frac{k_{1}}{k_{0}} r_{0}^{2} G(\mathbf{q} ; \mu, v) \delta\left(\hbar \omega+E_{\mu}-E_{v}\right)
$$




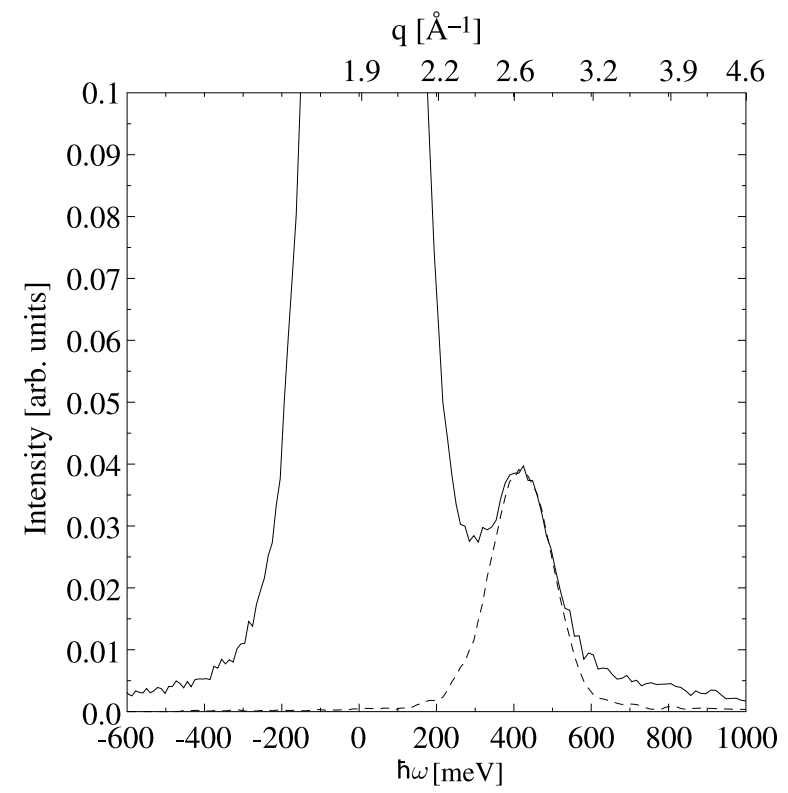

Fig. 20. Monte Carlo simulation of an energy-transfer spectrum of polycrystalline Ice Ih, recorded at a scattering angle of $\vartheta=2.0^{\circ}$, employing a ${ }^{238} \mathrm{U}$ analyser at $E_{1}=6671 \mathrm{meV}$ (continuous line); the spectrometer resolution at the $\mathrm{O}-\mathrm{H}$ excitation energy is also reported as a dashed line. The wave vector transfer values are reported in the top axis.
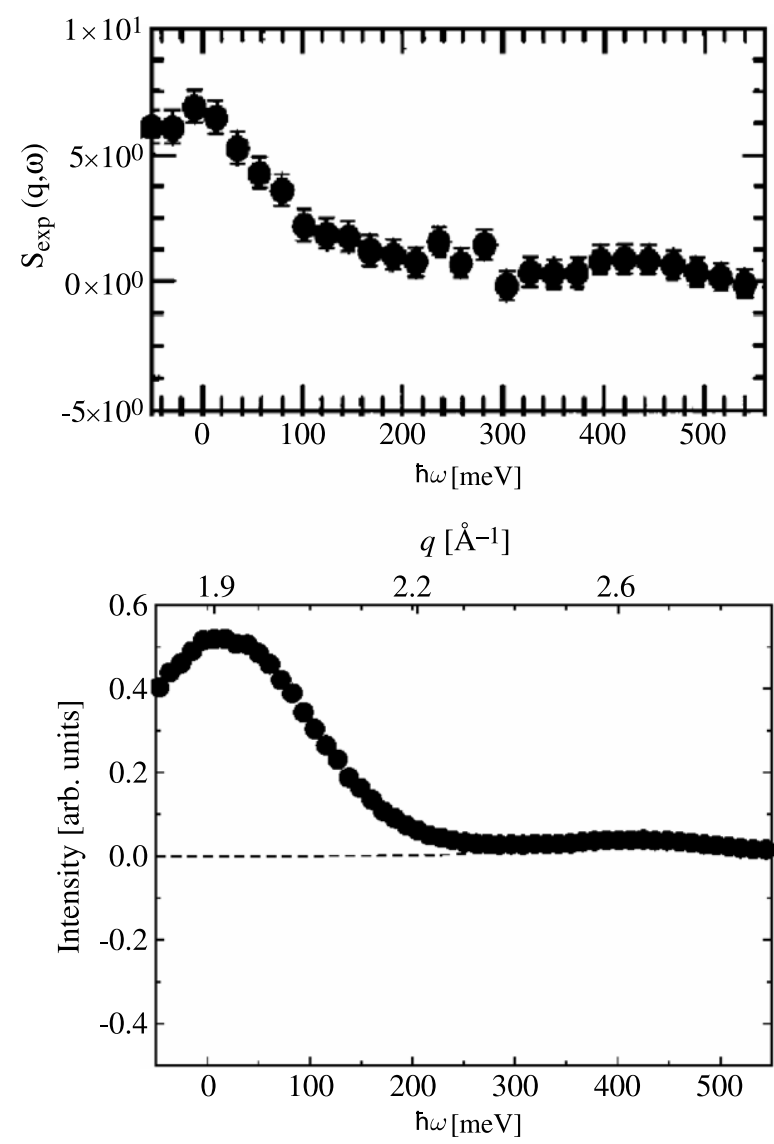

Fig. 21. Experimental (upper panel) and Monte Carlo-simulated (lower panel) energy-transfer spectrum of polycrystalline Ice Ih (same as in Fig. 20), recorded at a scattering angle of $\vartheta=2.0^{\circ}$, employing a ${ }^{238} \mathrm{U}$ analyser at $E_{1}=6671 \mathrm{meV}$ (circles with error bars). Source: Top panel from Ref. [87]. 


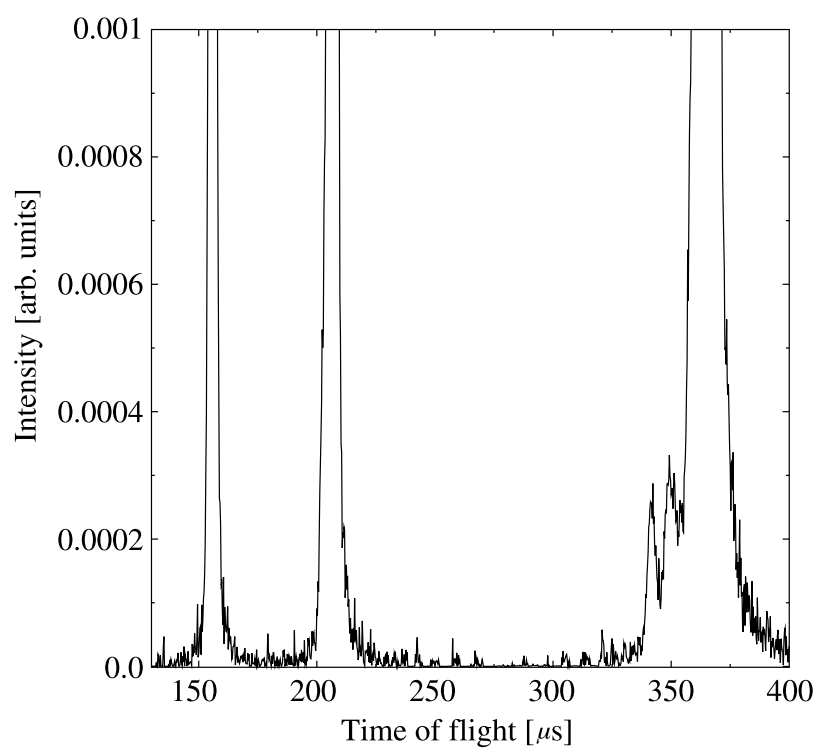

Fig. 22. Monte Carlo simulation of scattering from metallic praseodymium, recorded at a scattering angle of $\vartheta=2.0^{\circ}$, employing a ${ }^{238} \mathrm{U}$ analyser.

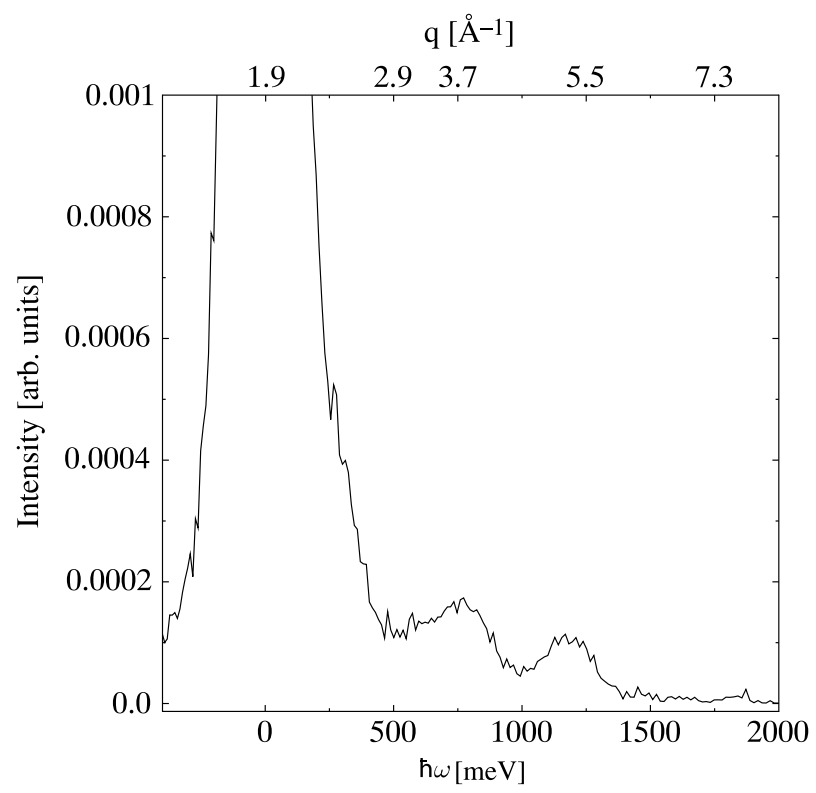

Fig. 23. Energy transfer spectrum of a Monte Carlo simulation of scattering from metallic praseodymium, recorded at a scattering angle of $\vartheta=2.0^{\circ}$, employing a ${ }^{238} \mathrm{U}$ analyser with $E_{1}=6671 \mathrm{meV}$. The wave vector transfer values are reported on the top axis.

where $S_{n}$ is the nuclear term associated to nuclear scattering, $r_{0}^{2}=0.29$ barn, $G(\mathbf{q} ; \mu, \nu)$ is the structure factor associated to transitions between states labelled by quantum numbers $\mu, v$; the excitation energies considered were: $261 \mathrm{meV}, 578 \mathrm{meV}$, $747 \mathrm{meV}, 809 \mathrm{meV}$, and $1170 \mathrm{meV}$, respectively [90]. Fig. 22 reports the simulated time of flight spectrum employing uranium analysers and a scattering angle of $\vartheta=2.0^{\circ}$.

Fig. 23 reports the corresponding energy transfer spectrum, employing the first uranium resonance at $E_{1}=6671 \mathrm{meV}$. Due to the broader energy resolution, with respect to a chopper spectrometer in this energy range, the excitation at $261 \mathrm{meV}$ is only visible as a shoulder on the nuclear elastic signal, while higher energy excitations are visible, with a lower wave vector transfer with respect to a chopper instrument.

\subsection{Filter Difference Spectrometers}

A first example of inverse geometry electron Volt spectrometer is the so-called Filter Difference Spectrometer (FDS) $[28,67,22]$. The FDS relies on the use of a scattered neutron energy selection system made of a filter analyser, such as one of 


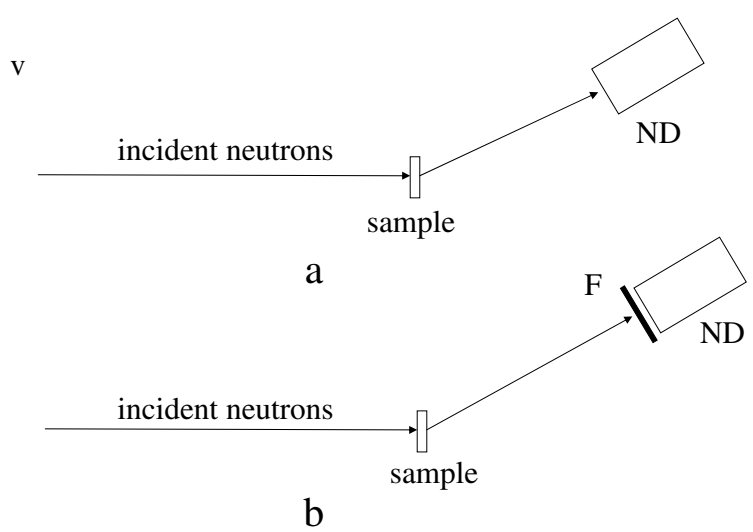

Fig. 24. Principles of operation of a Filter Difference Spectrometer (FDS): $\mathrm{F}$ is the resonant filter and ND is the neutron detector.

the materials listed in Table 2, and a neutron counter. The latter is typically a neutron sensitive scintillator, e.g. several mm thick Li-glasses, or a ${ }^{3} \mathrm{He}$-based gas counters at pressures of about $10 \mathrm{~atm}$ in order to avoid, in both cases, the large efficiency losses due to the velocity dependence of the absorption probability. The FDS scattering signal is then reconstructed by subtracting the spectrum recorded with the analyser in front of the counter from the one recorded without it (see Fig. 24).

The experimental signal recorded by a FDS is a time of flight spectrum, representing the number of counts collected in a time channel of width $\delta t$ centred in $t$. The count rate per time bin is given, with a good degree of approximation, by the expression [92]:

$$
C(t,\langle\vartheta\rangle)=\beta \cdot I_{P} \int_{0}^{\infty} d E_{0} \Phi\left(E_{0}\right) \int_{0}^{\infty} d E_{1}\left(1-T\left(E_{1}\right)\right) \delta(\tau)\left(\frac{d^{2} \sigma}{d \Omega d \hbar \omega}\right),
$$

where $\beta$ is given by:

$$
\beta=n_{T} \Delta \Omega \rho_{s} d_{s} \eta,
$$

$I_{P}$ is defined as:

$$
I_{P}=\int_{-\infty}^{\infty} d t_{0} \int_{0}^{\pi} d \vartheta \int_{0}^{\infty} d L_{0} \int_{0}^{\infty} d L_{1} P\left(t_{0}, \vartheta, L_{0}, L_{1}\right)
$$

and the argument in the $\delta$-function is:

$$
\tau=\left(t-t_{0}-L_{0} \sqrt{\frac{m_{n}}{2 E_{0}}}-L_{1} \sqrt{\frac{m_{n}}{2 E_{1}}}\right) .
$$

In the previous set of Eqs. (9)-(12), $m_{n}$ is the neutron mass, $n_{T}$ is the number of neutron pulses included in the measurement, $\Delta \Omega$ is solid angle defining the detector acceptance, while $\rho_{s}$ and $d_{s}$ are the sample density and thickness, respectively; $P\left(t_{0}, \vartheta, L_{0}, L_{1}\right)$ is the probability distribution that a given neutron leaves the moderator with a time-delay $t_{0}$, travels from the moderator to the sample along a flight path $L_{0}$, is scattered at an angle $\vartheta$, and finally travels from the sample to the detector along the secondary flight path $L_{1}$.

The quantities $\Phi\left(E_{0}\right), T\left(E_{1}\right), \frac{d^{2} \sigma}{d \Omega d \hbar \omega}$ and $\eta$ are the neutron flux at the incident neutron energy, the energy transfer function of the analyser and the double differential scattering cross section of the sample and the overall efficiency, respectively.

The energy-dependent transmission function of the filter is given by [93-95]:

$$
T(E)=1-e^{-\rho \sigma_{e f f} x \bar{f}(E)}
$$

where $\bar{f}(E)=\frac{f(E)}{f\left(E_{0}\right)}$ is the normalized nuclear absorption profile, $\sigma_{\text {eff }}$ the effective absorption cross-section at the peak of the resonance, $x$ the thickness and $\rho$ the nuclear density of the filter, respectively. The line shape of the cross section in the resonance region can be well approximated by:

$$
L(E)=\frac{\Gamma_{0}}{\pi\left[\Gamma_{0}^{2}+\left(E-E_{r}\right)^{2}\right]} .
$$

$E_{r}$ being the resonance energy and $\Gamma_{0}$, independent of $E$, the half-width at half-maximum (HWHM). As far as the Gaussian line shape (accounting for the Doppler broadening) is concerned, it can be shown that its standard deviation, $\sigma_{T}$, is proportional to the square root of the analyser effective temperature, $T^{*}$; times the neutron energy $E$, i.e. $\sigma_{T}=\left(\frac{2 m_{n} E T^{*}}{M_{a}}\right)^{0.5}, M_{a}$ being the 


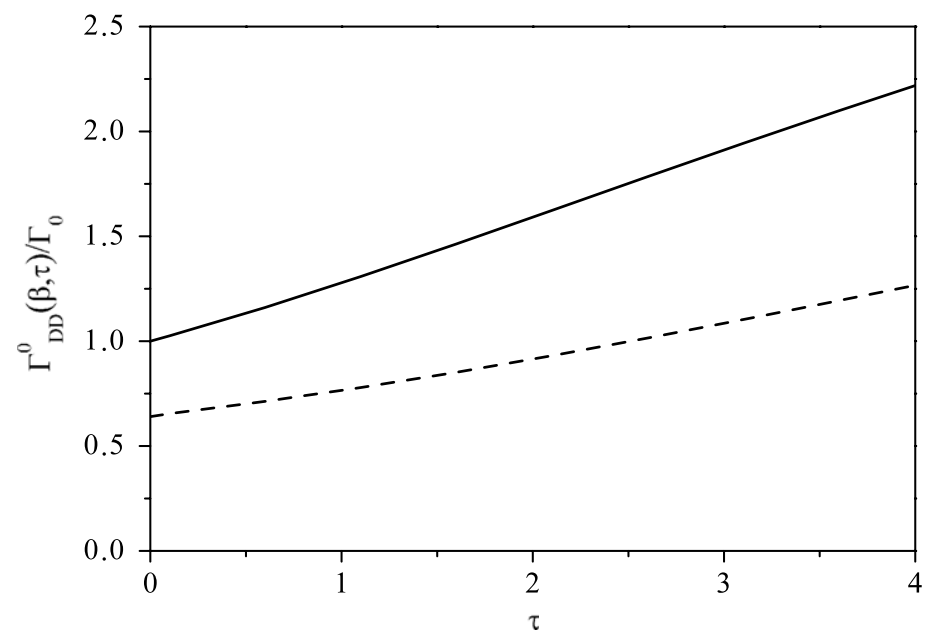

Fig. 25. Ratio between the half width at half maximum (HWHM) of the DDM transfer function in the zero temperature limit, $\Gamma_{D D M}^{0}(\beta, \tau)$ and the Breit-Wigner HWHM, $\Gamma^{0}$ for both $\beta=0$ (full line) and $\beta=1$ (dashed line).

nuclear mass of the filter analyser, and $T^{*}$ is approximately related to the Debye temperature of the filter, $\Theta_{D}$ and to its temperature, $T$, through the formula

$$
T^{*}=\frac{3}{8} \Theta_{D} \operatorname{coth}\left(\frac{3 \Theta_{D}}{8 T}\right) \text {. }
$$

In a typical scattering experiment, the energy interval of interest is much less than $1 \mathrm{eV}$ around the resonance energy $E_{r}$ $\left(E_{r}=4.908 \mathrm{eV}\right.$ for ${ }^{197} \mathrm{Au}$ and $6.671 \mathrm{eV}$ for $\left.{ }^{238} \mathrm{U}\right)$. Within this energy interval, the $\sigma_{T}$ dependence on the energy can be neglected. Therefore, the Doppler broadened resonance line shape is given by a Voigt profile that reads:

$$
f(E)=\frac{1}{\sqrt{2 \pi \sigma_{T}^{2}}} \operatorname{Re}\left\{\operatorname{erfc}\left(\frac{\Gamma_{0}-i E+i E_{r}}{\sqrt{\left(2 \sigma_{T}^{2}\right)}}\right) \times\left(\frac{\left(\Gamma_{0}-i E+i E_{r}\right)^{2}}{2 \sigma_{T}^{2}}\right)\right\}
$$

where $f\left(E_{0}\right)=\frac{1}{\sqrt{\left(2 \pi \sigma_{T}^{2}\right)}} \operatorname{erfc}\left(\frac{\Gamma 0}{\sqrt{\left(2 \sigma_{T}^{2}\right)}}\right) e^{\Gamma_{0}^{2} / 2 \sigma_{T}^{2}}=\frac{\psi_{0}}{\pi \Gamma_{0}}$ In Eq. (16) the coefficient $\psi_{0}$ defines the ratio of Voigt profile to the corresponding Lorentzian profile peak at $E=E_{r}$. When $\sigma_{T} \rightarrow 0$ (i.e. in the limit $T^{*} \rightarrow 0$ ), then $\psi_{0}=1$; the line shape reduces to a simple Lorentzian with $\sigma_{\text {eff }}=\sigma_{0}$, that is the peak absorption cross-section of a bound nucleus.

Defining the dimensionless parameter $\tau=\rho \sigma_{e f f} x$, Eq. (13) can be rewritten as $T(E)=e^{-\tau \bar{f}(E)}$ and then the transfer function of the FDS can be expressed as

$$
X_{F D S}(E)=1-T(E) \text {. }
$$

A different method to record the scattering signal in a FDS is the so-called Double Difference Method (DDM) [94,95]. The DDM relies on the use of two filters of thickness $x_{1}$ and $x_{2}$, with $x_{2}=x_{1} / \beta(0 \leq \beta \leq 1)$, or at different temperatures [96], and the instrument transfer function is the result of the following linear combination (see Ref. [94,95]):

$$
X_{D D M}=\left[1-e^{-\tau \bar{f}(E)}\right]-\beta\left[1-e^{-\tau \bar{f}(E) / \beta}\right] .
$$

For $\beta=0$ one obtains the standard $X_{F D S}$, while in the opposite limit of $\beta=1, X_{D D M}$ vanishes identically. The HWHM for the transfer function $X_{D D M}$, namely $\Gamma_{D D M}(\beta, \tau)$, can be calculated analytically only for $\beta=0$ and in the zero effective temperature limit $T^{*}=0$ yielding

$$
\lim _{T^{*} \rightarrow 0} \Gamma_{D D M}(0, \tau)=\Gamma_{F D S}^{0}(\tau)=\Gamma_{0}\left\{\frac{\tau}{\ln \left[2 /\left(1+e^{-\tau}\right)\right]}-1\right\}^{0.5}
$$

where the superscript 0 indicates that the Doppler broadening is absent. The growth of $\Gamma_{\mathrm{FDS}}^{0}(\tau)$ with $\tau$ is shown in Fig. 25 .

Values of $\Gamma_{\mathrm{FDS}}(\tau)$ for non-zero effective temperatures have to be evaluated numerically, starting from Eq. (17). When $\beta \neq 0$ the HWHM of the transfer function $X_{D D M}(E)$ must be calculated numerically, even in the limit of $T^{*}=0$. In the limiting case of very large $\beta$ (namely $\rightarrow 1$ ), expanding Eq. (18) around $\beta=1$, one obtains:

$$
X_{D D M}(E) \simeq(1-\beta)\left[1-e^{-\tau \bar{f}(E)}\right]-\tau \bar{f}(E) e^{-\tau \bar{f}(E)} .
$$

This transfer function, that vanishes for $\beta=1$, has therefore a limiting line shape which is independent of $\beta$. Its HWHM is, in the zero effective-temperature limit, a smoothly varying function of $\tau$, that can be evaluated numerically, using the above Eq. (20). 


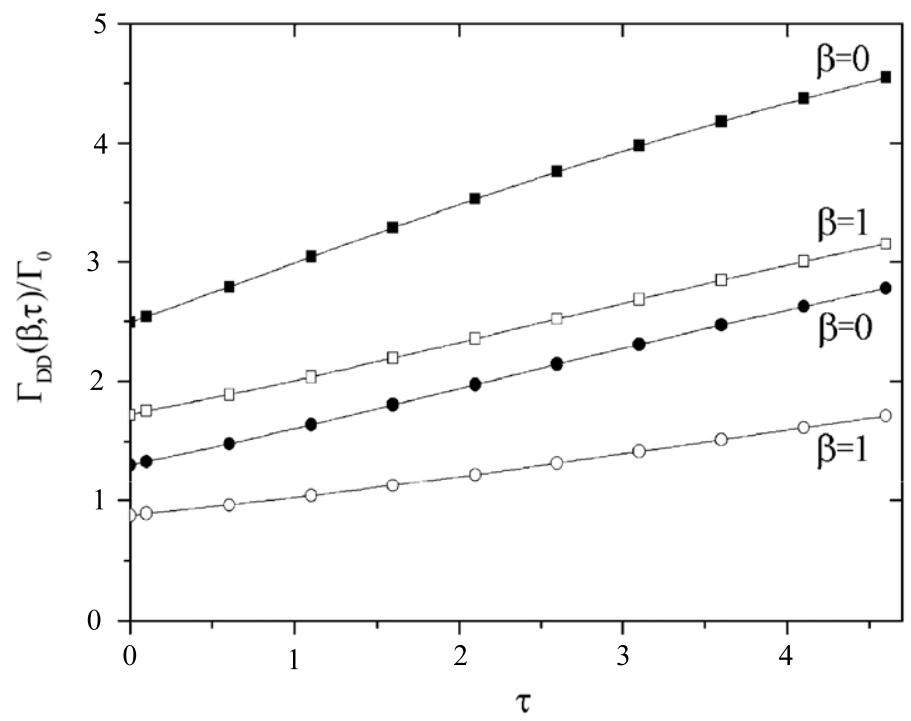

Fig. 26. Ratio between the HWHM $\Gamma_{D D M}^{0}(\beta, \tau)$ and $\Gamma_{0}$; calculated for a ${ }^{197}$ Au filter foil at room temperature (circles) and for ${ }^{238} \mathrm{U}$ at $T=77 \mathrm{~K}(\mathrm{squares}$ ), both for $\beta=0$ (full symbols) and $\beta=1$ (empty symbols). Lines are guides for the eye.

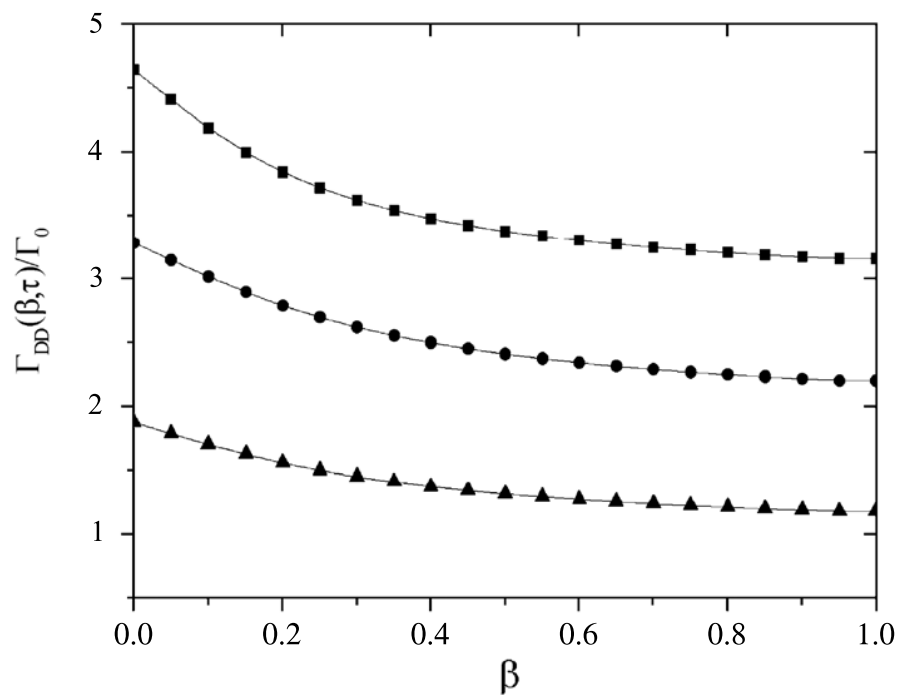

Fig. 27. Ratio between the HWHM $\Gamma_{D D M}(\beta, \tau)$ and $\Gamma_{0}$ as a function of $\beta$ for the ${ }^{238} \mathrm{U}$ foil (squares at room temperature and circles at liquid nitrogen temperature) and the ${ }^{197} \mathrm{Au}$ foil at room temperature (triangles) for the $\tau$ values of $1.1,1.8$, and 1.6 respectively. Lines are only a guide for eyes.

The ratio between $\Gamma_{D D M}^{0}(\beta \rightarrow 1, \tau) / \Gamma_{0}$ is reported as a function of $\tau$ in Fig. 25, for both $\beta=0$ and 1 . Intermediate $\beta$ values will yield values falling between these two curves for any given value $\tau$ of the thinner filter. It is worth noting that the value of $\Gamma_{D D M}^{0}(\beta=1, \tau)$ when $\tau=0$ represents, for a given value of $\Gamma_{0}$, the minimum attainable HWHM. This situation corresponds to the DDM performed using two identical, thin filters. The corresponding transfer function in the double limit $\tau \rightarrow 0, \beta \rightarrow 1$ can be worked out analytically expanding the exponential in Eq. (16) to obtain $X_{D D M}(E)=\frac{1}{2}(1-\beta) \tau^{2} \bar{f}(E)^{2}$, i.e. the square of a Lorentzian function, whose HWHM in the zero effective-temperature limit is $\Gamma_{0} \sqrt{2^{0.5}-1}=0.64 \Gamma_{0}$. In Fig. 26 are reported the numerical calculations of the ratio $\Gamma_{D D M}(\beta, \tau) / \Gamma_{0}$ for ${ }^{197} \mathrm{Au}$ and ${ }^{238} \mathrm{U}$ filters at room temperature, obtained in both limits $\beta=0$ and 1. In this figure, the $\beta=0$ limit corresponds to a FDS spectrum with a filter of thickness $\tau$, while the $\beta=1$ limit corresponds to a DDM spectrum obtained using two identical filters of thickness $\tau$.

All intermediate $\beta$ values yield curves laying between these two cases. An example of the dependence of $\Gamma_{D D M}(\tau)$ on $\beta$ is shown in Fig. 27.

The main advantages of DDM are: (1) a considerable improvement of the resolution and (2) a significant qualitative change in the shape of the Lorentzian wings of the transmission function. This occurs since the wings of $X_{F D S}(E)$ are independent of $\tau$, except for multiplication by $\tau$ (whenever $\bar{f}(E)$ is small, the expansion of the exponential yields $\bar{f}(E)$ itself). As a consequence, in the DDM configuration the wings of the transfer function are partially cancelled out with the 
Table 5

Main physical parameters of different nuclear resonances which can be employed in the RDS configuration: $E_{r}$ is the resonance energy, $\sigma_{0}$ the peak value of the resonance cross section and $\Gamma_{0}$ the half width at half maximum.

\begin{tabular}{lccc}
\hline Isotope & $E_{r}(\mathrm{eV})$ & $\sigma_{0}(\mathrm{~b})$ & $\Gamma_{0}(\mathrm{meV})$ \\
\hline${ }^{113} \mathrm{In}_{49}$ & 14.6 & 9965 & 67 \\
${ }^{139} \mathrm{La}_{57}$ & 72.2 & 5969 & 96 \\
${ }^{150} \mathrm{Sm}_{62}$ & 20.7 & 56207 & 109 \\
${ }^{160} \mathrm{Dy}_{66}$ & 20.5 & 16165 & 124 \\
${ }^{168} \mathrm{Er}_{68}$ & 79.7 & 11203 & 121 \\
${ }^{178} \mathrm{Hf}_{72}$ & 72.6 & 16838 & 112 \\
${ }^{182} \mathrm{~W}_{74}$ & 21.1 & 46800 & 104 \\
${ }^{190} \mathrm{Os}_{76}$ & 91.0 & 6777 & 105 \\
${ }^{197} \mathrm{Au}_{79}$ & 4.96 & 36592 & 60 \\
${ }^{238} \mathrm{U}_{92}$ & 6.67 & 23564 & 25 \\
${ }^{238} \mathrm{U}_{92}$ & 20.8 & 37966 & 34 \\
${ }^{238} \mathrm{U}_{92}$ & 36.6 & 42228 & 57 \\
${ }^{238} \mathrm{U}_{92}$ & 66.0 & 20134 & 48 \\
\hline
\end{tabular}

consequent improvement of the overall instrument resolution at the price of a reduced counting statistics. In the above discussion we gave only some of the main results that can be found in Ref. [94], to which the reader is referred to for a thorough discussion.

\subsection{The Resonance Detector Spectrometer}

A different class of inverse geometry electron Volt spectrometers is the so-called Resonance Detector Spectrometer(RDS). These consist of a scattered neutron energy selector made by a resonance analyser and a gamma detector. The RDS counting procedure relies upon two main steps $[97,31,98,99,76,100]$ : in the first one, the scattered neutron beam impinges onto the analyser foil which provides the energy analysis by means of $(n, \gamma)$ resonance absorption at a given resonance energy $E_{r}$; in the second step, the prompt gamma-rays are detected to assign the total neutron time of flight.

It has to be stressed that the gamma detector is used as a counter: for each absorbed neutron into the analyser, a gammaray cascade is produced with a certain probability. It is enough that one photon among the whole cascade is detected, even by a partial release of its energy in the detector (above a discrimination threshold that can be set electronically), to trigger a counting signal for the electronics. Also in the RDS configuration the scattering signal is reconstructed by performing the difference between analyser-in and analyser-out TOF spectra. The expression of the count rate for a RDS is similar to that shown in Eq. (9). It has to be stressed that the latter represents only the signal component, i.e. assuming the absence of background. A more complete expression should contain background terms, as it will be discussed in the following. One important advantage of using gamma detectors is that the wide photon spectrum from the analyser (atomic X-rays and nuclear gamma-rays) allows to use energy discrimination thresholds that improve the signal to background ratio, still guaranteeing a good counting efficiency [60].

\subsubsection{Choice of the neutron counting system in the RDS configuration}

The analyser foil has to fulfil some important requirements: (i) the radiative capture cross section has to show isolated and intense resonances in the energy region of interest $\left(E_{r}=1-100 \mathrm{eV}\right.$ ), (ii) these resonances should have small widths as compared to $E_{r}$, (iii) the emitted $\gamma$-ray spectrum should contain lines with appreciable relative intensities in a wide energy range. The first two requirements are important in order to properly select the scattered neutron energy and to ensure a low contribution to the energy component of the spectrometer's resolution function, respectively. The third requirement allows for selecting $\gamma$ energies to improve the $\mathrm{S} / \mathrm{B}$ ratio.

In Table 5, a number of suitable isotopes to be used in the RDS configuration are listed together with their main physical characteristics. According to the single level theory of Breit and Wigner [101] for an isolated nucleus, the neutron resonance absorption cross section is a Lorentzian function of the neutron energy in the laboratory frame where the nucleus is at rest (see Eq. (14)). In a real experiment the absorbing nucleus is embedded in a solid. In this case the resonance absorption profile departs from a pure Lorentzian due to the effect crystal lattice [102] and of the chemical bonding [103]. It can be shown that in the case of weak binding between absorbing atoms, defined by the condition [103]

$$
\Gamma_{0}+\sqrt{R k_{B} T_{e f f}} \gg k_{B} \Theta_{D},
$$

where $k_{B}$ is the Boltzmann's constant and $R$ is the recoil energy of the absorbing nucleus, the absorption cross section is given by

$$
\sigma=\sigma_{0} \cdot \Psi(\xi, x)
$$

with

$$
\Psi(\xi, x)=\frac{\xi}{2 \sqrt{\pi}} \int_{-\infty}^{\infty} d y \frac{\exp \left[-\left(\frac{\xi}{2}\right)^{2}\right](x-y)^{2}}{1+y^{2}}
$$




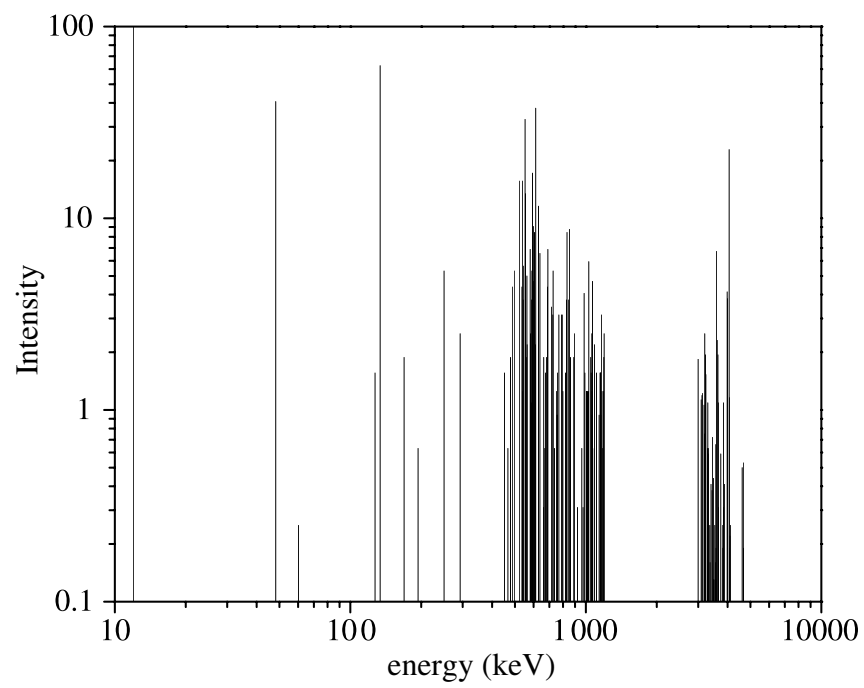

Fig. 28. Radiative capture gamma emission lines and intensities relative to most intense line at about $11 \mathrm{keV}$, for ${ }^{238} \mathrm{U}$ [104].

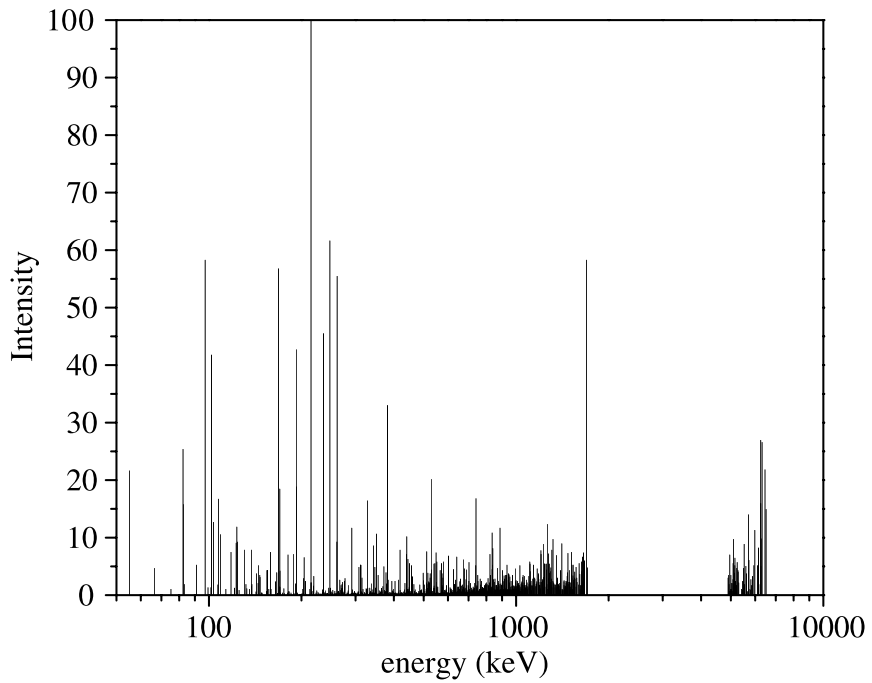

Fig. 29. Radiative capture gamma emission lines and intensities relative to the most intense line at about $215 \mathrm{keV}$, for ${ }^{197} \mathrm{Au}$ [105].

where $x=\frac{E-E_{r}-R}{\Gamma_{0}}$ and $\xi=\frac{\Gamma_{0}}{2 \sqrt{R k_{B} T_{e f f}}}$. In the weak binding approximation, the effect of the temperature and chemical bonding on the resonance profile is described by the effective energy spread:

$$
k_{B} T_{e f f}=\frac{1}{2} \int_{\infty}^{\infty} d E E g(E) \operatorname{coth}\left(\frac{E}{2 k_{B} T}\right),
$$

$T$ being the thermodynamic temperature and $g(E)$ the phonon density of states in the solid. The neutron absorption probability approaches 1 in the limit of very thick absorbers, however an increase in the absorber's thickness produces an increase of the resonance absorption profile width, thus worsening the resolution of a RDS and leading to possible selfshielding effects. An acceptable compromise between high absorption probability and energy resolution is given by the condition $N_{d} \sigma_{0}=1, N_{d}$ being the number density of resonant absorbing atoms per unit area perpendicular to the neutron beam.

Figs. 28 and 29 show histograms of the relative intensities as a function of the $\gamma$ line energy, for both ${ }^{197} \mathrm{Au}$ and ${ }^{238} \mathrm{U}$.

The role of the $\gamma$ detector in the RDS configuration is neutron counting. As described before, the resonance absorption of scattered neutrons into the analyser produces a prompt emission of $\gamma$-rays with a certain probability, whose detection is used to assign the neutron TOF by a proper signal processing electronics. The RDS counting rate depends on the neutron absorption probability in the analyser, the efficiency of the $\gamma$ detector and the discrimination threshold set in the measurements. 


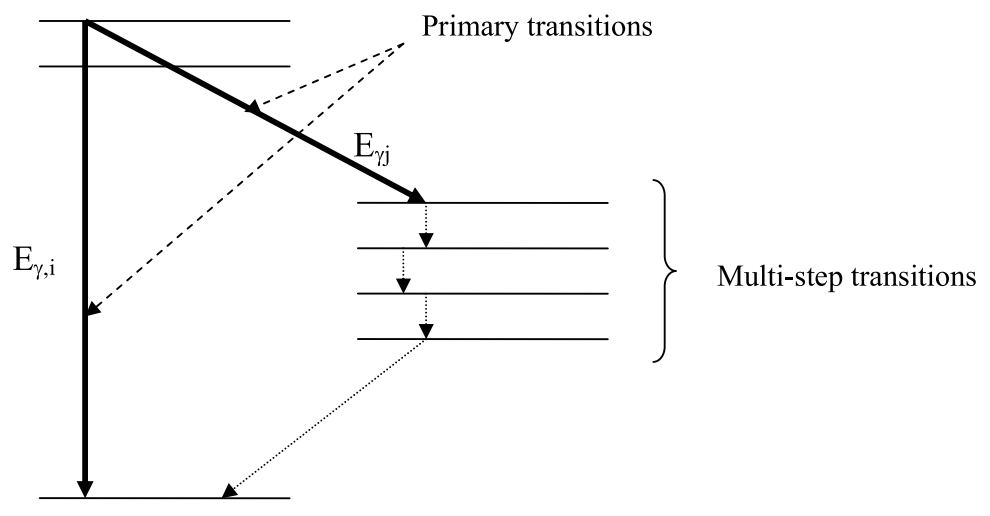

Fig. 30. Schematic picture describing primary and multi-step radiative transitions following resonance neutron capture.

In fact the environmental operation conditions of a spectrometer are characterized by the presence of heavy direct and neutron-induced photon background, and the use of discrimination thresholds is very useful for the optimization of the signal to background ratio $[59,58]$.

An important requirement for a $\gamma$ detector is the absence, in the active medium, of isotopes having neutron resonances in the 1-100 eV energy range. These resonances produce beam-correlated background featuring peaks that are superimposed to the main scattering peaks from the sample. Inorganic scintillators, commonly used for $\gamma$-ray spectroscopy, can be suitably employed in the RDS configuration because most of them fulfil the above mentioned requirements. The possibility to have crystals of different sizes allows to use them in different photon energy ranges. Despite these useful characteristics, radiation damage effects may be important and can cause reduction of transparency due to colour centres formation which absorb the scintillation light.

Solid state detectors such as high purity germanium or silicon are commonly used for energy resolved photon spectroscopy because of their very good energy resolution. For this class of semiconductors, radiation damage effects is a limiting factor for their routine use for the RDS configuration. Furthermore, due to a small band gap energy, these devices operate at low temperature in order to maintain a low leakage currents level. A different class of solid state detectors are the compound semiconductors such as Cadmium-Telluride (CdTe) and Cadmium-Zinc-Telluride (CZT) [106].

These materials can operate at room temperature thanks to larger band gap energies (in the order of $1-2 \mathrm{eV}$ ) as compared to Ge and Si. The larger mean atomic numbers for CdTe and CZT provide an higher photoelectric absorption probability and a higher efficiency for high energy $\gamma$-rays with respect to intrinsic Ge and Si semiconductors.

\subsubsection{Considerations on the detection efficiency for the RDS configuration}

The RDS counting efficiency is mostly independent of neutron energy, differently from what happens in the FDS configuration, as shown in a series of experimental works [60].

This feature can be explained on a physical ground, by referring to the simplified picture shown in Fig. 30 .

It is well known that the transitions probabilities, from states that are excited by the neutron capture, to different final states depend on the incoming neutron energy, the corresponding primary $\gamma$ emission width, $\Gamma_{\lambda f}$, following a Porter-Thomas distribution [107]. The secondary transitions, however, proceed from states that are fed by multi-step transitions from the capturing state, and are less dependent on neutron energy. Indeed, the spectrum of low energy transitions is largely independent of capturing states for target spin $I=0$ and $s$-wave neutrons. This argument is made plausible by considering that low energy lines represent depopulation of levels that are fed by many transitions; hence, their distribution is characterized by a variance $\frac{2}{v_{\text {eff }}}, v_{\text {eff }}$ being the effective number of contributing channels, which for a heavy nucleus such as ${ }^{238} \mathrm{U}$ is in the order of several hundreds. In such a case the line strength is closely proportional to the capture cross section. The appreciable fluctuations of the relative intensity with incoming neutron energy of the primary $\gamma$ transitions [108] do not appreciably affect the detection efficiency as the counting is provided by an integrated detection over a wide energy range, where secondary multi-step transitions are highly predominant. The efficiency $\eta_{d}$ can be affected by the self-absorption of the $\gamma$-rays within the analyser. This effect depends on the $\gamma$-ray energy and is negligible, as a first approximation, due to the small thickness of the foils (in the order of few tens of microns, depending on the resonance cross section) and to the high energy of the $\gamma$-rays (from few hundred of keV to MeV).

To estimate the detection efficiency $\eta_{d}$, let us consider a simplified situation, sketched in Fig. 31 .

In the figure, $n$ represents a scattered neutron of energy $E=E_{r}$ impinging normally to the analyser foil surface of thickness $t$. In a first order approximation (single neutron interaction) the neutron is absorbed after travelling a distance $x$ into the analyser. At the absorption point a single $\gamma$-ray of energy $E_{\gamma}$ is promptly emitted and may enter into the photon detector, that is placed very close to the analyser. In a very simple way it can be argued that the detection probability is given by $\eta_{d}=P_{n}\left(E_{r}\right) \cdot \bar{P}_{\gamma}\left(E_{\gamma}\right) \cdot I_{r}\left(E_{\gamma}\right) \cdot \varepsilon_{d}\left(E_{\gamma}\right)$, where $P_{n}\left(E_{r}\right)$ represents the neutron absorption probability in the analyser, $\bar{P}_{\gamma}\left(E_{\gamma}\right)$ 


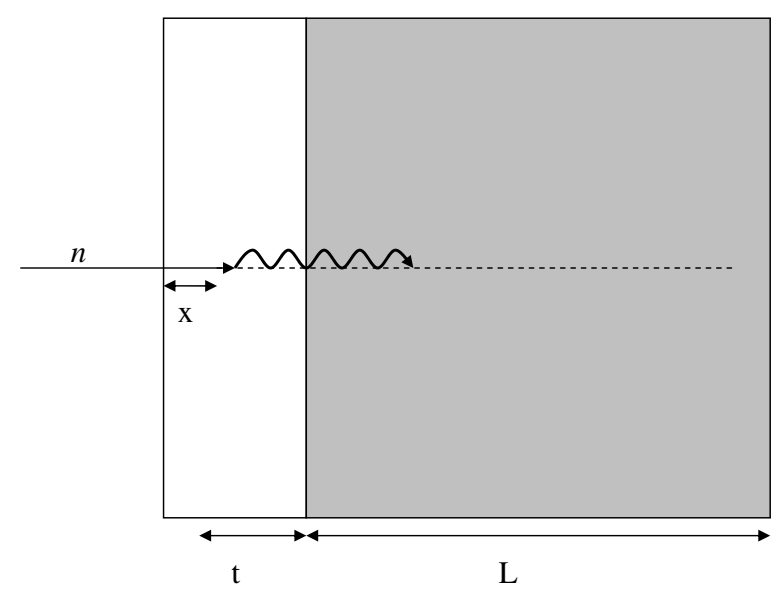

Fig. 31. Illustration describing the variables employed to evaluate the detection efficiency of a RD, in the simple case of normal incident neutrons onto the analyser and forward emission of photons towards the detector.

is the escape probability for the $\gamma$-ray, $I_{r}\left(E_{\gamma}\right)$ is the relative intensity of the $\gamma$ line and $\varepsilon_{d}\left(E_{\gamma}\right)$ is the detector efficiency for the line $E_{\gamma}$. The overall detection efficiency can be written, with the very simple assumption described above, as:

$$
\eta_{d}=I_{r}\left(E_{\gamma}\right) \cdot \varepsilon_{d}\left(E_{\gamma}\right) \cdot 0.5 \int_{0}^{t} \frac{1}{\lambda_{n}\left(E_{r}\right)} \cdot \exp \left[-\frac{x}{\lambda_{n}\left(E_{r}\right)}\right] \cdot \exp \left[-\frac{t-x}{\lambda_{\gamma}\left(E_{\gamma}\right)}\right] \cdot d x
$$

where integration over $x$ indicates that the escaping probability of the photon depends on the absorption point of the neutron, while the 0.5 factor takes into account the geometrical efficiency due to forward $\gamma$ emission. The result, after simple integration calculations, is:

$$
\eta_{d}=\frac{e^{-\frac{t}{\lambda_{\gamma}\left(E_{\gamma}\right)}}}{\lambda_{n}\left(E_{r}\right)} \cdot\left\{\frac{\bar{\beta}^{2}}{\widetilde{\lambda}} \cdot\left[e^{\frac{\tilde{\lambda}}{\beta^{2}}}-1\right]\right\} \cdot I_{r}\left(E_{\gamma}\right) \cdot \varepsilon_{d}\left(E_{\gamma}\right) .
$$

In Eq. (26) $\lambda_{\gamma}\left(E_{\gamma}\right)$ is the attenuation length for the $\gamma$ in the analyser, $\lambda_{n}\left(E_{r}\right)$ is the interaction length for the resonant neutron in the analyser, $\bar{\beta}^{2}=\lambda_{\gamma}\left(E_{\gamma}\right) \cdot \lambda_{n}\left(E_{r}\right)$ and $\tilde{\lambda}=\lambda_{\gamma}\left(E_{\gamma}\right)-\lambda_{n}\left(E_{r}\right)$. In order to make a numerical evaluation of the detection probability, let consider a neutron energy of $6.671 \mathrm{eV}$ and a ${ }^{238} \mathrm{U}$ analyser, assuming that a $138 \mathrm{keV} \gamma$-ray (see Fig. 28) is emitted. In this case the $\lambda_{n}\left(E_{r}\right) \simeq 30 \mu \mathrm{m}$, while $\lambda_{\gamma}\left(E_{\gamma}\right) \simeq 160 \mu \mathrm{m}$. Considering a relative intensity $I_{r}\left(E_{\gamma}\right)=0.65$ and a detector efficiency $\varepsilon_{d}\left(E_{\gamma}\right)=1$ a value $\eta_{d} \simeq 0.18$ is obtained. This calculation does not take into account the whole isotropic $\gamma$-ray cascade and the homogeneous distribution of scattered neutrons onto the analyser's surface, however gives an order of magnitude for $\eta_{d}$. Despite the very raw assumptions it can be argued that, due to the difference between $\lambda_{n}\left(E_{r}\right)$ and $\lambda_{\gamma}\left(E_{\gamma}\right)$, the self-absorption probability is not a dominant effect, being in the order of $12 \%$ for a $138 \mathrm{keV} \gamma$-ray in uranium.

By varying the neutron resonance energy, the radiative capture cross section also varies and thus the penetration depth of the neutron in the analyser. Anyway by properly tuning the analyser thickness to the cross section intensity, and considering that the $\gamma$ emission is mostly independent of neutron energy, a neutron energy independent $\eta_{d}$ is obtained.

\subsection{Hybrid configuration: the Foil Cycling Technique}

The width of the resolution function of a RDS can be narrowed by employing a signal recording method, henceforth called the Foil Cycling Technique (FCT), described schematically in Fig. 32.

It envisages the use of two foils of the same material: the first, the analyser foil, is placed in front of the photon detector and behaves as the standard energy analyser for the RDS configuration, while the second, the cycling foil, is placed between the sample and the analyser at a given distance from the latter. In the scattering experiment, the cycling foil alternates in and out of the scattered neutron beam, leaving the analyser in front of the photon detector. Subtracting the spectra acquired with and without the cycling foil produces the final data. The improvement of the resolution function is due to the narrowing of the FWHM of the transfer function using this technique. The FCT may be called a redundant resolution technique, one resolving method, the RDS, piled upon another, the FDS. The cycling foil acts as a filter and removes neutrons from the scattered beam having energies in the range of $\pm \Delta E_{r}$ around the resonance energy $E_{r}$. When the cycling foil is between the sample and the analyser, the photon detector registers the $\gamma$-rays produced by neutrons that pass the filter and are absorbed by the analyser. Photons produced by the cycling foil are much less effectively detected due to the smaller solid angle subtended by the detector, as the analyser-to-cycling foil distance is in the order of several tens of centimetres. The contribution of $\gamma$ produced by the cycling foil is further reduced by the subtraction procedure, as the number of $\gamma$-rays from the cycling foil registered by the detector can be made comparable for the foil-in and foil-out positions. 


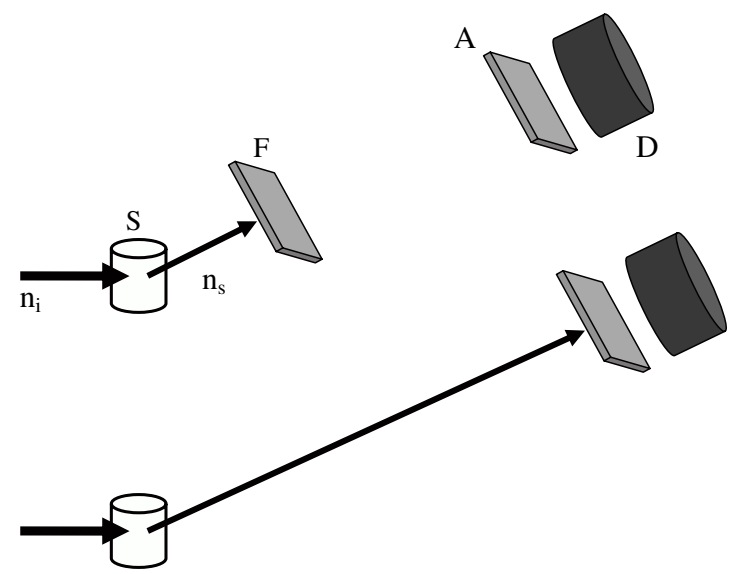

Fig. 32. Schematics of the principle of operation of the foil cycling technique.

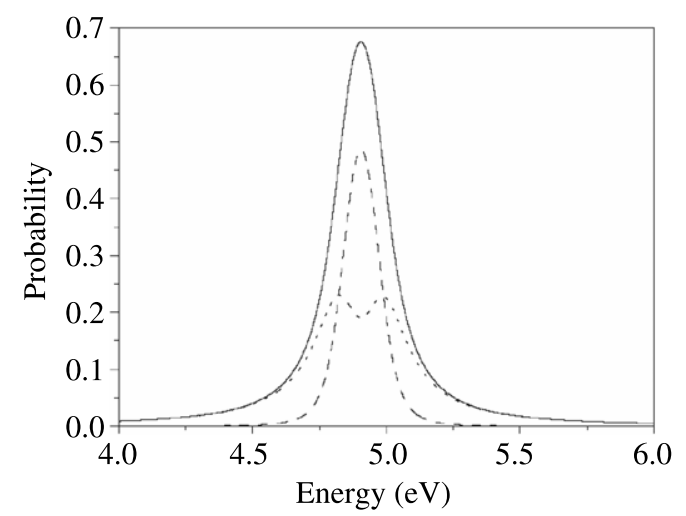

Fig. 33. Transfer functions for the single analyser foil (continuous line) and for the Foil Cycling Technique (dashed line). The dotted line represents the contribution of $T_{i} n(\mathrm{E})$.

The overall transfer function in this "hybrid configuration" of an $\mathrm{eV}$ spectrometer can be reconstructed considering the cross sections involved in the filtering procedure and can be written, accordingly to Eq. (13), as the difference between the cycling foil-out transfer function for an analyser foil of thickness $d_{1}$ and the cycling foil-in transfer function for a filter of thickness $d_{2}$ :

$$
T_{F}(E)=T_{\text {out }}(E)-T_{\text {in }}(E)=\left\{1-e^{\left[-N \sigma_{(n, \gamma)}(E) d_{1}\right]}\right\}-e^{\left[-N \sigma_{\text {tot }}(E) d_{2}\right]} \cdot\left\{1-e^{\left[-N \sigma_{(n, \gamma)}(E) d_{1}\right]}\right\} .
$$

Fig. 33 represents the transfer functions $T_{F C T}, T_{\text {out }}$ and $T_{i n}$, in the case of $d_{1}=d_{2}$, corresponding to a $7 \mu \mathrm{m}^{197} \mathrm{Au}$ analyser/filter. This thickness is about one interaction length for a neutron at the $4.906 \mathrm{eV}$ resonance energy of ${ }^{197} \mathrm{Au}$. The transfer function $T_{F}(E)$ has a FWHM which is appreciably narrower as compared to the standard RDS (i.e. $T_{\text {out }}(E)$ ), improving from about 236 to $160 \mathrm{meV}$. As far as the efficiency loss is concerned, the difference in the peak value of the interaction probability is about $30 \%$. The intensity reduction can be improved by properly tuning the thickness of the filter and the analyser, relaxing the resolution. Out of the resonance region the tails are depressed, even if not exactly cancelled. In fact the different cross sections involved $\sigma_{(n, \gamma)}(E)$ and $\sigma_{t o t}(E)$ have a different behaviour in the off-resonance regions, as it is well shown in Fig. 34.

At about $10 \mathrm{eV}$ the ratio of the two cross section is a factor of 4 , while at about $35 \mathrm{eV}$ is a factor of about 50 . The combination represented by Eq. (27) provides an overall transfer function characterized by lower tails, as compared to the standard RDS transfer function. The choice $d_{1}=d_{2}=d$ seems the most reliable in terms of counting efficiency and resolution improvement. As a matter of fact, fixing the analyser thickness at the value $d_{1}=\lambda$ and lowering the filter thickness $\left(d_{2}<\lambda\right)$, the $T_{i n}(E)$ functions would have higher tails and a less pronounced drop in correspondence of the resonance energy $E_{r}$. This provides a $T_{F}(E)$ characterized by a faster decrease of the tails, but an appreciable intensity reduction at the peak. On the contrary a thicker filter thickness $\left(d_{2}>\lambda\right)$ would provide a $T_{i n}(E)$ characterized by an appreciable drop at the resonance energy but would produce a $T_{F}(E)$ with a lower decrease in the tail region. The effect of a thicker analyser $\left(d_{1}>\lambda\right)$ would produce a gain in the peak intensity but an enlargement of the FWHM of $T_{\text {out }}(E)$ which would be less compensated in the difference procedure that provides $T_{F}(E)$.

A complete description of the resolution function line shape in the FCT configuration was done, and the reader is referred to Refs. $[110,111]$ for a thorough discussion. For sake of brevity, here below we report the main result of that investigation. 


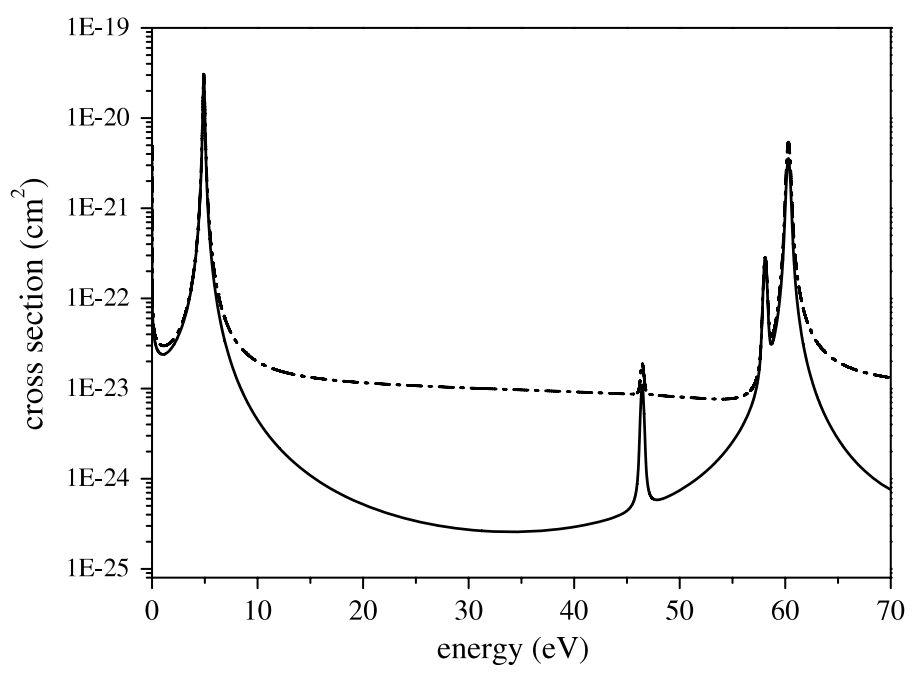

Fig. 34. Total (dashed-dotted line) and radiative capture (continuous line) cross-sections for ${ }^{197} \mathrm{Au}$ in the energy range up to $70 \mathrm{eV}$ [109].

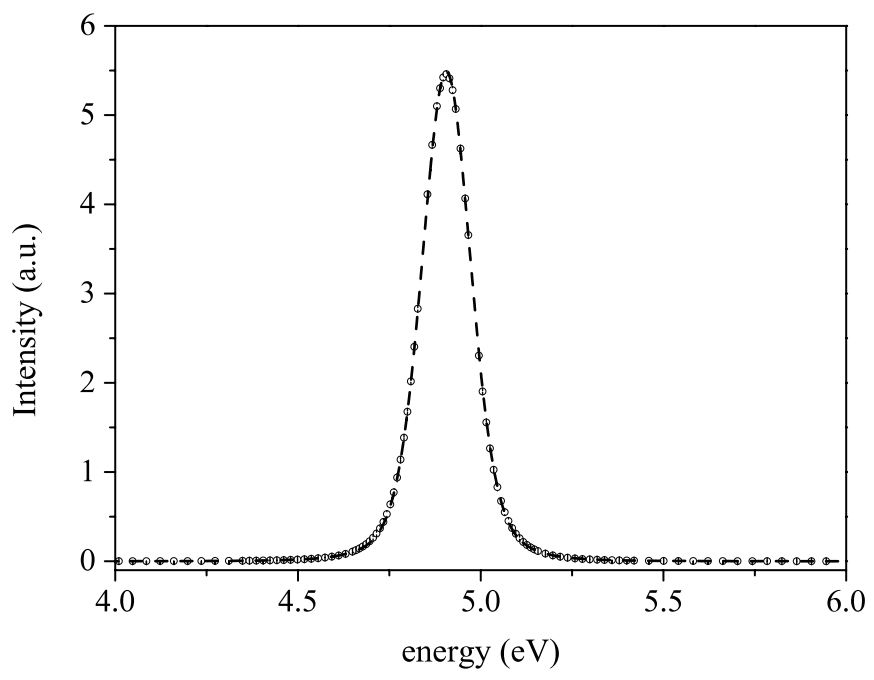

Fig. 35. Transfer function given by Eq. (27) (dots) and a fit (dashed line) obtained employing the form given by Eq. (28).

The functional form assumed for the FCT transfer function is given by a sum of a Gaussian and a power of a Lorentzian of the form:

$$
T_{G L}(E)=\frac{A_{G}}{\sqrt{2 \pi \sigma^{2}}} \cdot e^{-\frac{\left(E-E_{r}\right)^{2}}{2 \sigma^{2}}}+A_{L}\left\{\frac{\frac{\Gamma_{L}}{2}}{\pi\left[\left(E-E_{r}\right)^{2}-\frac{\Gamma_{L}^{2}}{4}\right]}\right\}^{\alpha},
$$

where $\sigma$ is the standard deviation of the normal energy distribution, $\Gamma_{L}$ is the FWHM of the Cauchy distribution and $A_{G}$ and $A_{L}$ are amplitude parameters ( $A_{L}$ with its dimensions).

Fig. 35 shows the transfer function built from Eq. (27) together with the best fit using Eq. (28).

A different set of fitting functions were also tested, but the choice of Eq. (28) is the most appropriate. This was also confirmed by a series of simulation of DINS spectra from a lead sample. For heavy mass atoms, such as Pb, the DINS line shape signal is dominated by the spectrometer resolution, so that this simulation was a very stringent test for the reliability of the transfer function. Indeed small deviations from the correct transfer function would result in appreciable differences between experimental and simulated spectra. In Fig. 36, the experimental and the simulated TOF spectra are reported in the region 250-500 $\mu \mathrm{s}$. The spectrum is relative to a YAP detector at the smallest angle in the array $\left(\vartheta \simeq 48^{\circ}\right)$. This has been chosen in order to maximize the contribution of the resolution (i.e. the geometrical component [81]) to the chosen transfer function $T_{F}(E)$. The simulation is in very good agreement with the experimental data with $\chi^{2}=1.03$. The average $\chi^{2}$, calculated over the whole set of the 64 detectors employed for the measurements, has a value $\left\langle\chi^{2}\right\rangle=1.04 \pm 0.06$. 


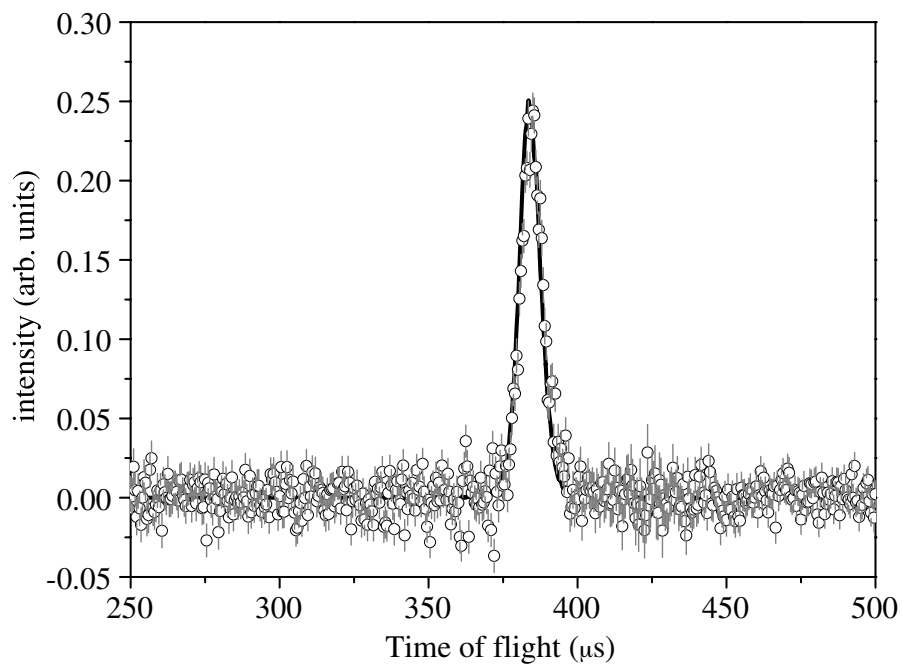

Fig. 36. Experimental (dots) and simulated recoil spectra (continuous line) from a lead sample, employing a transfer function based on Eq. (27), for a detector at about $48^{\circ}$.

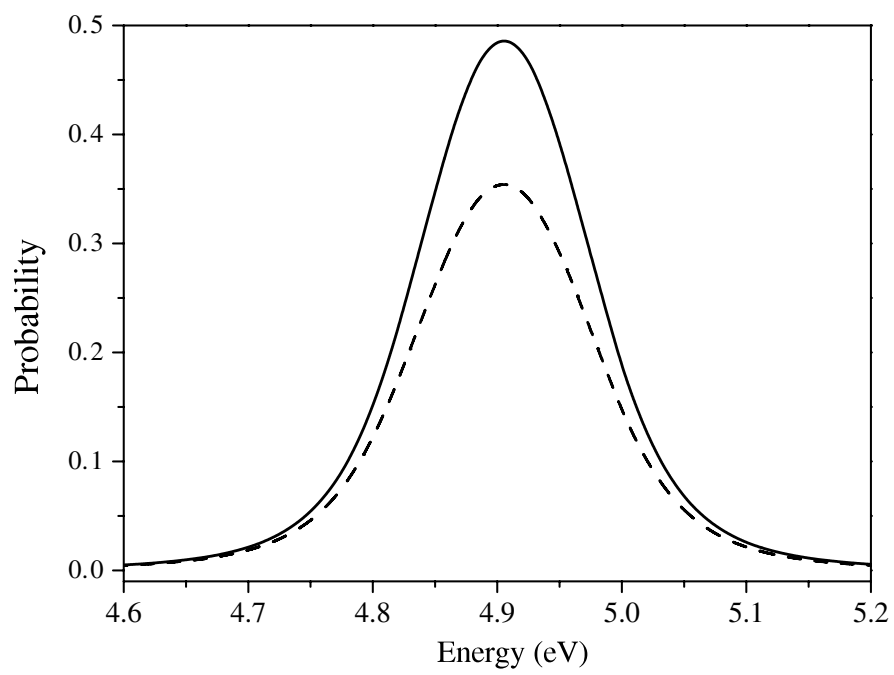

Fig. 37. Energy transfer functions obtained through the Double Difference Method (dashed line) and with the Foil Cycling Technique (continuous line).

The DDM described for a FDS could be used in a RDS as well. In this specific case, the transfer function has the form:

$$
T_{D D}(E)\left\{1-\exp \left[-N \sigma_{\gamma}(E) d_{1}\right]\right\}-\beta\left\{1-\exp \left[-N \sigma_{\gamma}(E) d_{2}\right]\right\},
$$

where the radiative capture cross section has to be used.

When applied to the RDS, the weighted difference in Eq. (29) is performed on TOF spectra acquired with the analyser close to the $\gamma$ detector's surface. Thus, differently from the FCT, in this case only $\sigma_{(n, \gamma)}(E)$ contributes to the transfer function in Eq. (29). Fig. 37 shows the non normalized transfer functions $T_{D D}(E)$ and $T_{F}(E)$ in an energy interval around the $4.906 \mathrm{eV}$ ${ }^{197} \mathrm{Au}$ resonance.

The $T_{D D}(E)$ has been calculated employing the radiative capture cross section of ${ }^{197} \mathrm{Au}$ and a value of $\beta=0.333$ $\left(d_{1}=7 \mu \mathrm{m}\right.$ and $\left.d_{2}=21 \mu \mathrm{m}\right)$.

It can be noticed that the transfer function $T_{F}(E)$, (continuous line) has a peak intensity which is a factor of about 1.4 higher than $T_{D D}(E)$ (dashed line). Thus the counting efficiency at the peak of the transfer function is about $37 \%$ higher for the FCT (for the typical values of the foil thickness employed in the two techniques). As far as the FWHM achieved with the two different techniques is concerned, it has to be mentioned that the difference is less than $5 \%$ for the case considered here.

The DDM provides a transfer function characterized by a larger depression of the off-resonance wings with respect to the FCT. In fact, while in the FCT the total and the radiative cross sections are involved (with different behaviours in the wings as shown in Fig. 34), in the DDM is the same cross section (the radiative capture one for the RD) which enters in the $T_{D D}(E)$. In the latter case the tails out of the resonance region are better subtracted as it can be seen in Fig. 38, where $T_{D D}(E)$ and $T_{F}(E)$ 


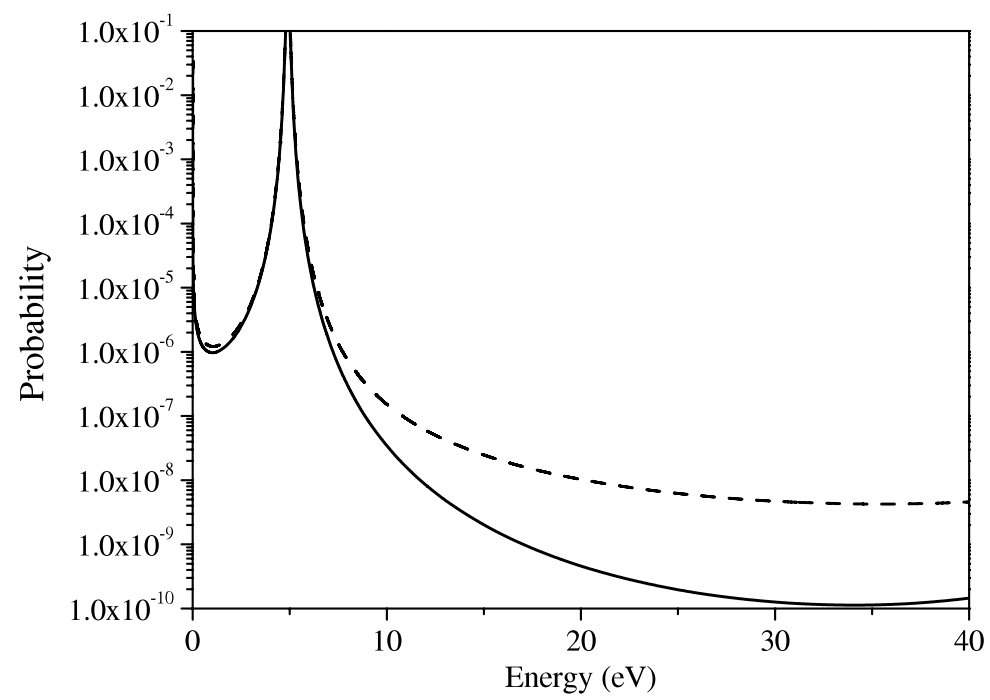

Fig. 38. Transfer functions for DDM (continuous line) and the Foil Cycling Technique (dashed line) in the energy range up to $40 \mathrm{eV}$.

are plotted, in a semi-log scale, in an extended energy region up to $40 \mathrm{eV}$. Appreciable differences can be observed above 7-8 eV, i.e. five decades below peak height: at $10 \mathrm{eV}$ the transfer function for the FCT is a factor of 4 higher than the DDM one, while at about $35 \mathrm{eV}$ it is a factor of 50 higher. However, these differences in the energy transfer functions become less pronounced when $T_{D D}(E)$ and $T_{F}(E)$ are convoluted with the instrumental resolution and the intrinsic response function of the probed system, as it will be pointed later in this report. Furthermore, the tails of the transfer functions in the DINS spectra, correspond to TOF regions characterized by very low intensity and statistically noisy data.

The combined effects of the convolution and the statistics would prevent, within the standard acquisition time on the instrument, a clear distinction (in the tail region) between data acquired using the FCT or the DDM. Thus FCT is preferred for counting rate purposes.

In fact it has to be noted that the lack of intensity in the $T_{D D}(E)$ as compared to $T_{F C T}(E)$ (see Fig. 37), results in a longer acquisition time, for the DDM, to achieve the same statistical accuracy ensured by the FCT.

\section{Gamma background sources in $\mathrm{eV}$ spectrometers}

As already stated before, Eq. (9) represents only the signal component of the RDS count rate, i.e. assuming the absence of background. A more complete expression should contain background terms, thus yielding:

$$
\widetilde{C}(t)=C(t)+B_{\text {iso }}(t)+B_{\text {dir }}(t)+B_{\widehat{z}}^{\prime}(t)+B_{\widehat{z}}^{\prime \prime}(t)+B_{f}(t)\left[\theta\left((t)-\theta\left(t-t_{0}\right)\right)\right]+B_{\text {coll }}(t)+B_{0},
$$

where $B_{\text {iso }}(t), B_{\text {dir }}(t), B_{\bar{z}}^{\prime}(t), B_{\bar{z}}^{\prime \prime}(t)$ and $B_{f}(t)\left[\theta\left((t)-\theta\left(t-t_{0}\right)\right)\right]$ and $B_{\text {coll }}(t)$ are time dependent background components correlated to the pulsed neutron beam, while $B_{0}$ describes a background term not correlated to the beam. The term $B_{\text {iso }}(t)$ represents an isotropic background mostly due to the $\gamma$ 's produced in the neutron-wall interactions in the experimental hall housing the spectrometer, caused by neutrons scattered off samples. As a matter of fact, the effect of the scatterer is to remove neutrons from the incident beam, redistributing them almost isotropically (exception is made for pure hydrogenous samples) with an intensity given by:

$$
N=N_{0} \cdot\left[1-\exp \left(-N_{s} \sigma_{s} d\right)\right],
$$

where $N$ is the number of neutrons scattered in all directions, $N_{0}$ is the number of incident neutrons (related to the incident neutron flux), $\sigma_{s}$ is the scattering cross section and $d$ is the sample thickness. The term $N_{0}$ makes the background term $B_{i s o}(t)$ correlated to the neutron beam, while the intensity is related to the sample's properties through $N_{s}, \sigma_{s}$ and $d$.

The term $B_{\text {dir }}(t)$ describes a directional component, mostly provided by the $\gamma$ 's produced in the neutron beam stop material, composed of hydrogen, iron and boron, placed at the end of the transmission beam pipe (five metres beyond the sample position in the case of the VESUVIO spectrometer at ISIS). The transmitted neutron beam is in general a high fraction of the incoming neutron beam (generally more than 70\%), so that the $\gamma$ emission from the beam stop is very intense. A large fraction of the photons are shielded from the wall material itself, but an appreciable quantity, escaping from the beam tube, may reach the detectors, especially at small scattering angles.

The terms $B_{\bar{z}}^{\prime}(t)$ and $B_{\bar{z}}^{\prime \prime}(t)$ describe background $\gamma^{\prime}$ 's generated by the moderator and the decoupler material (see Fig. 39). The subscript $\widehat{z}$ labelling the two terms in Eq. (30) evidences that these $\gamma$ are almost collinear with the beam pipe axis and travel parallel to the neutron beam. The hydrogen contained in the moderator absorbs thermal neutrons, emitting radiative capture $\gamma$ 's at $2.2 \mathrm{MeV}$ (100\% relative intensity). The moderators are surrounded by a water-cooled beryllium reflector which 


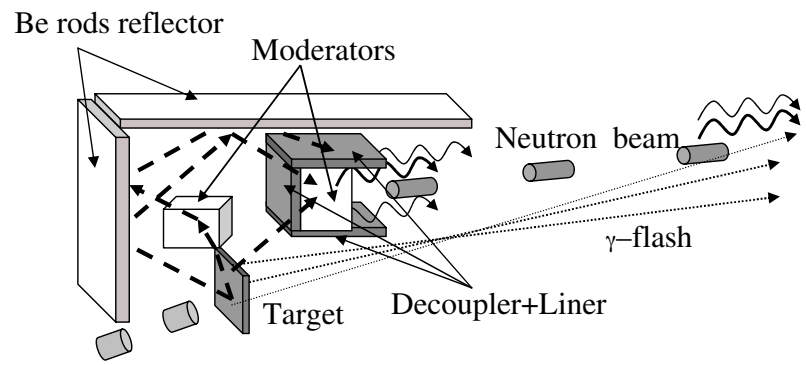

Pulsed proton beam

Fig. 39. Schematic of the target-reflector assembly: the flux of neutrons produced in the spallation reactions impinging on the moderator is enhanced by the use of the Be reflector. The pulsed neutron beams from the moderator travel together with $\gamma$-ray beams produced in the decoupler and into the moderator.

Table 6

\begin{tabular}{cl} 
Natural abundances for the naturally occurring gadolinium isotopes. \\
\hline Isotope & Natural abundance (\%) \\
\hline${ }^{152} \mathrm{Gd}$ & $0.20(1)$ \\
${ }^{154} \mathrm{Gd}$ & $2.18(3)$ \\
${ }^{155} \mathrm{Gd}$ & $14.80(12)$ \\
${ }^{156} \mathrm{Gd}$ & $20.47(9)$ \\
${ }^{157} \mathrm{Gd}$ & $15.65(2)$ \\
${ }^{158} \mathrm{Gd}$ & $24.84(7)$ \\
${ }^{160} \mathrm{Gd}$ & $21.86(19)$
\end{tabular}

scatters neutrons back into the moderators. The use of a reflector is very important to enhance the slow-neutron intensity from a moderator, although it brings about a considerable pulse broadening. In order to suppress the pulse broadening at the minimum penalty in the peak intensity, the concept of a decoupled moderator can be employed [112,2,113]. The idea is to decouple the moderator from the reflector below the so-called "decoupling energy" $E_{d}$, by inserting a slowneutron absorbing sheet, the so-called "decoupler", between moderator and reflector except for the surface viewed by the sample. With a decoupler, low-energy neutrons arriving at the moderator at later times from the reflector can be stopped from entering the moderator, thus resulting in the suppression of the pulse broadening, with the highest possible peak intensities so as to maximize the figure of merit. Cadmium $(\mathrm{Cd})$, boron carbide $\left(\mathrm{B}_{4} \mathrm{C}\right)$ and gadolinium $(\mathrm{Gd})$ are the most widely used decoupler materials. The background from the radiative capture $\gamma$ cascade from the decoupler depends on the specific material considered.

For example, on the VESUVIO spectrometer discussed before, a Gd decoupler is used. The naturally occurring isotopes of gadolinium and their isotopic abundances are listed in Table 6, while in Figs. 40 and 41 the radiative capture cross sections and the corresponding radiative capture $\gamma$ energies and relative intensities are shown, respectively.

The $\gamma$ production mechanisms in the moderator and in the decoupling system are characterized by different "relaxation times", indicated as $\tau_{M}$ and $\tau_{D}$, whose magnitudes are in the order of few tens and few hundreds of microseconds, respectively. The corresponding intensities in the time spectra decrease as $\exp \left(-t / \tau_{M}\right)$ and $\exp \left(-t / \tau_{D}\right)$. Fig. 42 shows the two components (referred to as the "moderator component" and the "decoupler component") in the time region up to $500 \mu \mathrm{s}$. This multi-components time structure in the background has been observed in DINS measurements on VESUVIO when operating in the RDS configuration $[115,116]$.

The term $B_{f}\left[\theta(t)-\theta\left(t-t_{0}\right)\right](\theta(x)$ being the Heaviside function) represents a background term relative to a transient process of $\gamma$ production occurring in the spallation target, the so called $\gamma$-flash.

The last term in Eq. (30) is present only for naturally radioactive analyser materials such as ${ }^{238} \mathrm{U}$. This background source being random in nature is not correlated to the neutron beam and thus provides a flat pedestal in the TOF spectra. Anyway the radioactive emission spectra cover a wide energy range and thus its fine characterization is useful.

The gamma background on the eV spectrometer VESUVIO was accurately investigated by performing both pulse height spectrum analysis, with a high purity Germanium (HpGe) detector, and time of flight spectra with a Yttrium-Aluminium-Perovskite (YAP) scintillator crystal [59,58]. The first was intended to recognize the most important energy regions of the gamma spectrum that contribute mostly to the overall background, the second aiming at a mapping of the background in the spectrometer's hall. Although the background measurements are of paramount importance in developing and optimizing an $\mathrm{eV}$ spectrometer, the full details of the background measurements are not essential in the context of this report. Refs. [59,58], to which the reader is referred to, will provide a thorough description of the procedure.

Here we stress that the relative weight of the different components in Eq. (30) depends on the type of measurements: for scattering measurements performed in angular range between $30^{\circ}$ and $70^{\circ}$, the effect of $B_{\text {iso }}(t)$ and $B_{\text {dir }}(t)$ is larger as compared to the others; for small-angle scattering measurements $\left(\vartheta \leq 10^{\circ}\right)$, the relative weight of $B_{\bar{z}}^{\prime}(t), B_{\bar{z}}^{\prime \prime}(t)$ and $B_{d i r}(t)$ become comparable to that of $B_{i s o}(t)$ that maintain almost the same magnitude in both scattering configuration. 

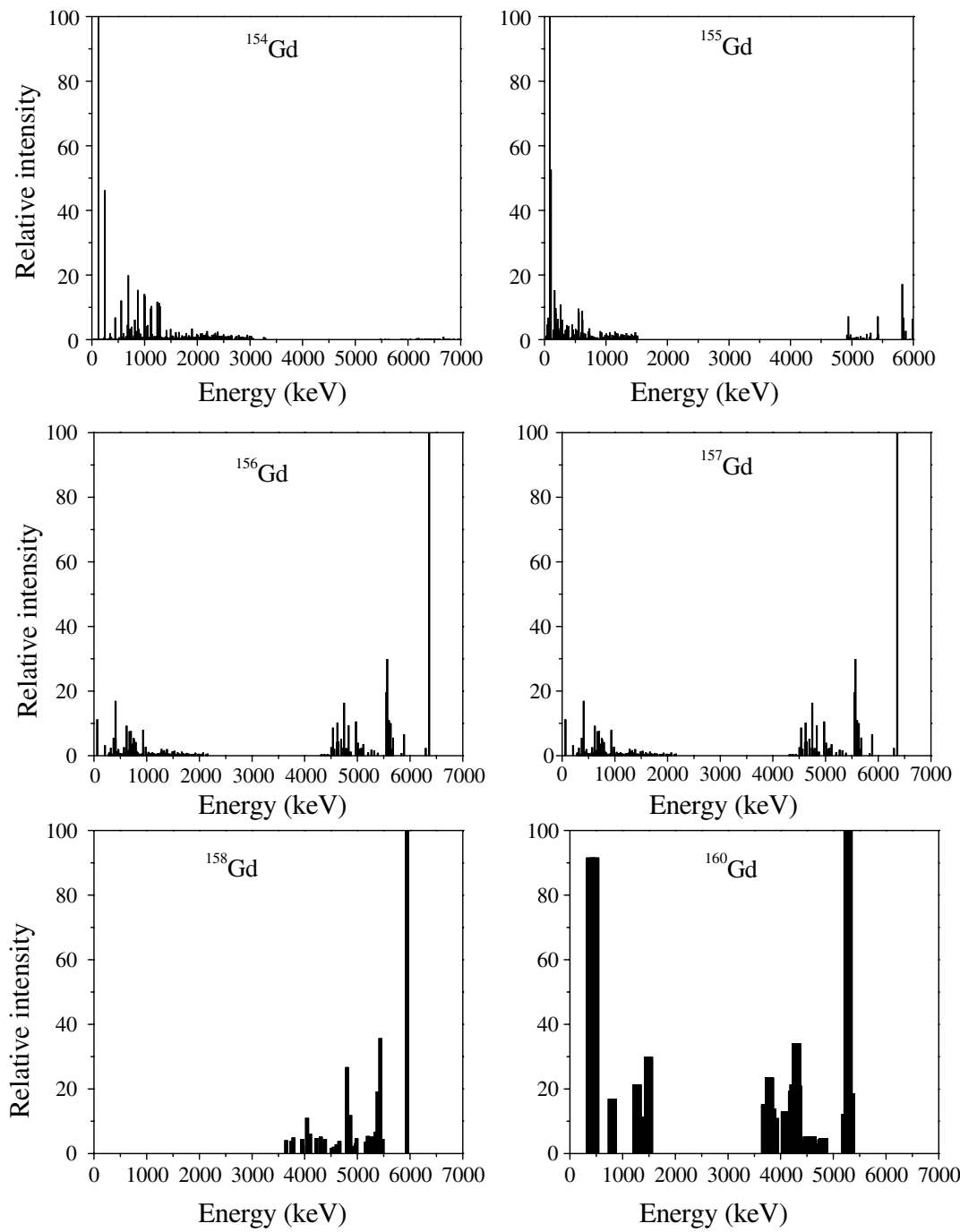

Fig. 40. Energies and relative intensities of the radiative capture $\gamma$ rays from the Gd isotopes [114].

The term $B_{\text {coll }}(t)$ is appreciable especially for backscattering experiments, as in this case the detectors are closer to the incident beam collimation system.

\section{Applications}

In this section we will briefly overview some applications of eV neutron spectroscopy, providing the main details of the theoretical framework, outlining some important results and/or potential achievements, highlighting in a few cases the R\&D activity for novel instrumentation.

\subsection{Deep inelastic neutron scattering}

Deep inelastic neutron scattering (DINS) is a unique experimental technique providing useful information on the shorttime dynamics of nuclei in a series of condensed matter systems [41,11,13,40,22,12,117]. A remarkable development of the technique occurred since middle eighties, when intense fluxes of epithermal neutrons were made available from spallation pulsed neutron sources. The inverse geometry spectrometers, described in detail in this report, can reach unlimited energy (loss), $\hbar \omega$, and wave vector, $q$, transfers, as shown in the previous sections. Although a comprehensive and thorough discussion of DINS is made in Ref. [41], it is worth to remind here some key relations that are useful for the brief overview presented in this section.

In the DINS regime, the inelastic neutron scattering cross section for unpolarized neutrons is related to the dynamic structure factor $\mathrm{S}(\mathbf{q}, \omega)$ by $[118,119]$ : 

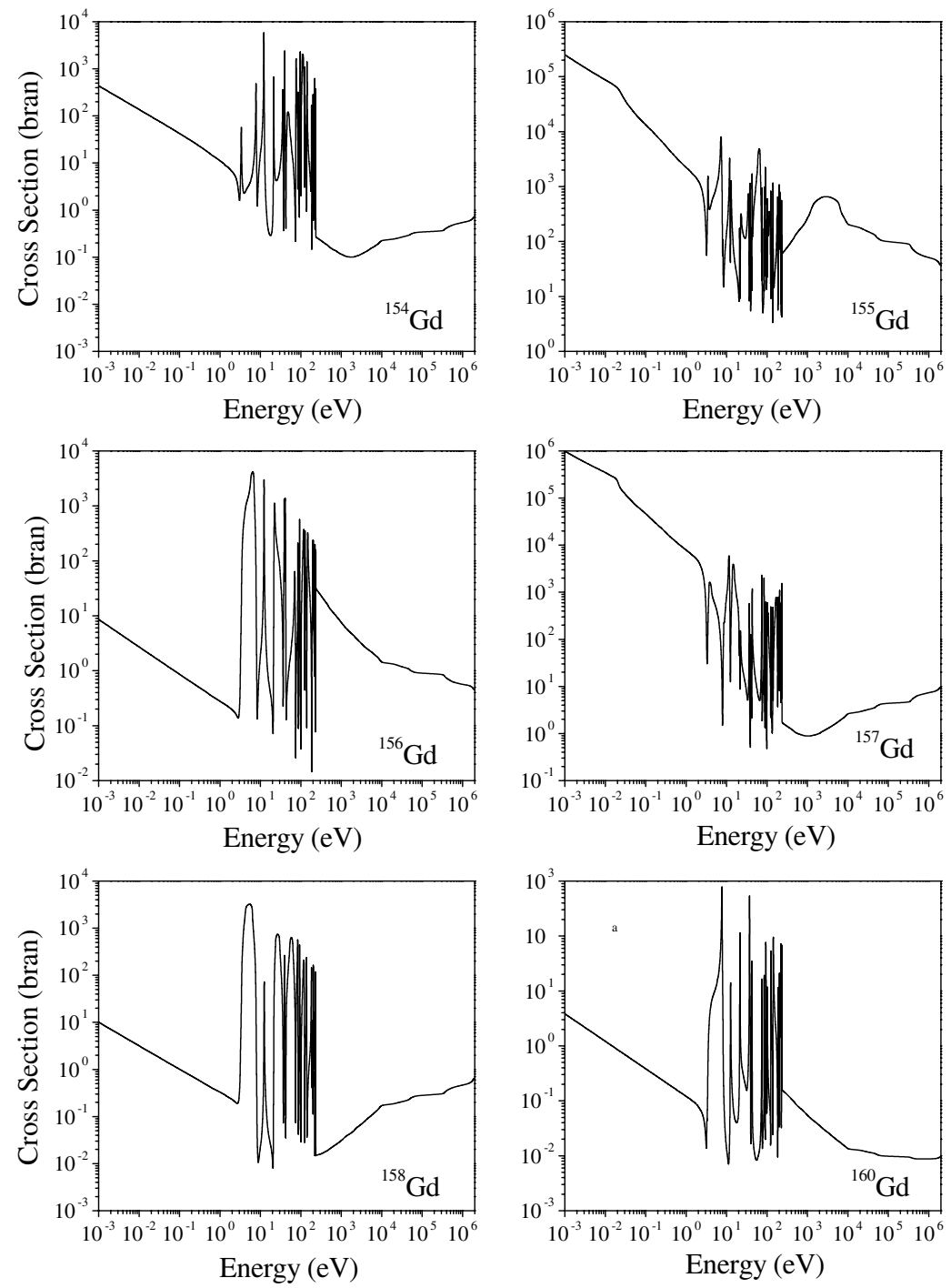

Fig. 41. Radiative capture cross-sections of different Gd isotopes, plotted in the $1 \mathrm{meV}-1 \mathrm{MeV}$ energy region [114].

$$
\frac{d^{2} \sigma\left(E_{0}, E_{1}, \vartheta\right)}{d \Omega d E_{1}}=\hbar^{-1} \sqrt{\frac{E_{1}}{E_{0}}}\left[|b|^{2} S(\mathbf{q}, \omega)+\left(\left|b^{2}\right|-|b|^{2}\right) S_{I}(\mathbf{q}, \omega)\right],
$$

$b$ and $S_{I}(q, \hbar \omega)$ being the scattering length of the probed nucleus and the incoherent contribution to the total dynamic structure factor, respectively. $E_{0}, E_{1}$ and $\vartheta$ are the incident neutron energy, the scattered neutron energy and the scattering angle, respectively. At high $q$ values (typically above $20 \AA^{-1}$ ), the scattering is incoherent, meaning that it occurs from a single particle. The typical values of the energy transfer $\hbar \omega$ attainable in DINS experiments range from 1 to $100 \mathrm{eV}$, corresponding to a time scale of the order of $10^{-15}-10^{-17} \mathrm{~s}$, which is much shorter than the characteristic time scales of the high energy excitations in condensed matter (typically above $\tau \simeq 10^{-15} \mathrm{~s}$ ). Under these kinematical conditions, the nucleus probed by the neutron recoils freely $[41,40]$. Thus, DINS explores the so-called short-time selfdynamics, and the incoherent and free recoil scattering (resembling that occurring in the Compton scattering of hard X-rays off electrons) manifests in the well-known impulse approximation (IA). Within the IA, the inelastic neutron scattering cross-section in Eq. (32) is [41,120]:

$$
\frac{d^{2} \sigma\left(E_{0}, E_{1}, \vartheta\right)}{d \Omega d E_{1}}=\hbar^{-1} \sqrt{\frac{E_{1}}{E_{0}}}\left|b^{2}\right| S_{I A}(\mathbf{q}, \omega),
$$

while the dynamic structure factor is given by [119]:

$$
S_{I A}(\mathbf{q}, \omega)=\hbar \int n(\mathbf{p}) \delta\left[\hbar \omega-\hbar \omega_{r}-\frac{\mathbf{p} \cdot \hbar \mathbf{q}}{M}\right] d \mathbf{p},
$$




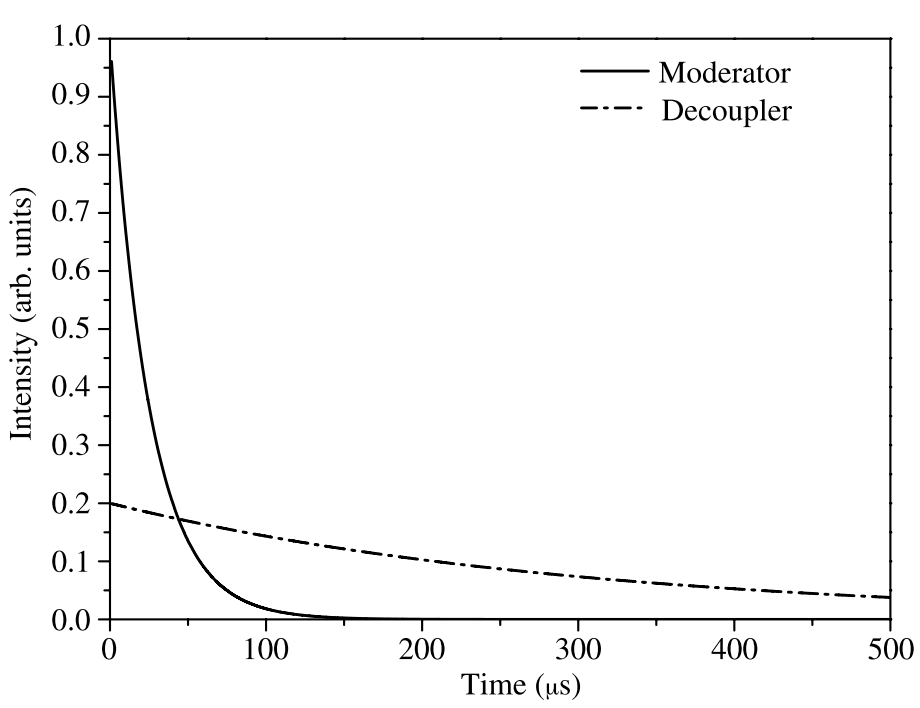

Fig. 42. Plot of $B_{\widehat{z}}^{\prime}(t)$ and $B_{\widehat{z}}^{\prime \prime}(t)$ in Eq. (30). The amplitude of the moderator component is normalized to 1, while the amplitude of the decoupler component is chosen as a fraction of the first one.

$n$ (p) being the single particle momentum distribution. Eq. (34) establishes that scattering occurs between the neutron and a single particle, while also conserving kinetic energy and momentum of the particle + neutron system. The term $\hbar \omega_{r}=\frac{\hbar^{2} q^{2}}{2 M}$ is the recoil energy, i.e. the kinetic energy the struck particle would have, providing it was stationary before the collision and absorbed all the momentum transferred by the neutron [23]. The asymptotic property of the dynamic structure factor, which was first emphasized by West in the context of electron scattering [118], provides the basic frame for its application to DINS. Early experimental studies at spallation neutron sources showed that for wave vector transfers above $30 \AA^{-1}$ the scattering was centred at the recoil energy given by the impulse approximation, thus showing that the energy transfer and the recoil energy are not independent in this kinematical range [121]. Within the framework of the IA, $\hbar \omega$ and $q$ are then explicitly coupled through the West scaling variable $y$, defined as [118]:

$$
y=\frac{M}{\hbar^{2} q}\left(\hbar \omega-\hbar \omega_{r}\right) .
$$

Eq. (34) can then be reduced to the form

$$
S_{I A}(\mathbf{q}, \omega)=\frac{M}{\hbar q} J(y, \hat{q}),
$$

where

$$
J(y, \hat{q})=\hbar \int n\left(\mathbf{p}^{\prime}\right) \delta\left(\hbar y-\mathbf{p}^{\prime} \cdot \hat{q}\right) d \mathbf{p}^{\prime}
$$

is the Neutron Compton Profile (NCP), formally defined as the Radon transform of the momentum distribution. The quantity $\hat{q}$ is a unit vector, as $J(y, \hat{q})$ no longer depends on the magnitude of q. The function $J(y, \hat{q}) d y$ is the probability for an atom to have a momentum parallel to $\hat{q}$ of magnitude between $\hbar y$ and $\hbar(y+d y)$.

For an isotropic system, the direction $\hat{q}$ is immaterial and Eq. (37) becomes [41]:

$$
J(y)=2 \pi \hbar \int_{|\hbar y|}^{\infty} p n(p) d p .
$$

It has to be stressed that in the IA framework, $J(y)$ is symmetric and centred at $y=0$ and the relation between $n(p)$ and $J(y)$ is [41]:

$$
n(p)=-\frac{1}{2 \pi \hbar^{3} y} \cdot\left[\frac{d J(y)}{d y}\right]_{h y=p} .
$$

It is worthwhile mentioning that the IA is strictly valid only in the asymptotic double limit $(q, \hbar \omega) \rightarrow \infty$, keeping $y$ constant. For finite values of the energy and wave vector transfers, the dynamical structure factor retains an additional dependence on $q$ which is known as final state effects (FSE). A detailed description of this contribution can be found in Refs. $[122-125,41,40]$.

In the context of DINS measurements on condensed matter systems, it is possible to discern two separate but interwoven threads of development within the last decade. In the first place, systematic studies of the zero-point atomic kinetic energies 


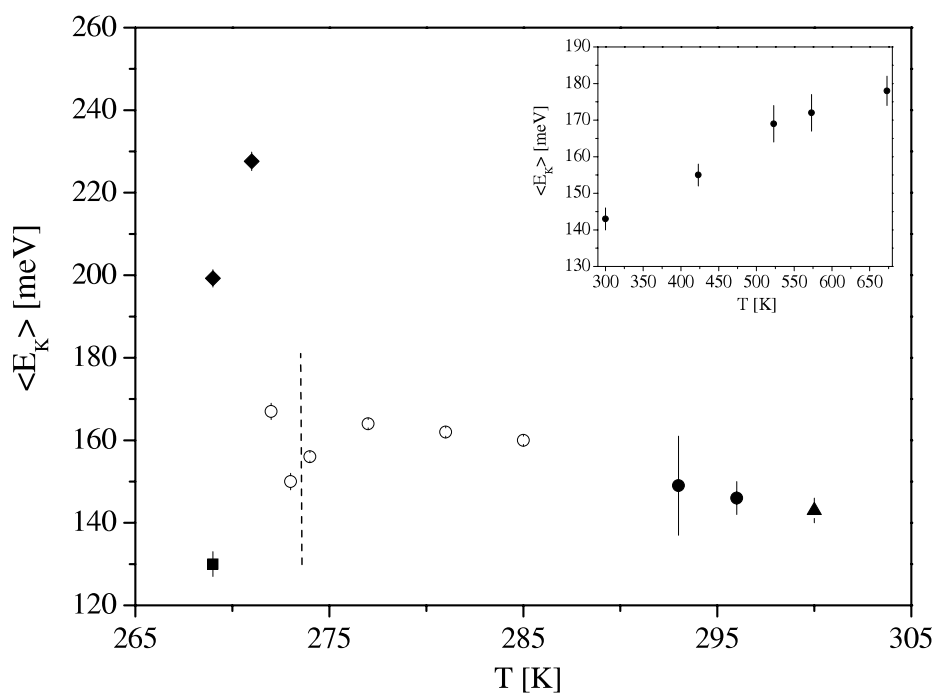

Fig. 43. Proton mean kinetic energy, $\left\langle E_{K}\right\rangle$ in water as a function of temperature. Source: From Ref. [126].

allow an insight into the relevance of quantum effects in the energetics of monoatomic and molecular systems. In the second place, accurate studies of momentum distribution line shapes provide unique information on the local environment and potential energy surfaces. DINS measurements performed on the VESUVIO spectrometer, both operated as a RFS or a RDS provided relevant results in the field of molecular H-bonded systems as well as monoatomic quantum systems (e.g. ${ }^{3} \mathrm{He},{ }^{4} \mathrm{He}$, etc.). Indeed the behaviour of protons, and more generally of light nuclei in condensed phases, is significantly affected by quantum effects even at ambient temperatures. The isotopic effect in water, the ferroelectric behaviour of potassium diphosphate, and the formation of high pressure ice phases, are just a few of the relevant phenomena where the quantum behaviour of the nuclei plays a role. DINS measurements allow to give evidence of quantum effects by strong deviations of the measured momentum distribution from the classical Maxwell distribution. This technique provides complementary information to what is garnered from diffraction techniques that measure the spatial correlations among the nuclear positions. Because of the non-commuting character of position and momentum operators in quantum mechanics, the momentum distribution is sensitive to the local environment. In the case of water, the most important hydrogenbonded system, chemical interactions occurring in the bulk typically represent small changes in the energy of constituents, compared to the energy sequestered in the zero point motion of the protons in the water, primarily in that of the stretch mode. To the extent that this energy does not change in the interaction, it may be ignored, and usually is. Nearly all simulations of water in a variety of settings are done with models of water for which these changes cannot occur, or if they can, are not considered because of the additional expense of treating the protons quantum mechanically. The energy does change, however, as the structure of the hydrogen bond network changes. DINS allows to observe the changes in zero point kinetic energy directly. These changes can be large $\left(\geq k_{B} T\right)$, and in either direction. For example, systematic studies of the proton mean kinetic energy in bulk liquid water from the supercooled metastable phase to supercritical conditions highlight the connection between the proton short-scale response to the changes in the H-bond network [126-132,115, 133-136].

More interestingly, the details of the shape of the momentum distribution correspond to details of the confining potential for the target particle (proton, deuteron. etc.): indeed the momentum distribution of the protons is a direct reflection of the structure of their local environment, and in favourable circumstances, it can be used to infer the Born-Oppenheimer (BO) potential for the motion of the proton. When the temperature is low enough, so that the target particle is in its ground state, and if it is a light ion such as hydrogen, and surrounded by heavy ions, many body effects involving the motion of the heavy atoms can be neglected. The environment of the proton is then described by means of a one particle potential. If the site of the scatterer is inversion symmetric, it is then possible to reconstruct the spatial wave function and the BO potential of the scattering ion. This approach allowed, for example, to obtain a direct observation of coherent proton tunnelling in potassium diphosphate (KDP) and the first direct measurement of the proton 3-D BO potential in any material [137,25,24,138-140]. In all cases, it puts strong constraints on any model of the potential surface (see Figs. 43-46).

Similarly to the case of molecular systems, in monoatomic and diatomic quantum liquids and solids, the investigation of zero-point kinetic energies and momentum distribution line shapes provide unique information on momentum quantum fluctuations due to effective single particle confinement in an interacting quantum liquid [141,142], as well as long range coherence such as the condensation at zero momentum state in superfluid helium, or the signatures of the Fermi-Dirac discontinuity in liquid ${ }^{3} \mathrm{He}$. In the case of DINS studies on helium, the measurements with $\mathrm{eV}$ inverse instruments complement those carried out using direct geometry, such as the MARI spectrometer at ISIS, where, at the expense of a wave 


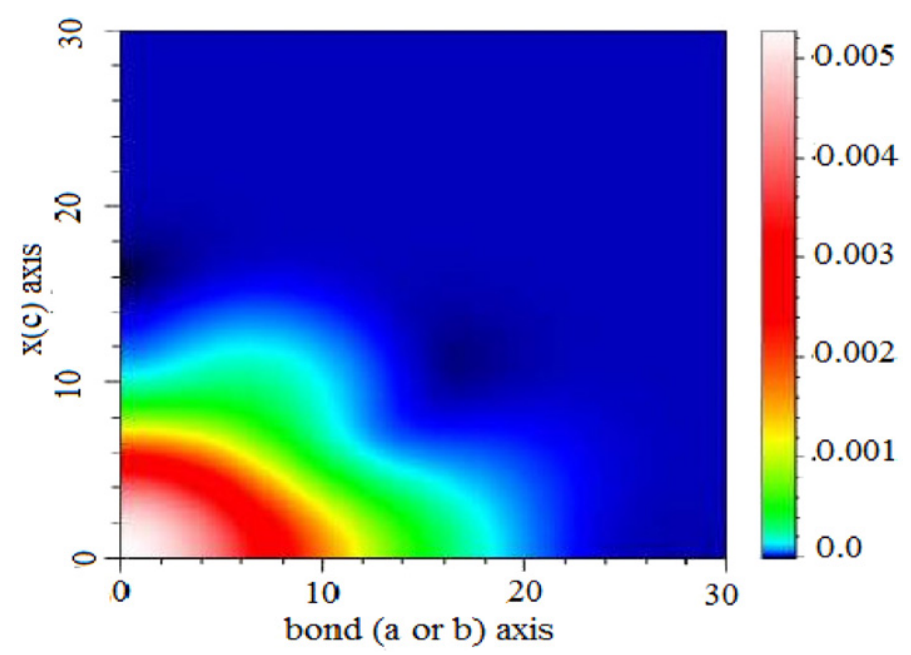

(a) KDP momentum distribution $T=90 \mathrm{~K}$.

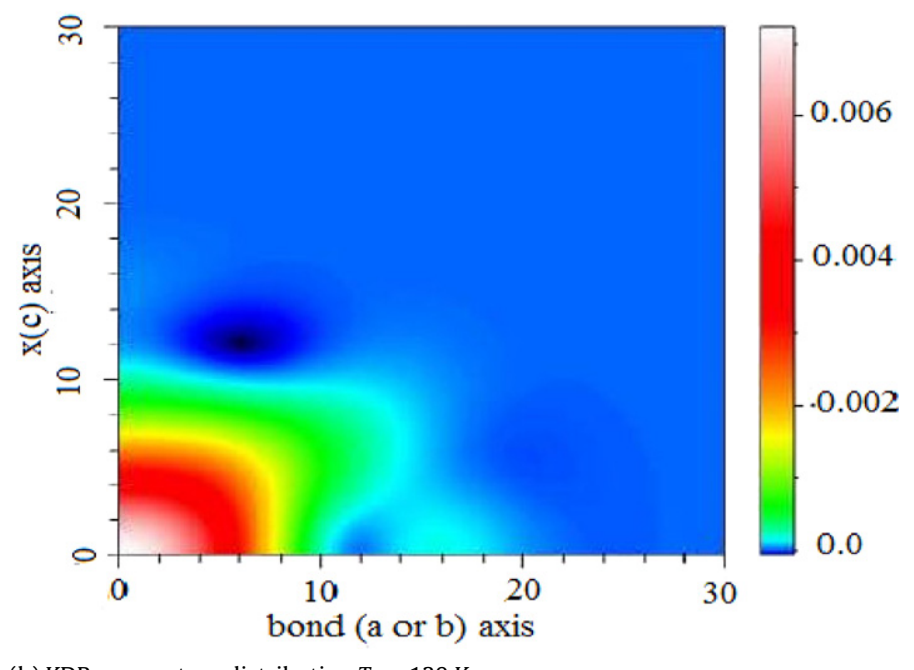

(b) KDP momentum distribution $T=130 \mathrm{~K}$.

Fig. 44. Two-dimensional proton momentum distribution above and below the ferroelectric transition. Source: From Ref. [137].

vector transfer below $30 \AA^{-1}$, the resolution for scattering from helium is excellent [143-145,70,146]. It has to be stressed that, in contrast to the potential energy, the kinetic energy of a many-body system has distinctly different properties in the classical regime (Maxwellian), the quantum liquid, the crystal (Debye-like), the superfluid (Bose condensation), and for liquid ${ }^{3} \mathrm{He}$ (Fermi-liquid behaviour). Helium and neon are nearly ideal systems in this respect, being amenable for a tuning of nuclear quantum effects, thanks to the wide range of densities, temperatures, and phases that can be achieved experimentally and retained stably in the laboratory. These systems, together with the other molecular quantum fluids such as $\mathrm{H}_{2}$ and $\mathrm{D}_{2}$ have been extensively investigated in terms of the zero-point mean kinetic energy dependences on temperature, density, and nano-size confinement [147-160].

For systems described by quantum statistics, the shape of the momentum distribution provides an insight into the relevance of the spin-statistics behaviour. An outstanding example is the low-temperature superfluid behaviour of liquid ${ }^{4} \mathrm{He}$, where the superfluidity is associated with Bose condensation of a macroscopic fraction of the helium atoms into a zero-momentum state $[12,23,142,141,161]$. A peculiar characteristics of the experimental DINS determination of $n(p)$ is that a quantitative comparison with theories is possible. In fact, in those systems where the interatomic or intermolecular interaction potential is known to a high degree of accuracy, such comparison has been extensively carried out for monoatomic quantum liquids and solids, such as ${ }^{4} \mathrm{He}$ and ${ }^{3} \mathrm{He}$ and neon $[147,153,162,150,163]$.

Finally, we would like to remark that, in the last five years, synergistic advances have been accomplished in both experimental and theoretical approaches for the determination of momentum distribution in hydrogen-bonded systems, 

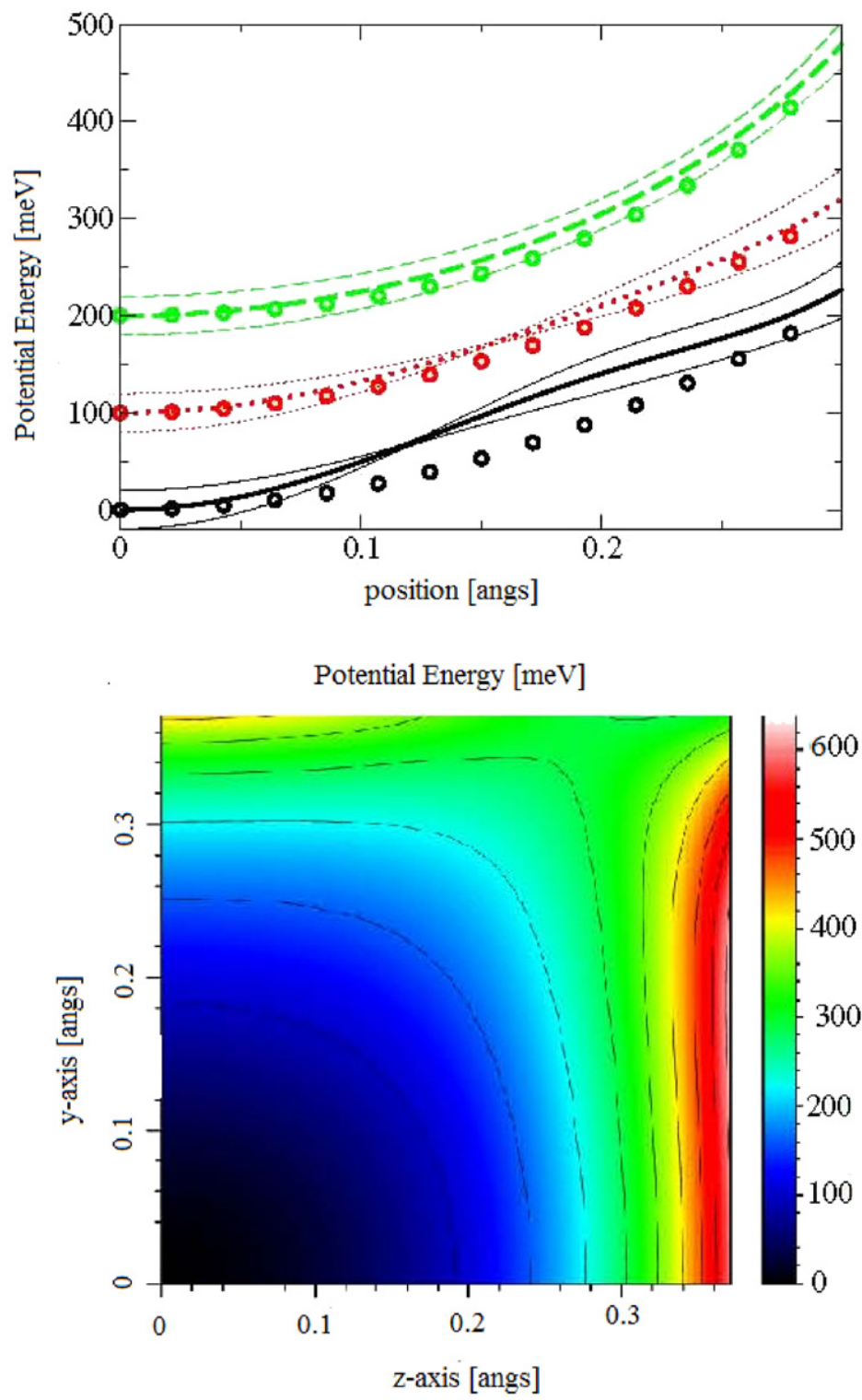

Fig. 45. DINS-derived Born-Oppenheimer potential for protons in $\mathrm{Rb}_{3} \mathrm{H}\left(\mathrm{SO}_{4}\right)_{2}$ at $10 \mathrm{~K}$ [24]: (a) along the $x$-axis (solid line), $y$-axis (dotted line), and $z$-axis (dashed line), the curves are shifted by $100 \mathrm{meV}$ along the vertical axis for clarity. The uncertainties are shown in dashed lines. The fit to a double Morse potential are shown in circles. (b) The potential energy surface in the $y z$ plane.

and water in particular. These have been carried out thanks to the R\&D activity on detection and energy analysis methods (see the Resonance Detector and the Foil Cycling techniques described before), that resulted in considerable improvements on the resolution and signal to background ratio for proton $n(p)$ studies. On the theoretical side, the development of novel quantum Monte Carlo simulation approaches has allowed an unprecedented description of nuclear quantum effects on the momentum distribution in water [164,26,165-168].

\subsection{High-energy inelastic neutron scattering}

By virtue of its magnetic moment, the neutron can scatter off unpaired electrons through electromagnetic interaction with the electron spin. There are several types of experiments in the field of condensed matter involving energy transfers in the $\mathrm{eV}$ region $(\hbar \omega>0.5 \mathrm{eV})$, beyond the deep inelastic experiments discussed above $[169,146]$. Such experiments include the study of high (anharmonic) vibrational states in hydrides [170], of electronic transitions in rare earth metals and compounds [90], in molecules and insulators [171], semiconductors [172-175] and magnetic materials [176,177,82,178-180, 89,91]. Moreover, studies of the dynamics of paired electrons of inner filled $s^{2}$ shells of impurity atoms in metals, where the scattering is due to single electron excitations from the inner shell of the impurity to the free states of the metal, have been 


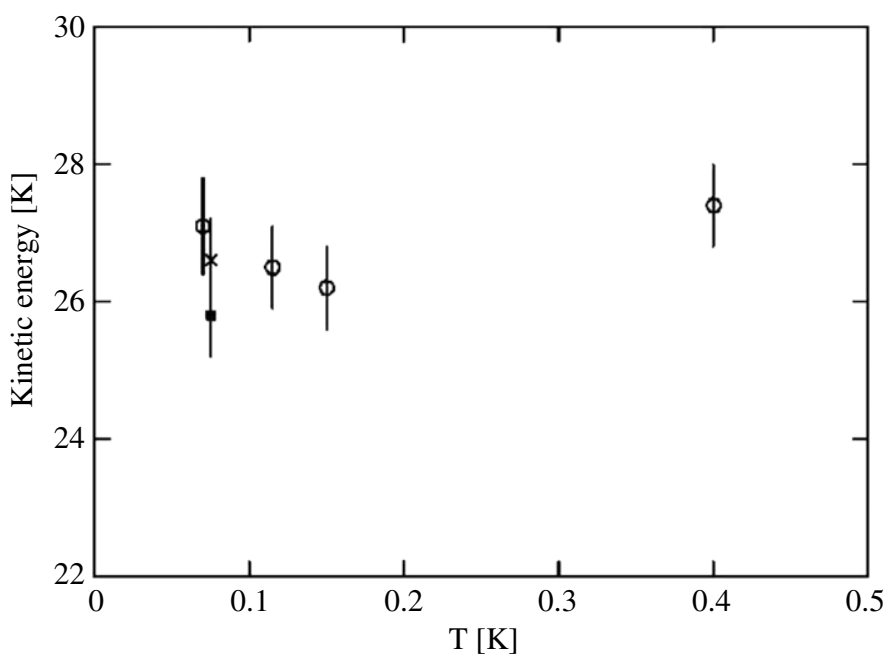

Fig. 46. Atomic kinetic energy in solid ${ }^{4} \mathrm{He}$ as a function of temperature across the supersolid transition [159].

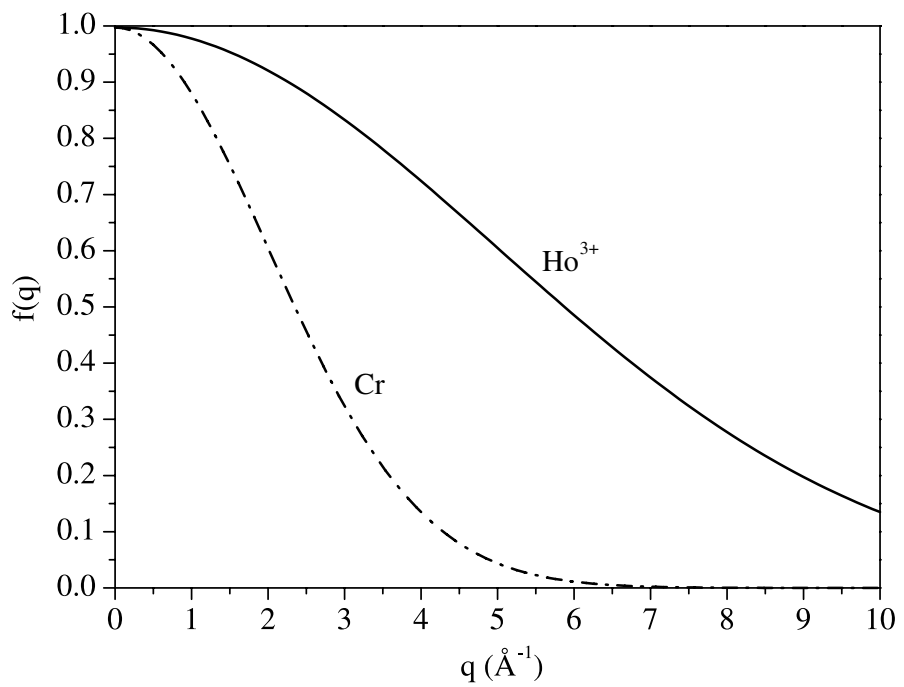

Fig. 47. Magnetic form factors of $\mathrm{HO}^{3+}$ and $\mathrm{Cr}[28,91]$.

recently considered on the theoretical ground [181]. In order to fulfil the kinematic requirements imposed by the decrease of the magnetic scattering intensity with the square of the magnetic form factor $f(q)$ see Fig. 47 , as $q$ increases, high incident and final energy, coupled to low scattering angles have to be employed to access the high $\hbar \omega$ and low- $q$ range [182].

Epithermal neutron beams at $\mathrm{eV}$ beam lines are characterized by a non-uniform spatial intensity profile. For example, the Vesuvio spectrometer is characterized by an umbra of $3 \mathrm{~cm}$ in diameter, penumbra of $5 \mathrm{~cm}$ diameter, at sample position, and a divergence of approximately $0.3^{\circ}$. These impose the instrumental layout [30]. The latter is characterized by a very low angle detector bank (VLAD) with scattering angles in the range $1^{\circ} \leq \vartheta \leq 4^{\circ}$, and secondary flight paths of approximately $200 \mathrm{~cm}$ [183]. The single detector count rate is therefore lower than in the DINS case due to: (1) reduced scattering cross sections for magnetic scattering; (2) reduced solid angle subtended by the detector array. The low angle bank on Vesuvio provides the possibility of observing high energy excitations $(0.5-10 \mathrm{eV})$ at modest wave vector transfer $\left(2-10 \AA^{-1}\right)$ in materials. At present, the effectiveness of the VLAD layout is reduced due to the non-optimized signal to background ratio [59]. However the main components of the background field have been well identified [58,59]. This implies that the necessary modifications to optimize the signal to background ratio can be implemented by, for example, the use of the Foil Cycling technique [110,111], directional shielding against the line of view of the moderator and/or beam dump, neutron time of flight-photon energy bi-parametric data recording $[184,185]$. These actions can be easily addressed in any forthcoming upgrade on VESUVIO, as well as giving the ground for the low angle bank on eV spectrometers that have been proposed at last-generation spallation sources such as the Spallation Neutron Source (USA). 


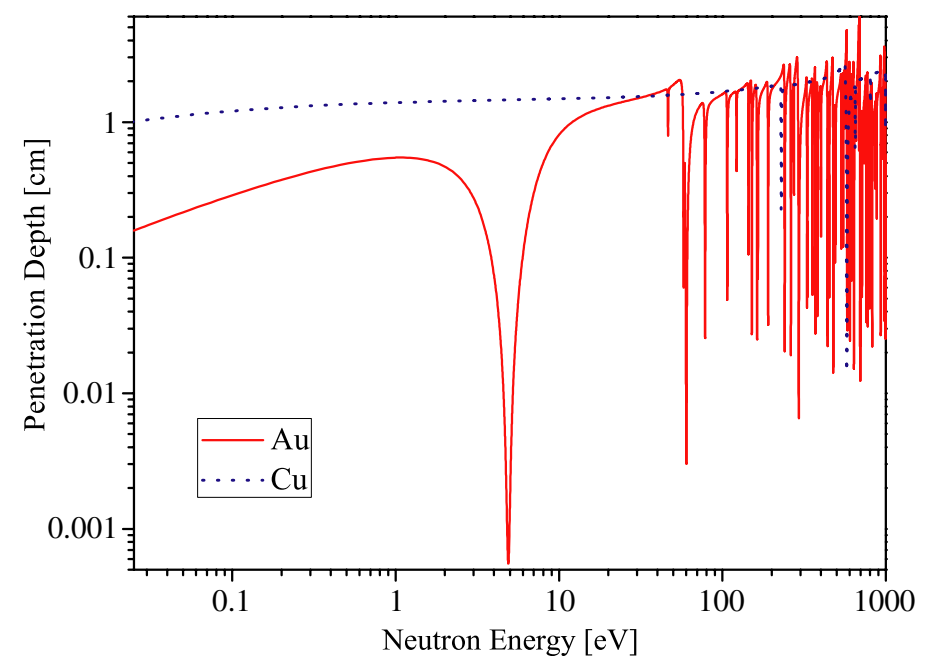

Fig. 48. Neutron energy-dependent penetration depths in natural gold and copper [109].

\subsection{Application to cultural heritage studies}

Beyond DINS and HINS, epithermal neutron beams in the range 1-1000 eV can be also exploited for the study of the elemental composition of materials (especially metals) of cultural and artistic relevance. Indeed, many elements in the periodic table possess neutron cross sections characterized by the presence of resonances (absorption and radiative capture) in the above range. Moreover, different isotopes of the same element have different resonance energies, all of them being fingerprints of an element within the sampled object. These properties offer unique advantages to the study of metals in bulk and macroscopically inhomogeneous objects, as well at the level of impurities and/or traces. Making use of the energy dependent neutron cross sections for a variety of metals of interest in cultural heritage objects, such as gold, copper, silver, etc., it is possible to probe complex objects non-destructively. This can be done exploiting the neutron penetration depths, that in general are orders of magnitude larger than the typical photon/particle probes [186-188]. Fig. 48 reports the neutron energy-dependent penetration depths in natural gold and copper, showing values ranging between a few microns (resonance regions) to several centimetres in the energy range $25 \mathrm{meV}$ to $1 \mathrm{keV}$.

At a neutron time of flight instrument, the resonances appear in the TOF spectrum at time positions that are univocally defined by the resonance energy and the primary neutron flight path $L_{0}$.

Two possible configurations may be exploitable: (1) register the prompt $\gamma$ rays produced upon resonance absorption by means of a photon detector to assign the arrival time of the resonant neutron onto the sample. This technique is known as Neutron Resonance Capture Analysis (NRCA) [189,33,32]. The TOF spectrum in NRCA is featured by peaks: the peak time position provides a qualitative information (i.e. it identifies the element), its area provides information on the amount of the particular element identified within the object. This technique, already exploited to investigate for example the mechanism of $s$-wave and $p$-wave neutron resonance capture in light and medium-weight nuclei [107], is fruitfully used at the GELINA [50] pulsed neutron source (Belgium). Recently NRCA was also performed with excellent results at the ISIS source on the INES beamline, as shown in Fig. 49 [190-192].

In the second configuration, the resonance absorption is used by analysing the beam transmitted through the sample and using a neutron counter based on the reactions listed in Section 1. When a white neutron beam passes through a bulky sample, and in correspondence of the resonance energies the beam is strongly attenuated. Within the EU-FP VI funded ANCIENT CHARM project $[193,194]$ this approach was extensively tested to develop an imaging technique, namely the Neutron Resonance Transmission Imaging (NRTI). For example, using NRTI, bi-dimensional elemental maps of composite copper-gold metallic objects were obtained with a $2 \mathrm{~mm}$ spatial resolution (see Figs. 50 and 51).

NRCA can be upgraded as an imaging tool to map the elemental/isotopical composition of an object [34]. This can be achieved by using a pencil neutron beam $\left(\mathrm{mm}^{2}\right.$ area) and a multi-positioning of the irradiated object with respect to the beam with a $x y z-\omega$ motorized table [196]. The techniques and methods presented here have the potential to meet the requirements for next generation energy-selective neutron imaging with sub-millimetre spatial resolution [197].

\subsection{Exploiting the edge of the spallation neutron spectrum: the Chip Irradiation case}

Although not specifically related to $\mathrm{eV}$ neutrons instrumentation, it is worth to stress that thanks to the intense fluxes of both epithermal and fast neutrons $(E \geq 1 \mathrm{MeV})$, spallation neutron facilities offer a unique opportunity for the investigation of the so-called Single Event Effects (SEE) in electronics. These occur when the high energy component of the terrestrial neutron field interacts with the sensitive region of a Si-based chip, disrupting its correct operation. This is a concern in fields 


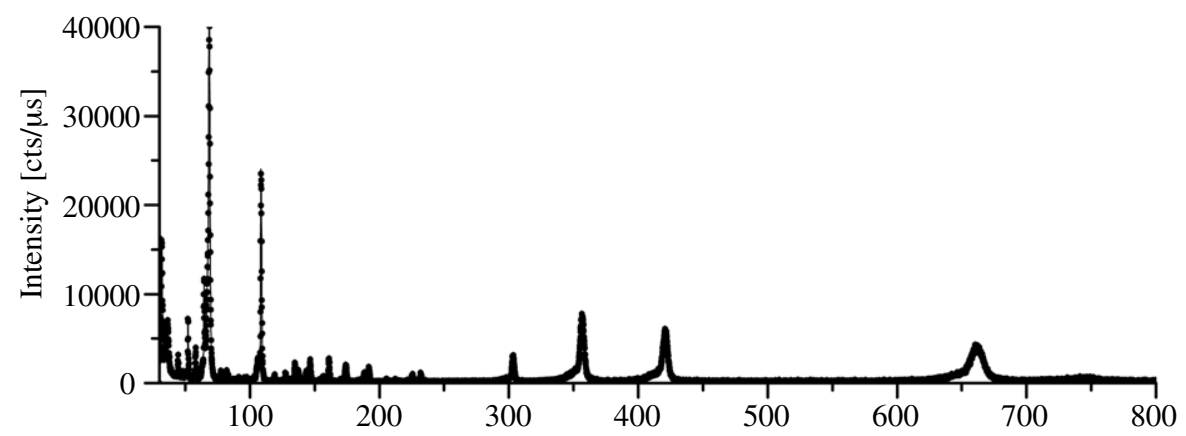

Time of flight $[\mu s]$

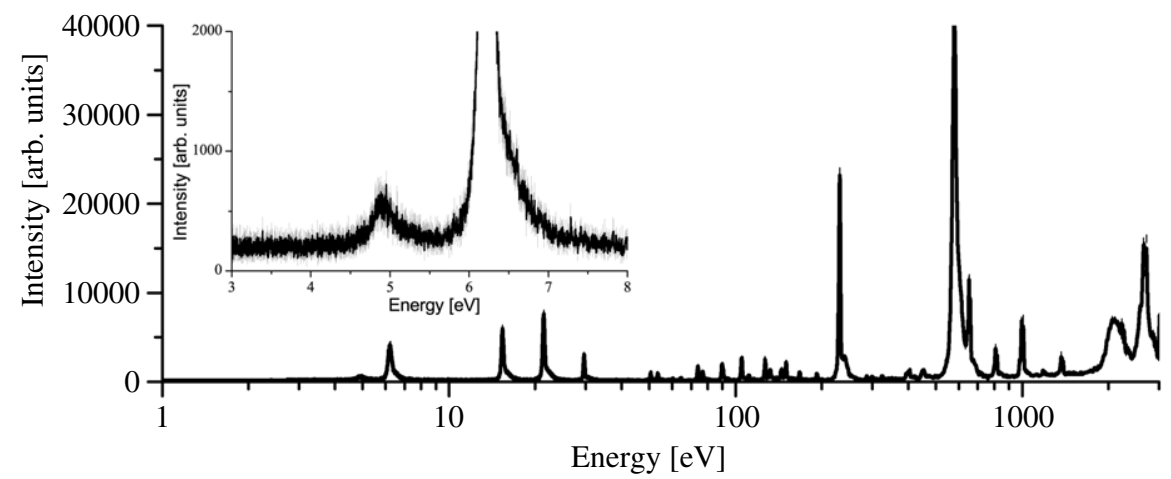

Fig. 49. NRCA spectrum (neutron time of flight in upper panel, incident neutron energy in lower panel) of a $\mathrm{Cu}_{92} \mathrm{Sb}_{8}$ sample with $40 \mathrm{ppm}$ (by weight) of Au impurities, measured on the INES beam line at ISIS. The inset shows the energy region around the $4.908 \mathrm{eV}$ gold resonance [189].

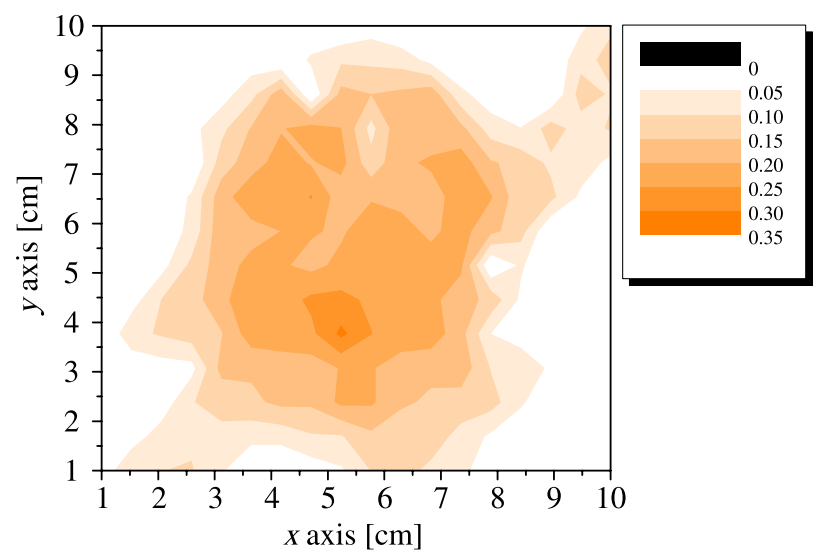

Fig. 50. Two-dimensional elemental map for copper in a composite copper-gold reference sample [195].

such as automotive, aviation, information technology infrastructures and medical [198-201] related to the development of novel chips featuring nanometric size devices. SEE tests in the atmospheric neutron field (see Fig. 52) take several months of data recording time.

Spallation neutron sources produce an almost atmospheric-like neutron spectrum with intensities that are, on the average, $10^{4}-10^{5}$ higher than the atmospheric one, allowing to perform tests in a few minutes. Typically, SEE tests were mostly performed at LANSCE (US) or TRIUMF (Canada), but since 2005, after successful tests performed at the VESUVIO spectrometer [204], the effectiveness of the ISIS source for SEE investigation was shown [205]. This opened the way to the design of the ChipIr beam line at ISIS TS2 [206].

At present an R\&D activity is devoted to the development of fast neutron real time detection techniques. Different approaches are under study, such as the Bonner Sphere Spectrometer [1], Thin Film Breakdown Counters (TFBC) [207], or diamond detectors [208-212], the last one allowing for very localized ( $\mathrm{mm}^{2}$ resolution) beam monitoring. Together with detector-oriented activities, a study of the spallation target is ongoing in order to fulfil the requirement of high intensity 


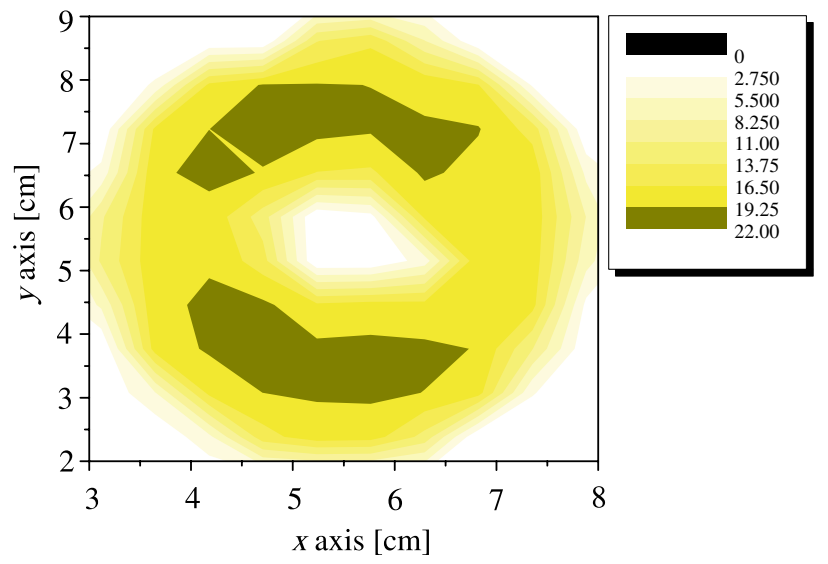

Fig. 51. Two-dimensional elemental map for gold in a composite copper-gold reference sample [195].

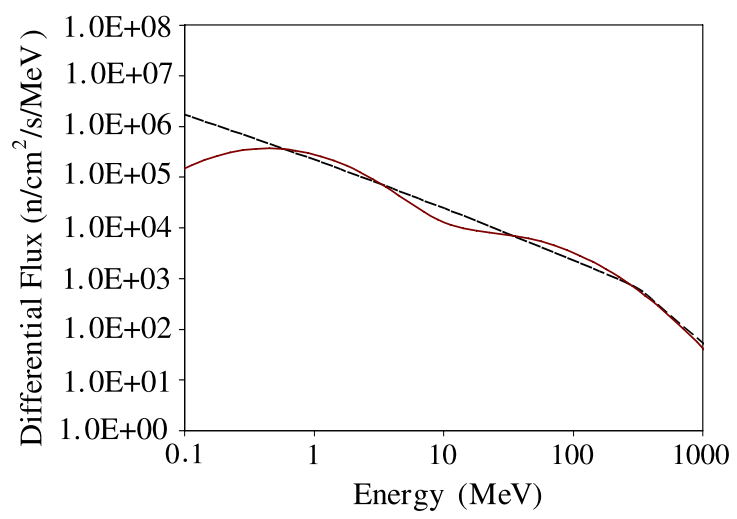

Fig. 52. Atmospheric neutron spectrum according to the IEC standard (dashed line) [202] and JEDEC standard (continuous line) [203].

of fast neutrons. This may offer a benefit also for electron volt neutron beams for the applications mentioned above in this section.

\section{Conclusions}

In many fields of experimental physics the breakthroughs in the fine knowledge of matter have been brought into reality through advances in the development of new detector concepts and devices for energy analysis of radiation beams. In the case of neutron scattering, the pioneering work carried out by Samosvat et al. [22], was not immediately followed by other experimental studies, until the construction of pulsed neutron sources was completed. Indeed more complex experiments, but still on an explorative level, were carried out on model systems during the first years of operation of pulsed sources $[16,17,20,15,213]$. These were not corroborated by a comprehensive theoretical background, indeed in its early stages at the time, neither at the instrumental level, having the character of a limited research and development activity that did not raise from test level towards a full scientific programme. The instrumental apparatus where the scientific programme has been extensively carried out was the electron-Volt Spectrometer (eVS) at the ISIS neutron source, where the first experimental determinations of the momentum distribution and mean kinetic energy were carried out on hydrogen containing systems [214-216].

The key factors that allowed to enhance the performances of this type of spectrometer were directed towards a significant improvement on the resolution and on count rate capabilities. These involved either new methods of energy selection (Double Difference $[95,94,93]$ and Foil Cycling $[110,111]$ ), as well as detection concepts such as the Resonant Detector $[17,20$, 184,31]. On Vesuvio, in the current configuration, the use of YAP detectors in conjunction with the Foil Cycling technique in the forward scattering bank, allows a momentum space resolution for proton momentum distribution studies of $\frac{\Delta p}{p}=13 \%$, and counting statistics errors of $1 \%$ at the centre of the overall Neutron Compton profile and of $15 \%$ at $1 / 15$ of the peak height, respectively. In this report we have presented a description of the detection techniques and methodologies that have been developed for $\mathrm{eV}$ spectrometers, and that demonstrated their potential for studies of condense matter, together with the perspective on the research activity that can be devoted to subsequent developments on eV instrumentation. The main body of research presented here started in the last decade, with the most significant advances carried out since 
2005. The experimental studies that followed, have stimulated new theoretical approaches on nuclear quantum effects in hydrogen-containing systems [164,26,165-167] and may prove a benchmark test of new ab-initio methods, such as the coloured-noise approach to the quantum description of molecular systems [166]. These represent a step beyond the state of the art described in the latest review on momentum distribution studies using inelastic neutron scattering of Ref. [41]. It is interesting to recall what was envisaged in terms of perspectives of the technique: "...we expect the window into the quantum reality provided by the exploitation of high-energy neutrons to provide a unique perspective on systems of interest to a wide range of disciplines. And, as with any other new window, we expect that much of what we will find most interesting in these investigations will be unexpected...". These expectations were not only confirmed, but were surpassed, as for example in the case of the extension of this spectroscopic technique to proteins [217,218] and nucleic acids [140], or cultural heritage [189, 219]. We feel in turn obliged to formulate a prospect for the future: the development of instrumentation and methods, some of which we have outlined here, will allow at extending this kind of studies, for example momentum distribution of heavier mass systems to understand the influence of chemical environment on the dynamics of deuterium, lithium, oxygen, up to sodium. Moreover, the optimization of low angle detector banks, making use of the experimental findings on directional components of the gamma background (see Section 2), coupled with Foil Cycling technique, may allow a simultaneous measurement of single-particle excitations (e.g. vibrational spectrum around the stretching mode [88] and/or electronic transitions) and momentum distribution, enhancing the scope of eV neutron spectroscopy. Finally, the aim of this report is to provide a detailed reference for $\mathrm{eV}$ neutron spectrometers in view of the possibility opened to the construction of this class of instruments of unprecedented performance at the next generation spallation sources worldwide [220-222].

\section{Acknowledgments}

This work was supported within the CNR-CCLRC Agreement No. 01/9001 concerning collaboration in scientific research at the spallation neutron source ISIS. The financial support of the Consiglio Nazionale delle Ricerche in this research is hereby acknowledged. AP acknowledges the CNISM-CNR joint research program. The authors would like to thank J.M. Carpenter for stimulating the writing of the manuscript. We feel indebted to C. Andreani, G. Gorini for their guidance, and M. Tardocchi, E. Perelli Cippo, J. Mayers, M. Adams, G. Reiter, N.J. Rhodes, E. M. Schooneveld, for the fruitful time spent together in the study of methods, devices and techniques for electron volt neutron spectroscopy.

\section{References}

[1] R. Bedogni, A. Esposito, C. Andreani, R. Senesi, M.P. de Pascale, P. Picozza, A. Pietropaolo, G. Gorini, C.D. Frost, S. Ansell, Characterization of the neutron field at the ISIS-VESUVIO facility by means of a bonner sphere spectrometer, Nuclear Instruments and Methods in Physics Research A 612 (2009) 143-148.

[2] M. Harada, N. Watanabe, F. Maekawa, M. Futakawa, Pulse characteristics of epithermal neutrons from a spallation source, Nuclear Instruments and Methods in Physics Research A 597 (2008) 242-256.

[3] J. Carpenter, T. Mason, A long-wavelength target station for the spallation neutron source, Nuclear Instruments and Methods in Physics Research Section A: Accelerators, Spectrometers, Detectors and Associated Equipment 545 (1-2) (2005) 1-19. URL: http://www.sciencedirect.com/science/ article/B6TJM-4G0JWXP-1/2/9ed13b8f7daa84b559ec747bf94371c2.

[4] M. Flaska, A. Borella, D. Lathouwers, L.C. Mihailescu, W. Mondelaers, A.J.M. Plompen, H. van Dam, T.H.J.J. van der Hagen, Modeling of the GELINA neutron target using coupled electron-photon-neutron transport with the MCNP4C3 code, Nuclear Instruments and Methods in Physics Research A 531 (2004) 392-406.

[5] P.E. Koehler, Measurement of the lansce neutron flux from 0.025 ev to $100 \mathrm{Kev}$, Nuclear Instruments and Methods in Physics Research Section A: Accelerators, Spectrometers, Detectors and Associated Equipment 292 (3) (1990) 541-545. URL: http://www.sciencedirect.com/science/article/B6TJM470F9WH-3P/2/8f99cebb806419d4d15536d20af43d03.

[6] R. Senesi, C. Andreani, Z. Bowden, D. Colognesi, E. Degiorgi, A.L. Fielding, J. Mayers, M. Nardone, J. Norris, M. Praitano, N.J. Rhodes, W.G. Stirling, J. Tomkinson, C. Uden, VESUVIO: a novel instrument for performing spectroscopic studies in condensed matter with eV neutrons at the ISIS facility, Physica B (Condensed Matter) 276 (2000) 200-201.

[7] P. Bisanti, S.W. Lovesey, Condensed matter research using the spallation neutron source isis, Physica Scripta 1987 (T19A) (1987) 47. URL: http://stacks.iop.org/1402-4896/1987/i=T19A/a=007.

[8] M.T.F. Telling, K.H. Andersen, Spectroscopic characteristics of the OSIRIS near-backscattering crystal analyser spectrometer on the ISIS pulsed neutron source, Physical Chemistry Chemical Physics (Incorporating Faraday Transactions) 7 (2005) 1255-+.

[9] J.M. Carpenter, T.H. Blewitt, D.L. Price, S.A. Werner, Pulsed spallation neutron sources, Physics Today 32 (12) (1979) 42-49. URL: http://link.aip.org/link/?PTO/32/42/1.

[10] V.I. Gol'danskii, Soviet Journal of Experimental and Theoretical Physics 4 (1957) 604

[11] G.K. Ivanov, Y.S. Sayasov, Theory of the vibrational excitation of a molecule in the impulse approximation, Soviet Physics Doklady 9 (1964) 171-+.

[12] P.C. Hohenberg, P.M. Platzman, High-Energy Neutron Scattering from Liquid He4 , Physical Review 152 (1966) 198-200.

[13] G.K. Ivanov, Y.S. Sayasov, Reviews of topical problems: interaction of neutrons with molecules, Soviet Physics Uspekhi 9 (1967) 670-691.

[14] R.N. Sinclair, M.C. Moxon, J.M. Carpenter, Bullettin of the American Physical Society 22 (1977) 101.

[15] L. Cser, N. Kroo, P. Pacher, V. Simkin, E. Vasilyeva, Epithermal inelastic neutron spectrometry using a resonance detector, Nuclear Instruments and Methods 179 (1981) 515-518.

[16] R.G. Johnson, C.D. Bowman, Inelastic-scattering measurements of 1.5-15 eV neutrons, Physical Review Letters 49 (1982) 797-800.

[17] J.M. Carpenter, N. Watanabe, S. Ikeda, Y. Masuda, S. Sato, Tests of a resonance detector spectrometer for electron-volt spectroscopy, in: Proceedings of the Sixth International Collaboration on Advanced Neutron Sources, 1982, pp. 266-278.

[18] J. Carpenter, N. Watanabe, S. Ikeda, Y. Masuda, S. Sato, A Resonance Detector Spectrometer at Kens, Physica B vol. 120B+C, Netherlands, $1983 / 05$. pp. $\quad 126-30, \quad$ bi $\langle$ sub $\rangle 12\langle/$ sub $\rangle \mathrm{GeO}\langle$ sub $\rangle 20\langle/$ sub $\rangle$ scintillators; $\langle$ sup $\rangle 181\langle/$ sup $\rangle$ Taresonance; $\langle$ sup $\rangle 121\langle/$ sup $\rangle$ Sbresonance; $\langle$ sup $\rangle 149\langle/$ sup $\rangle$ Sm resonance; $\mathrm{Bi}$; $\mathrm{V}$; resonance detector spectrometer; KENS neutron source; time-focusing principle; graphite; cooled absorbers;. URL: http://dx.doi.org/10.1016/0378-4363(83)90354-6.

[19] J. Carpenter, N. Watanabe, Time focussing and resolution in resonance detector neutron spectrometers, Nuclear Instruments and Methods in Physics Research $213(2-3)$ (1983) 311-316. URL: http://www.sciencedirect.com/science/article/B73DP-470WG0K-78/2/ de7b6b2b5080f2569251837ea0ab6679. 
[20] R.K. Crawford, The IPNS resonance detector spectrometer, in: Proceedings of the Ninth International Collaboration on Advanced Neutron Sources, 1986, pp. 397-430.

[21] G.C. Windsor, Pulsed Neutron Scattering, Taylor \% Francis Ltd., London, 1981.

[22] G.S. Samosvat, Y.S. Sayasov, V.T. Chuburkov, Scattering of slow neutrons by water molecules, Soviet Journal of Experimental and Theoretical Physics 27 (1968) $15-+$.

[23] R.N. Silver, P.E. Sokol, Momentum Distributions, Plenum, New York, 1989.

[24] D. Homouz, G. Reiter, J. Eckert, J. Mayers, R. Blinc, Measurement of the 3D Born-Oppenheimer potential of a proton in a hydrogen-bonded system via deep inelastic neutron scattering: the superprotonic conductor $\mathrm{Rb}_{3} \mathrm{H}\left(\mathrm{SO}_{4}\right)_{2}$, Physical Review Letters 98 (11) (2007) 115502-+.

[25] G. Reiter, A. Shukla, P.M. Platzman, J. Mayers, Deuteron momentum distribution in $\mathrm{KD}_{2} \mathrm{PO}_{4}$, New Journal of Physics 10 (1) (2008) $013016-+$.

[26] L. Lin, J.A. Morrone, R. Car, M. Parrinello, Displaced path integral formulation for the momentum distribution of quantum particles, Physical Review Letters 105 (11) (2010) 110602.

[27] C. Stock, R.A. Cowley, J.W. Taylor, S.M. Bennington, High-energy neutron scattering from hydrogen using a direct geometry spectrometer, Physical Review B 81 (2) (2010) 024303-+.

[28] A. Taylor, Low-Q inelastic scattering with eV neutrons, Nuclear Instruments and Methods in Physics Research 224 (1984) 133-141.

[29] S.K. Sinha, The uses of pulsed neutron sources for research in magnetism and other areas of condensed matter physics, Journal of Applied Physics 50 (1979) 1952-1957.

[30] E. Perelli Cippo, G. Gorini, M. Tardocchi, A. Pietropaolo, C. Andreani, R. Senesi, N.J. Rhodes, E.M. Schooneveld, The very low angle detector for highenergy inelastic neutron scattering on the VESUVIO spectrometer, Nuclear Instruments and Methods in Physics Research A 589 (2008) $296-303$.

[31] C. Andreani, A. Pietropaolo, R. Senesi, G. Gorini, E. Perelli-Cippo, M. Tardocchi, N. Rhodes, E.M. Schooneveld, A resonant detector for high-energy inelastic neutron scattering experiments, Applied Physics Letters 85 (2004) 5454-+.

[32] H. Postma, M. Blaauw, P. Bode, P. Mutti, F. Corvi, P. Siegler, Neutron-resonance capture analysis of materials, Journal of Radioanalytical and Nuclear Chemistry 248 (2001) 115-120. doi:10.1023/A:1010690428025. URL: http://dx.doi.org/10.1023/A:1010690428025.

[33] M. Blaauw, H. Postma, P. Mutti, An attempt to date an antique benin bronze using neutron resonance capture analysis, Applied Radiation and Isotopes 62 (3) (2005) 429-433. URL:http://www.sciencedirect.com/science/article/B6TJ0-4DCWCTP-8/2/3174bc7bbe15db2eed4bddcb1a10d0da.

[34] E. Perelli Cippo, A. Borella, G. Gorini, W. Kockelmann, A. Pietropaolo, H. Postma, N.J. Rhodes, P. Schillebeeckx, E.M. Schooneveld, M. Tardocchi, R. Wynants, ANCIENT CHARM Collaboration, A detector system for neutron resonance capture imaging, Nuclear Instruments and Methods in Physics Research A 623 (2010) 693-698.

[35] B.N. Brockhouse, D.G. Hurst, Energy distribution of slow neutrons scattered from solids, Physical Review 88 (1952) 542-547.

[36] B.N. Brockhouse, A.T. Stewart, Scattering of neutrons by phonons in an aluminum single crystal, Physical Review 100 (1955) $756-757$.

[37] R. Lowde, The principles of mechanical neutron-velocity selection, Journal of Nuclear Energy. Part A. Reactor Science 11 (2-4) (1960) 69-80.

[38] R. Bewley, Enhancing the flux on a chopper spectrometer with a dual beam compression monochromator, Nuclear Instruments and Methods in Physics Research, Section A: Accelerators, Spectrometers, Detectors and Associated Equipment 492 (1-2) (2002) 97-104.

[39] G.F. Knoll, Radiation Detection and Measurement, J. Wiley \& Sons, New York, 1979.

[40] G.I. Watson, Journal of Physics: Condensed Matter 8 (1996) 5955.

[41] C. Andreani, D. Colognesi, J. Mayers, G.F. Reiter, R. Senesi, Advances in Physics 54 (2005) 377.

[42] P.R. O'Connor, G.T. Seaborg, High energy spallation and fission products of uranium, Physical Review 74 (1948) 1189-1190.

[43] M. Enke, C. Herbach, D. Hilscher, U. Jahnke, O. Schapiro, A. Letourneau, J. Galin, F. Goldenbaum, B. Lott, A. Péghaire, D. Filges, R. Neef, K. Nünighoff, N. Paul, H. Schaal, G. Sterzenbach, A. Tietze, L. Pienkowski, Evolution of a spallation reaction: experiment and Monte Carlo simulation, Nuclear Physics A 657 (1999) 317-339.

[44] N. Watanabe, Neutronics of pulsed spallation neutron sources, Reports on Progress in Physics 66 (2003) 339-381.

[45] J. Carpenter, Pulsed spallation neutron sources for slow neutron scattering, Nuclear Instruments and Methods 145 (1977) 91-113.

[46] S. Leray, F. Borne, S. Crespin, J. Fréhaut, X. Ledoux, E. Martinez, Y. Patin, E. Petibon, P. Pras, A. Boudard, R. Legrain, Y. Terrien, F. Brochard, D. Drake, J.C. Duchazeaubeneix, J.M. Durand, S.I. Meigo, G. Milleret, D.M. Whittal, W. Wlazlo, D. Durand, C. Le brun, F.R. Lecolley, J.F. Lecolley, F. Lefebvres, M. Louvel, C. Varignon, F. Hanappe, S. Ménard, L. Stuttge, J. Thun, Spallation neutron production by 0.8, 1.2, and $1.6 \mathrm{GeV}$ protons on various targets, Physical Review B 65 (4) (2002) 044621-+.

[47] J.D. Jackson, Classical Electrodynamics, John Wiley \& Sons Inc., New York, 1998.

[48] G.C. Baldwin, G.S. Klaiber, X-ray yield curves for $\gamma-n$ reactions, Physical Review 73 (10) (1948) 1156-1163.

[49] D.H. Day, R.N. Sinclair, Neutron moderator assemblies for pulsed thermal neutron time-of-flight experiments, Nuclear Instruments and Methods 72 (1969) 237-+.

[50] M. Flaska, A. Borella, D. Lathouwers, L.C. Mihailescu, W. Mondelaers, A.J.M. Plompen, H. van Dam, T.H.J.J. van der Hagen, Modeling of the GELINA neutron target using coupled electron-photon-neutron transport with the MCNP4C3 code, Nuclear Instruments and Methods in Physics Research A 531 (2004) 392-406.

[51] A.M. Weinberg, P.E. Wigner, The Theory of Neutron Chain Reactor, Univ. of Chicago Press, Chicago, 1958.

[52] B.G. Harwey, Progress in Nuclear Physics, Pergamon Press, New York, 1959.

[53] R. Serber, Nuclear reactions at high energies, Physical Review 72 (11) (1947) 1114-1115.

[54] V. Weisskopf, Statistics and nuclear reactions, Physical Review 52 (4) (1937) 295-303.

[55] L. Pienkowski, H.G. Bohlen, J. Cugnon, H. Fuchs, J. Galin, B. Gatty, B. Gebauer, D. Guerreau, D. Hilscher, D. Jacquet, U. Jahnke, M. Josset, X. Ledoux, S. Leray, B. Lott, M. Morjean, A. Pghaire, G. Rschert, H. Rossner, R.H. Siemssen, C. Stéphan, Physics Letters B 336 (2) (1994) 147-151.

[56] X. Ledoux, H.G. Bohlen, J. Cugnon, H. Fuchs, J. Galin, B. Gatty, B. Gebauer, D. Guerreau, D. Hilscher, D. Jacquet, U. Jahnke, M. Josset, S. Leray, B. Lott, M. Morjean, B.M. Quednau, G. Röschert, H. Rossner, A. Péghaire, L. Pienkowski, R.H. Siemssen, C. Stéphan, Physical Review, C 57 (5) (1998) $2375-2392$.

[57] M. Enke, C.M. Herbach, D. Hilscher, U. Jahnke, O. Schapiro, A. Letourneau, J. Galin, F. Goldenbaum, B. Lott, A. Pghaire, D. Filges, R.D. Neef, K. Nnighoff, N. Paul, H. Schaal, G. Sterzenbach, A. Tietze, L. Pienkowski, Nuclear Physics, A 657 (3) (1999) 317-339.

[58] A. Pietropaolo, M. Tardocchi, E.M. Schooneveld, R. Senesi, Characterization of the $\gamma$ background in epithermal neutron scattering measurements at pulsed neutron sources, Nuclear Instruments and Methods in Physics Research A 568 (2006) 826-838.

[59] A. Pietropaolo, E. Perelli Cippo, G. Gorini, M. Tardocchi, E.M. Schooneveld, C. Andreani, R. Senesi, $\gamma$-ray background sources in the VESUVIO spectrometer at ISIS spallation neutron source, Nuclear Instruments and Methods in Physics Research A 608 (2009) 121-124.

[60] M. Tardocchi, G. Gorini, A. Pietropaolo, C. Andreani, R. Senesi, N. Rhodes, E.M. Schooneveld, YAP scintillators for resonant detection of epithermal neutrons at pulsed neutron sources, Review of Scientific Instruments 75 (2004) 4880-4890.

[61] S.F. Mughabghab, Neutron Cross Sections, Academic Press, Orlando, 1984.

[62] T.F. Koetzle, P.M.B. Piccoli, A.J. Schultz, Single-crystal neutron diffraction studies of hydrogen-bonded systems: two recent examples from IPNS, Nuclear Instruments and Methods in Physics Research A 600 (2009) 260-262.

[63] A. Daoud-Aladine, B. Kundys, C. Martin, P.G. Radaelli, P.J. Brown, C. Simon, L.C. Chapon, Multiferroicity and spiral magnetism in FeVO4 with quenched Fe orbital moments, Physical Review B 80 (22)(2009).

[64] K. Matan, S. Ibuka, R. Morinaga, S. Chi, J.W. Lynn, A.D. Christianson, M.D. Lumsden, T.J. Sato, Doping dependence of spin dynamics in electron-doped $\mathrm{Ba}(\mathrm{Fe} 1-\mathrm{xCox})(2) \mathrm{As}-2$, Physical Review B 82 (5) (2010).

[65] D. Reznik, J.P. Ismer, I. Eremin, L. Pintschovius, T. Wolf, M. Arai, Y. Endoh, T. Masui, S. Tajima, Local-moment fluctuations in the optimally doped high-T-c superconductor YBa2Cu306.95, Physical Review B 78 (13) (2008).

[66] J.M. Carpenter, G.H. Lander, C.G. Windsor, Instrumentation at pulsed neutron sources, Review of Scientific Instruments 55 (1984) $1019-1043$. 
[67] R.M. Brugger, A.D. Taylor, C.E. Olsen, J.A. Goldstone, A.K. Soper, A spectrometer for inelastic scattering using neutrons from 1 to 186 ev, Nuclear Instruments and Methods in Physics Research 221 (2)(1984) 393-407. URL:http://www.sciencedirect.com/science/article/B73DP-478HFR220/2/c56fd981fa54c58efa2c6d5177da312c.

[68] D. Colognesi, M. Celli, F. Cilloco, R. Newport, S. Parker, V. Rossi-Albertini, F. Sacchetti, J. Tomkinson, M. Zoppi, TOSCA neutron spectrometer: the final configuration, Applied Physics A-Materials Science \& Processing 74 (Part 1 Suppl. S) (2002) S64-S66.

[69] P. Georgiev, D. Ross, A.De. Monte, U. Montaretto-Marullo, R. Edwards, A. Ramirez-Cuesta, M. Adams, D. Colognesi, In situ inelastic neutron scattering studies of the rotational and translational dynamics of molecular hydrogen adsorbed in single-wall carbon nanotubes (SWNTs), Carbon 43 (5) (2005) 895-906.

[70] S.O. Diallo, R.T. Azuah, O. Kirichek, J.W. Taylor, H.R. Glyde, Limits on bose-einstein condensation in confined solid ${ }^{4}$ He, Physical Review B 80 (6) (2009) 060504.

[71] S.E. Nagler, D.A. Tennant, R.A. Cowley, T.G. Perring, S.K. Satija, Spin dynamics in the quantum antiferromagnetic chain compound KCuF 3 , Physical Review B 44 (22) (1991) 12361-12368.

[72] C. Stock, R.A. Cowley, J.W. Taylor, S.M. Bennington, High energy neutron scattering from hydrogen using a direct geometry spectrometer (2009). URL: http://www.citebase.org/abstract?id=oai:arXiv.org:0907.1945.

[73] J. Mayers, A.C. Evans, Momentum distributions in fluids determined by neutron compton scattering, Nuovo Cimento D Serie 16 (1994) 737-745.

[74] R.G. Johnson, Detectors for neutron scattering in the eV region, Nuclear Instruments and Methods in Physics Research A 263 (1988) $427-435$.

[75] J.M. Carpenter, N. Watanabe, S. Ikeda, Y. Masuda, S. Sato, Resonance detector spectrometer at kens, Physica B: Physics of Condensed Matter 120 (1-3) (1982) 126-130.

[76] H. Rauh, N. Watanabe, Determination of the momentum distribution of scattering particles from high-Q scattering spectra of a resonance detector neutron spectrometer, Nuclear Instruments and Methods in Physics Research A 228 (1984) 147-158.

[77] Y. Nakai, E. Akiba, H. Asano, S. Ikeda, Neutron compton scattering with an eV neutron spectrometer, Journal of the Physical Society of Japan 61 (1992) 1834-+.

[78] S. Ikeda, N. Watanabe, Neutron scattering from superfluid ${ }^{4}$ He at very large momentum transfer, Physics Letters A 121 (1) (1987) 34-38.

[79] R. Senesi, A. Pietropaolo, C. Andreani, Constant-q data representation in neutron compton scattering on the VESUVIO spectrometer, Nuclear Instruments and Methods in Physics Research A 594 (2008) 244-252.

[80] M. Harada, N. Watanabe, F. Maekawa, M. Futakawa, Pulse characteristics of epithermal neutrons from a spallation source, Nuclear Instruments \& Methods in Physics Research Section A-Accelerators Spectrometers Detectors and Associated Equipment 597 (2-3) (2008) $242-256$.

[81] S. Imberti, C. Andreani, V. Garbuio, G. Gorini, A. Pietropaolo, R. Senesi, M. Tardocchi, Nuclear Instruments and Methods in Physics Research A 522 (2005) 463

[82] D.R. Allen, E.W.J. Mitchell, R.N. Sinclair, A resonance detector spectrometer for neutron inelastic scattering in the eV region, Journal of Physics E Scientific Instruments 13 (1980) 639-646.

[83] S. Ikeda, J.M. Carpenter, Wide-energy-range, high-resolution measurements of neutron pulse shapes of polyethylene moderators, Nuclear Instruments and Methods in Physics Research A 239 (1985) 536-544.

[84] C. Andreani, G. Baciocco, R.S. Holt, J. Mayers, Resolution in deep inelastic neutron scattering using pulsed neutron sources, Nuclear Instruments and Methods in Physics Research A 276 (1989) 297-305.

[85] R. Senesi, C. Andreani, D. Colognesi, Momentum distribution of liquid ${ }^{3}$ He: simulation of deep inelastic neutron scattering experiments with the VESUVIO spectrometer, Journal of Low Temperature Physics 126 (2002) 57-62.

[86] C. Andreani, P. Bosi, F. Sacchetti, C.K. Loong, Absolute measurements of the stretching mode density of states in polycrystalline ice Ih, Journal of Chemical Physics 83 (1985) 750-753.

[87] C. Andreani, A. Pietropaolo, R. Senesi, G. Gorini, E. Perelli-Cippo, M. Tardocchi, N. Rhodes, E.M. Schooneveld, A resonant detector for high-energy inelastic neutron scattering experiments, Applied Physics Letters 85 (2004) 5454-+.

[88] E. Perelli-Cippo, G. Gorini, M. Tardocchi, C. Andreani, A. Pietropaolo, R. Senesi, N.J. Rhodes, E.M. Schooneveld, The O H stretching band in ice Ih derived via eV neutron spectroscopy on VESUVIO using the new very low angle detector bank, Applied Physics A: Materials Science \& Processing 83 (2006) 453-460.

[89] P. Novák, M. Diviš, Crystal field parameters of praseodymium in oxides, Physica Status Solidi B Basic Research 244 (2007) $3168-3177$.

[90] A.D. Taylor, R. Osborn, K.A. Mcewen, W.G. Stirling, Z.A. Bowden, W.G. Williams, E. Balcar, S.W. Lovesey, Intermultiplet transitions in praseodymium using neutron spectroscopy, Physical Review Letters 61 (1988) 1309-1312.

[91] R. Osborn, High energy magnetic inelastic neutron scattering at ISIS, Physica B (Condensed Matter) 159 (1989) $151-160$.

[92] R. Senesi, D. Colognesi, A. Pietropaolo, T. Abdul-Redah, Deep inelastic neutron scattering from orthorhombic ordered HCl: short-time proton dynamics and anomalous neutron cross sections, Physical Review B 72 (5) (2005) 054119-+.

[93] J. Mayers, J. Tomkinson, T. Abdul-Redah, W.G. Stirling, C. Andreani, R. Senesi, M. Nardone, D. Colognesi, E. Degiorgi, VESUVIO-the double difference inverse geometry spectrometer at ISIS, Physica B (Condensed Matter) 350 (2004) E659-E662.

[94] C. Andreani, D. Colognesi, E. Degiorgi, A. Filabozzi, M. Nardone, E. Pace, A. Pietropaolo, R. Senesi, Double difference method in deep inelastic neutron scattering on the VESUVIO spectrometer, Nuclear Instruments and Methods in Physics Research A 497 (2003) 535-549.

[95] P.A. Seeger, A.D. Taylor, R.M. Brugger, Nuclear Instruments and Methods in Physics Research A 240 (1985) $98-114$.

[96] H. Rauh, N. Watanabe, Detector resolution in a resonance detector neutron spectrometer, Nuclear Instruments and Methods in Physics Research 222 (3) (1984) 507-516. URL:http://www.sciencedirect.com/science/article/B73DP-478JGBV-J/2/d5c6bddda1a0d7c0627a59ed6477fee6.

[97] A. Pietropaolo, C. Andreani, A. D’Angelo, R. Senesi, G. Gorini, S. Imberti, M. Tardocchi, N. Rhodes, E.S. Schooneveld, $\gamma$ detectors for deep inelastic neutron scattering in the 1-100 eV energy region, Applied Physics A: Materials Science \& Processing 74 (2002) 189-190.

[98] C. Andreani, A. Pietropaolo, R. Senesi, G. Gorini, M. Tardocchi, A. Bracco, N. Rhodes, E. Schooneveld, Electron-volt spectroscopy at a pulsed neutron source using a resonance detector technique, Nuclear Instruments and Methods in Physics Research A 481 (2002) 509-520.

[99] M. Tardocchi, A. Pietropaolo, C. Andreani, A. Bracco, A. D’angelo, G. Gorini, S. Imberti, R. Senesi, N.J. Rhodes, E.M. Schooneveld, Cadmium-Zinc-Telluride photon detector for epithermal neutron spectroscopy-pulse height response characterisation, Nuclear Instruments and Methods in Physics Research A 526 (2004) 477-492.

[100] P.B. Johnson, J.M.G. Caraça, J.N. Pihl, R.D. Gill, H.J. Rose, An associated gamma ray time of flight spectrometer with high neutron efficiency, Nuclear Instruments and Methods 93 (1971) 417-+.

[101] G. Breit, E. Wigner, Capture of slow neutrons, Physical Review 49 (7) (1936) 519-531.

[102] W.E. Lamb, Capture of neutrons by atoms in a crystal, Physical Review 55 (2) (1939) 190-197.

[103] M.S. Nelkin, D.E. Parks, Effects of chemical binding on nuclear recoil, Physical Review 119 (3) (1960) $1060-1068$.

[104] F. Rahn, H.S. Camarda, G. Hacken, W.W. Havens, H.I. Liou, J. Rainwater, M. Slagowitz, S. Wynchank, Neutron resonance spectroscopy. X. ${ }^{232}$ Th and ${ }^{238}$ U, Physical Review C 6 (1972) 1854-1869.

[105] A.A. El Kady, D. Duffey, P.F. Wiggins, Gamma rays from thermal neutron capture in gold, Zeitschrift fur Physik 245 (1971) $198-203$.

[106] P. Fougeres, P. Siffert, M. Hageali, J.M. Koebel, R. Regal, CdTe and $\mathrm{Cd}_{1-x} \mathrm{Zn}_{x}$ Te for nuclear detectors: facts and fictions, Nuclear Instruments and Methods in Physics Research A 428 (1999) 38-44.

[107] C.E. Porter, R.G. Thomas, Fluctuations of nuclear reaction widths, Physical Review 104 (2) (1956) $483-491$.

[108] H.I. Liou, R.E. Chrien, Nuclear Science and Engineering 62 (1977) 463. 
[109] M. Chadwick, P. Oblozinsk, M. Herman, N. Greene, R. Mcknight, D. Smith, P. Young, R. Macfarlane, G. Hale, S. Frankle, A. Kahler, T. Kawano, R. Little, D. Madland, P. Moller, R. Mosteller, P. Page, P. Talou, H. Trellue, M. White, W. Wilson, R. Arcilla, C. Dunford, S. Mughabghab, B. Pritychenko, D. Rochman, A. Sonzogni, C. Lubitz, T. Trumbull, J. Weinman, D. Brown, D. Cullen, D. Heinrichs, D. Mcnabb, H. Derrien, M. Dunn, N. Larson, L. Leal A. Carlson, R. Block, J. Briggs, E. Cheng, H. Huria, M. Zerkle, K. Kozier, A. Courcelle, V. Pronyaev, S. van der Marck, Endf/b-vii.0: next generation evaluated nuclear data library for nuclear science and technology, Nuclear Data Sheets 107 (12) (2006) 2931-3060, evaluated Nuclear Data File ENDF/B-VII.0. URL: http://www.sciencedirect.com/science/article/B6WNV-4MGDW8W-1/2/5040b3d5640d1c334634e9f83c754893.

[110] E.M. Schooneveld, J. Mayers, N.J. Rhodes, A. Pietropaolo, C. Andreani, R. Senesi, G. Gorini, E. Perelli-Cippo, M. Tardocchi, Foil cycling technique for the VESUVIO spectrometer operating in the resonance detector configuration, Review of Scientific Instruments 77 (2006) $5103-+$

[111] A. Pietropaolo, C. Andreani, A. Filabozzi, E. Pace, R. Senesi, Resolution function in deep inelastic neutron scattering using the foil cycling technique, Nuclear Instruments \& Methods in Physics Research, Section A (Accelerators, Spectrometers, Detectors and Associated Equipment) 570 (2007) 498-510.

[112] J.M. Carpenter, Pulsed spallation neutron sources for slow neutron scattering, Nuclear Instruments and Methods 145 (1) (1977) 91-113. URL: http://www.sciencedirect.com/science/article/B73DN-471XVHY-298/2/cf80c2b616310b46a45b7d1a5b9f571e.

[113] M. Harada, N. Watanabe, M. Teshigawara, T. Kai, T. Kato, Y. Ikeda, Neutronics of a poisoned para-hydrogen moderator for a pulsed spallation neutron source, Nuclear Instruments and Methods in Physics Research A 574 (2007) 407-419.

[114] S.J. Friesenhahn, M.P. Fricke, D.G. Costello, W.M. Lopez, A.D. Carlson, Neutron resonance parameters and radiative capture cross section of Gd from $3 \mathrm{eV}$ to $750 \mathrm{keV}$, Nuclear Physics A 146 (1970) 337-358.

[115] A. Pietropaolo, C. Andreani, A. Filabozzi, R. Senesi, G. Gorini, E. Perelli-Cippo, M. Tardocchi, N.J. Rhodes, E.M. Schooneveld, Dins measurements on vesuvio in the resonance detector configuration: proton mean kinetic energy in water, Journal of Instrumentation 1 (04) (2006) P04001. URL:http://stacks.iop.org/1748-0221/1/i=04/a=P04001.

[116] G. Gorini, E. Perelli-Cippo, M. Tardocchi, C. Andreani, A. D’Angelo, A. Pietropaolo, R. Senesi, S. Imberti, A. Bracco, E. Previtali, G. Pessina, N.J. Rhodes, E.M. Schooneveld, The resonant detector and its application to epithermal neutron spectroscopy, Nuclear Instruments and Methods in Physics Research A 529 (2004) 293-300.

[117] V.F. Turkin, Slow Neutrons, Israel Program for Scientific Translations, Jerusalem, 1965.

[118] G.B. West, Electron scattering from atoms, nuclei and nucleons, Physics Reports 18 (1975) 263-323.

[119] S.W. Lovesey, The Theory Of Neutron Scattering From Condensed Matter, Oxford University Press, Oxford, 1986.

[120] R. Scherm, Fundamentals of neutron scattering by condensed matter, Annales de Physique 7 (5) (1972) 349-370.

[121] D.F. McMorrow, R.A. Cowley, R.M. Nicklow, P.W. Mitchell, A.D. Taylor, M. Mostoller, A neutron scattering study of the impulse approximation in single-crystal beryllium, Journal of Physics Condensed Matter 2 (1990) 1045-1057.

[122] H.A. Gersch, L.J. Rodriguez, P.N. Smith, Corrections to the impulse approximation for high-energy neutron scattering from liquid helium, Physica Review A 5 (1972) 1547-1558.

[123] V.F. Sears, Scaling and final-state interactions in deep-inelastic neutron scattering, Physical Review B 30 (1984) 44-51.

[124] R.N. Silver, G. Reiter, Structure dependence of final-state effects in deep inelastic neutron scattering: quasiclassical theory, Physical Review B 35 (1987) 3647-3650

[125] R.N. Silver, Theory of neutron scattering experiments on momentum distributions in quantum fluids, Continuum Mechanics and Thermodynamics (1987), Los Alamos Technical report LA-UR-87-2534; CONF-8707110-1.

[126] A. Pietropaolo, R. Senesi, C. Andreani, J. Mayers, Quantum effects in water: proton kinetic energy maxima in stable and supercooled liquid, Brazilian Journal of Physics 39 (2009) 318-321.

[127] R. Moreh, D. Nemirovsky, On the proton kinetic energy in $\mathrm{H}_{2} \mathrm{O}$ and in nanotube water, Journal of Chemical Physics 133 (8) (2010) $084506-+$

[128] A.K. Soper, Comment on "Excess of proton mean kinetic energy in supercooled water", Physical Review Letters 103 (6) (2009) 069801-+.

[129] D. Flammini, M.A. Ricci, F. Bruni, A new water anomaly: the temperature dependence of the proton mean kinetic energy, Journal of Chemical Physics 130 (23) (2009) 236101-+.

[130] A. Pietropaolo, R. Senesi, C. Andreani, A. Botti, M.A. Ricci, F. Bruni, Pietropaolo et al. reply, Physical Review Letters 103 (6) (2009) 069802-+.

[131] A. Pietropaolo, R. Senesi, C. Andreani, A. Botti, M.A. Ricci, F. Bruni, Excess of proton mean kinetic energy in supercooled water, Physical Review Letters 100 (12) (2008) 127802-+.

[132] C. Pantalei, A. Pietropaolo, R. Senesi, S. Imberti, C. Andreani, J. Mayers, C. Burnham, G. Reiter, Proton momentum distribution of liquid water from room temperature to the supercritical phase, Physical Review Letters 100 (17) (2008) 177801-+.

[133] G.F. Reiter, J.C. Li, J. Mayers, T. Abdul-Redah, P. Platzman, The proton momentum distribution in water and ice, Brazilian Journal of Physics 34 (2004) $142-147$.

[134] C. Andreani, D. Colognesi, E. Degiorgi, M.A. Ricci, Proton dynamics in supercritical water, Journal of Chemical Physics 115 (2001) $11243-11248$.

[135] C. Andreani, E. Degiorgi, R. Senesi, F. Cilloco, D. Colognesi, J. Mayers, M. Nardone, E. Pace, Single particle dynamics in fluid and solid hydrogen sulphide: an inelastic neutron scattering study, Journal of Chemical Physics 114 (2001) 387-398.

[136] H. Yokoyama, M. Kannami, H. Kanno, Existence of clathrate-like structures in supercooled water: X-ray diffraction evidence, Chemical Physics Letters 463 (2008) 99-102.

[137] G.F. Reiter, J. Mayers, P. Platzman, Direct observation of tunneling in KDP using neutron compton scattering, Physical Review Letters 89 (13) (2002) 135505-+.

[138] A. Pietropaolo, D. Fernandez-Cañoto, E. Perelli-Cippo, S. Dirè, P. Prosposito, Subfemtosecond dynamics of structural protons in silica xerogels, Physical Review B 77 (1) (2008) 014202-+

[139] C. Pantalei, R. Senesi, C. Andreani, P. Sozzani, A. Comotti, S. Bracco, M. Beretta, P.E. Sokol, G. Reiter, Interaction of single water molecules with silanols in mesoporous silica, Physical Chemistry Chemical Physics (Incorporating Faraday Transactions) 13 (2011) 6022-+.

[140] G.F. Reiter, R. Senesi, J. Mayers, Changes in the zero-point energy of the protons as the source of the binding energy of water to A-phase DNA, Physical Review Letters 105 (14) (2010) 148101-+.

[141] H.R. Glyde, Excitations in Liquid and Solid Helium, Clarendon, Oxford, 1994.

[142] E.R. Dobbs, Helium Three, Oxford University Press, Oxford, 2000.

[143] S.O. Diallo, J.V. Pearce, R.T. Azuah, H.R. Glyde, Physical Review Letters 93 (7) (2004) 075301.

[144] S.O. Diallo, J.V. Pearce, R.T. Azuah, F. Albergamo, H.R. Glyde, Condensate fraction and atomic kinetic energy of liquid He3-He4 mixtures, PRB 74 (14) (2006) 144503-+

[145] H.R. Glyde, R.T. Azuah, W.G. Stirling, Physical Review B 62 (2000) 14337-14349.

[146] R.N. Silver, Proceedings of the 1984 Workshop on High-Energy Excitations in Condensed Matter, vol. 2, in: Workshop held in Los Alamos, N. Mex. 13-15 Feb. 1984, pp. 345-648.

[147] D.M. Ceperley, R.O. Simmons, R.C. Blasdell, Physical Review Letters 77 (1996) 115-118.

[148] R.T. Azuah, W.G. Stirling, J. Mayers, I.F. Bailey, P.E. Sokol, Physical Review B 51 (1995) 6780-6783.

[149] Y. Wang, P.E. Sokol, Physical Review Letters 72 (1994) 1040-1043.

[150] D.N. Timms, R.O. Simmons, J. Mayers, Neutron measurements of the single-particle kinetic energies in solid neon, Physical Review B 67 (17) (2003) $172301-+$

[151] D.G. Narehood, M.K. Kostov, P.C. Eklund, M.W. Cole, P.E. Sokol, Deep inelastic neutron scattering of $\mathrm{H}_{2}$ in single-walled carbon nanotubes, Physical Review B 65 (23) (2002) 233401.

[152] D. Colognesi, C. Andreani, R. Senesi, Single-particle mean kinetic energy in low-density supercritical ${ }^{4}$ He, Europhysics Letters 50 (2000) $202-208$.

[153] R. Senesi, C. Andreani, D. Colognesi, A. Cunsolo, M. Nardone, Deep-inelastic neutron scattering determination of the single-particle kinetic energy in solid and liquid ${ }^{3} \mathrm{He}$, Physical Review Letters 86 (2001) 4584-4587. 
[154] J. Mayers, C. Andreani, D. Colognesi, Journal of Physics: Condensed Matter 9 (1997) 10639-10649.

[155] C. Andreani, C. Pantalei, R. Senesi, $\mathrm{He}_{4}$ adsorbed in cylindrical silica nanopores: effect of size on the single-atom mean kinetic energy, Physical Review B 75 (6) (2007) 064515-+.

[156] C. Andreani, C. Pantalei, R. Senesi, Mean kinetic energy of helium atoms in fluid ${ }^{3} \mathrm{He}$ and ${ }^{3} \mathrm{He}-{ }^{4} \mathrm{He}$ mixtures, Journal of Physics: Condensed Matter 18 (2006) 5587-5596.

[157] U. Bafile, M. Zoppi, F. Barocchi, R. Magli, J. Mayers, Physical Review Letters 75 (1995) 1957-1960.

[158] U. Bafile, M. Celli, M. Zoppi, J. Mayers, Physical Review B 58 (1998) 791-797.

[159] M.A. Adams, J. Mayers, O. Kirichek, R.B.E. Down, Measurement of the kinetic energy and lattice constant in hcp solid helium at temperatures 0.07 0.4 K, Physical Review Letters 98 (8) (2007) 085301-+.

[160] J. Mayers, F. Albergamo, D. Timms, Measurements of the atomic kinetic energy of ${ }^{4}$ He close to the superfluid transition, Physica B (Condensed Matter) $276(2000) 811-813$.

[161] L.P. Pitaevskii, S. Stringari, Bose-Einstein Condensation, Oxford University Press, Oxford, 2003.

[162] F. Mazzanti, A. Polls, J. Boronat, J. Casulleras, Physical Review Letters 92 (8) (2004) 085301.

[163] C. Cazorla, J. Boronat, Atomic kinetic energy momentum distribution, and structure of solid neon at zero temperature, Physical Review B 77 (2) (2008) 024310-+.

[164] C.J. Burnham, G.F. Reiter, J. Mayers, T. Abdul-Redah, H. Reichert, H. Dosch, On the origin of the redshift of the OH stretch in Ice Ih: evidence from the momentum distribution of the protons and the infrared spectral density, Physical Chemistry Chemical Physics (Incorporating Faraday Transactions) 8 (2006) 3966-+.

[165] J.A. Morrone, R. Car, Nuclear quantum effects in water, Physical Review Letters 101 (1) (2008) 017801

[166] M. Ceriotti, G. Bussi, M. Parrinello, Nuclear quantum effects in solids using a colored-noise thermostat, Physical Review Letters 103 (3) (2009) 030603.

[167] J.A. Morrone, V. Srinivasan, D. Sebastiani, R. Car, Proton momentum distribution in water: an open path integral molecular dynamics study, The Journal of Chemical Physics 126 (23) (2007) 234504. URL:http://link.aip.org/link/?JCP/126/234504/1.

[168] M. Ceriotti, G. Miceli, A. Pietropaolo, D. Colognesi, A. Nale, M. Catti, M. Bernasconi, M. Parrinello, Nuclear quantum effects in ab initio dynamics: theory and experiments for lithium imide, Physical Review B 82 (17) (2010) 174306.

[169] R.N. Silver, Proceedings of the 1984 Workshop on High-Energy Excitations in Condensed Matter, vol. 1, in: Workshop held in Los Alamos, N. Mex., 13-15 Feb. 1984, pp. 1-329.

[170] R.J. Newport, P.A. Seeger, W.G. Williams, Test measurements on a resonance filter spectrometer using electronvolt neutrons, Nuclear Instruments and Methods in Physics Research A 238 (1985) 177-179.

[171] S.W. Lovesey, C.D. Bowman, R.G. Johnson, Electron excitation in atoms and molecules by neutron-nucleus scattering, Zeitschrift fur Physik B (Condensed Matter) 47 (1982) 137-147.

[172] J.F. Cooke, J.A. Blackman, Calculation of neutron cross sections for interband transitions in semiconductors, Physical Review B 26 (1982) 4410-4420.

[173] J.F. Cooke, J.A. Blackman, T. Morgan, J.F. Cooke, J.A. Blackman, T. Morgan (Eds.), Band Structure Effects in the Electronic Properties of Solids Assessible in Neutron Scattering Studies, 1984.

[174] S.W. Lovesey, J. Mayers, Electrostatic neutron-electron scattering, Nuovo Cimento D Serie 7 (1986) 580-586.

[175] T. Morgan, J.A. Blackman, J.F. Cooke, Theoretical investigation of neutron scattering cross sections in Si and Ge, Physical Review B 33 (1986) 7154-7159.

[176] C. Stock, R.A. Cowley, W.J.L. Buyers, C.D. Frost, J.W. Taylor, D. Peets, R. Liang, D. Bonn, W.N. Hardy, Effect of the pseudogap on suppressing high energy inelastic neutron scattering in superconducting $\mathrm{YBa}_{2} \mathrm{Cu}_{3} \mathrm{O}_{6.5}$, Physical Review B 82 (17) (2010) 174505.

[177] H.A. Mook, D. Tocchetti, Neutron-scattering measurements of the generalized susceptibility $\chi(q, E)$ for Ni, Physical Review Letters 43 (1979) 2029-2032.

[178] K.N. Trohidou, J.A. Blackman, J.F. Cooke, Calculation of the high-energy spin-wave spectrum of hcp cobalt, Physical Review Letters 67 (1991) 2561-2564.

[179] J.A. Blackman, K.N. Trohidou, C. Petrillo, J.F. Cooke, S.W. Lovesey, F. Sacchetti, On the calculation of the dynamic structure factor from band structure models: application to iron, Journal of Physics C Solid State Physics 20 (1987) 3897-3910.

[180] S. Kern, C. Loong, G.H. Lander, Crystal-field transitions in f-electron oxides, Physical Review B 32 (1985) 3051-3057.

[181] A.I. Agafonov, Inelastic magnetic neutron scattering from inner filled shells of impurity atoms in metals, The European Physical Journal B (Condensed Matter and Complex Systems) 76 (2010) 271-275. doi:10.1140/epjb/e2010-00182-6. URL: http://dx.doi.org/10.1140/epjb/e2010-00182-6.

[182] A. Pietropaolo, R. Senesi, M. Tardocchi, C. Andreani, G. Gorini, Photon detectors for epithermal neutron scattering at high- $\omega$ and low- $q$, Physica B (Condensed Matter) 350 (2004) e857-e859.

[183] E. Perelli-Cippo, G. Gorini, M. Tardocchi, O. Cremonesi, C. Andreani, A. Pietropaolo, R. Senesi, Z.A. Bowden, N.J. Rhodes, E.M. Schooneveld, Development of the very low angle detector for epithermal neutron scattering at low momentum transfers, IEEE Transactions on Nuclear Science 52 (2005) 1092-1097.

[184] M. Tardocchi, A. Pietropaolo, C. Andreani, A. Bracco, A. D’Angelo, G. Gorini, S. Imberti, R. Senesi, N.J. Rhodes, E.M. Schooneveld, Cadmium-Zinc-Telluride photon detector for epithermal neutron spectroscopy-pulse height response characterisation, Nuclear Instruments and Methods in Physics Research A 526 (2004) 477-492.

[185] M. Tardocchi, A. Pietropaolo, C. Andreani, G. Gorini, S. Imberti, E. Perelli-Cippo, R. Senesi, N. Rhodes, E.M. Schooneveld, Comparison of Cadmium Zinc Telluride semiconductor and Yttrium aluminum perovskite scintillator as photon detectors for epithermal neutron spectroscopy, Nuclear Instruments and Methods in Physics Research A 567 (2006) 337-340.

[186] M.S. del Ro, A. Sodo, S. Eeckhout, T. Neisius, P. Martinetto, E. Dooryhe, C. Reyes-Valerio, Fe k-edge xanes of maya blue pigment, Nuclear Instruments and Methods in Physics Research Section B: Beam Interactions with Materials and Atoms 238 (1-4) (2005) 50-54. synchrotron Radiation in Materials Science - Proceedings of the 4th Conference on Synchrotron Radiation in Materials Science.

[187] T. Johansson, R. Akselsson, S. Johansson, X-ray analysis: elemental trace analysis at the 10-12 g level, Nuclear Instruments and Methods 84 (1) (1970) $141-143$.

[188] P. Grave, L. Lisle, M. Maccheroni, Multivariate comparison of icp-oes and pixe/pige analysis of east asian storage jars, Journal of Archaeological Science 32 (6) (2005) 885-896.

[189] A. Pietropaolo, G. Gorini, G. Festa, E. Reali, F. Grazzi, E.M. Schooneveld, A neutron resonance capture analysis experimental station at the isis spallation source, Applied Spectroscopy 64 (2010) 1068-1071(4). URL: http://www.ingentaconnect.com/content/sas/sas/2010/00000064/00000009/art00020.

[190] F. Grazzi, M. Celli, S. Siano, M. Zoppi, Preliminary results of the italian neutron experimental station INES at ISIS: archaeometric applications, Nuovo Cimento C Geophysics Space Physics C 30 (2007) 59-65.

[191] S. Imberti, W. Kockelmann, M. Celli, F. Grazzi, M. Zoppi, A. Botti, A. Sodo, M.L. Imperiale, M. de Vries-Melein, D. Visser, H. Postma, Neutron diffractometer INES for quantitative phase analysis of archaeological objects, Measurement Science and Technology 19 (3) (2008) 034003-+.

[192] G. Salvato, F. Aliotta, L. Bartoli, F. Grazzi, R.C. Ponterio, D. Tresoldi, C.S. Vasi, M. Zoppi, Neutron tomography at INES: first experimental results, Nuovo Cimento C Geophysics Space Physics C 31 (2008) 581-588.

[193] G. Gorini, The Ancient Charm Collaboration, Ancient Charm: a research project for neutron-based investigation of cultural-heritage objects, Nuovo Cimento C Geophysics Space Physics C 30 (2007) 47-58.

[194] M.C. Clarijs, V.R. Bom, C.W.E. van Eijk, A simple method for the analysis of neutron resonance capture spectra, Journal of Applied Physics 105 (6) (2009) 064910-+.

[195] E.M. Schooneveld, M. Tardocchi, G. Gorini, W. Kockelmann, T. Nakamura, E. Perelli Cippo, H. Postma, N. Rhodes, P. Schillebeeckx, The Ancient Charm Collaboration, Fast track communication: a new position-sensitive transmission detector for epithermal neutron imaging, Journal of Physics D Applied Physics 42 (15) (2009) 152003-+. 
[196] T. Belgya, Z. Kis, L. Szentmiklsi, Z. Kasztovszky, G. Festa, L. Andreanelli, M.De. Pascale, A. Pietropaolo, P. Kudejova, R. Schulze, T. Materna, A new pgai-nt setup at the nips facility of the budapest research reactor, Journal of Radioanalytical and Nuclear Chemistry 278 (2008) 713-718. doi:10.1007/s10967008-1510-0. URL: http://dx.doi.org/10.1007/s10967-008-1510-0.

[197] I.S. Anderson, R. Mcgreevy, H.Z. Biliheux, Neutron Imaging and Applications, Springer, New York, 2009.

[198] E. Normand, Single event upset at ground level, IEEE Transactions on Nuclear Science 43 (1996) 2742-2750.

[199] J.F. Ziegler, W.A. Lanford, The effect of sea level cosmic rays on electronic devices, Journal of Applied Physics 52 (1981) $4305-4312$.

[200] F. Wrobel, J. Gasiot, F. Saigné, A.D. Touboul, Effects of atmospheric neutrons and natural contamination on advanced microelectronic memories, Applied Physics Letters 93 (6) (2008) 064105-+.

[201] E. Normand, T.J. Baker, Altitude and latitude variations in avionics SEU and atmospheric neutron flux, IEEE Transactions on Nuclear Science 40 (1993) $1484-1490$.

[202] IEC TS 62396, Standard for the Accommodation of Atmospheric Radiation Effects via Single Event Effects within Avionics Electronic Equipment, Part 1, 2005. URL: http://www.iec.ch/.

[203] JEDEC Standard JESD89A Measurement and Reporting of Alpha Particle and Terrestrial Cosmic Ray-Induced Soft Errors in Semiconductor Devices, 2006. URL: http://www.jedec.org.

[204] M. Violante, L. Sterpone, A. Manuzzato, S. Gerardin, P. Rech, M. Bagatin, A. Paccagnella, C. Andreani, G. Gorini, A. Pietropaolo, G. Cardarilli, S. Pontarelli, C. Frost, A new hardware/software platform and a new 1/E neutron source for soft error studies: testing FPGAs at the ISIS facility, IEEE Transactions on Nuclear Science 54 (2007) 1184-1189.

[205] C. Andreani, A. Pietropaolo, A. Salsano, G. Gorini, M. Tardocchi, A. Paccagnella, S. Gerardin, C.D. Frost, S. Ansell, S.P. Platt, Facility for fast neutron irradiation tests of electronics at the ISIS spallation neutron source, Applied Physics Letters 92 (11) (2008) 114101-+.

[206] Description of the Chipir beam line at ISIS, URL: http://www.isis.stfc.ac.uk/instruments/Chipir/.

[207] V.P. Eismont, A.V. Prokofyev, A.N. Smirnov, Thin-film breakdown counters and their applications (review), Radiation Measurements 25 (1-4) (1995) 151-156. nuclear Tracks in Solids. URL: http://www.sciencedirect.com/science/article/B6TVS-3YF49K5-16/2/ 69a0900d0e653deb0d51258f9d422cec.

[208] A. Pietropaolo, G. Verona Rinati, C. Verona, E.M. Schooneveld, M. Angelone, M. Pillon, A single-crystal diamond-based thermal neutron beam monitor for instruments at pulsed neutron sources, Nuclear Instruments and Methods in Physics Research A 610 (2009) 677-681.

[209] M. Rebai, C. Andreani, A. Fazzi, C.D. Frost, L. Giacomelli, G. Gorini, E. Milani, E. Perelli Cippo, A. Pietropaolo, G. Prestopino, E. Schooneveld, M. Tardocchi, C. Verona, G. Verona Rinati, Fission diamond detector tests at the ISIS spallation neutron source, Nuclear Physics B Proceedings Supplements 215 (2011) 313-315.

[210] L. Giacomelli, C. Andreani, A. Fazzi, C.D. Frost, G. Gorini, E. Perelli Cippo, A. Pietropaolo, M. Rebai, H. Schuhmacher, M. Tardocchi, C. Verona, G. Verona Rinati, A. Zimbal, Diamond detectors for fast neutron irradiation experiments, Nuclear Physics B Proceedings Supplements 215 (2011) $242-246$.

[211] A. Pietropaolo, C. Andreani, M. Rebai, L. Giacomelli, G. Gorini, E. Perelli Cippo, M. Tardocchi, A. Fazzi, G. Verona Rinati, C. Verona, M. Marinelli, E. Milani, C.D. Frost, E.M. Schooneveld, Fission diamond detectors for fast-neutron ToF spectroscopy, Europhysics Letters 94 (2011) 62001-+.

[212] A. Pietropaolo, C. Andreani, M. Rebai, L. Giacomelli, G. Gorini, E. Perelli Cippo, M. Tardocchi, A. Fazzi, G. Verona Rinati, C. Verona, M. Marinelli, E. Milani, C.D. Frost, E.M. Schooneveld, Single-crystal diamond detector for time-resolved measurements of a pulsed fast-neutron beam, Europhysics Letters 92 (2010) 68003-+.

[213] R. Newport, J. Penfold, W. Williams, Electron volt spectroscopy on a pulsed neutron source, Nuclear Instruments and Methods in Physics Research 224 (1-2) (1984) 120-132. URL: http://www.sciencedirect.com/science/article/B73DP-478HG8P-8N/2/8f5e77a35b6cba52765abc2fc141406e.

[214] P. Postorino, F. Fillaux, J. Mayers, J. Tomkinson, R.S. Holt, The anisotropy of the proton momentum distribution in $\mathrm{KHCO}_{3}$ : a deep inelastic neutron scattering study, The Journal of Chemical Physics 94 (1991) 4411-4415.

[215] B. Rosi-Schwartz, J.A. Blackman, G.R. Mitchell, J. Mayers, Hydrogen momentum distribution in anisotropic rigid-chain polymers, Journal of Physics: Condensed Matter 4 (1992) 5665-5674.

[216] J. Mayers, Measurement of the proton wave function in molecular hydrogen by neutron Compton scattering, Physical Review Letters 71 (1993) 1553-1556.

[217] R. Senesi, A. Pietropaolo, A. Bocedi, S.E. Pagnotta, F. Bruni, Proton momentum distribution in a protein hydration shell, Physical Review Letters 98 (13) (2007) 138102-+

[218] S.E. Pagnotta, F. Bruni, R. Senesi, A. Pietropaolo, Quantum behavior of water protons in protein hydration shell, Biophysical Journal 96 (2009) 1939-1943.

[219] A. Pietropaolo, G. Gorini, G. Festa, C. Andreani, M.P. de Pascale, E. Reali, F. Grazzi, E.M. Schooneveld, A silicon photomultiplier readout for time of flight neutron spectroscopy with $\gamma$-ray detectors, Review of Scientific Instruments 80 (9) (2009) 095108-+.

[220] L.K. Mansur, J.R. Haines, Status of the Spallation Neutron Source with focus on target materials, Journal of Nuclear Materials 356 (2006) 1-15.

[221] J. Wei, H. Chen, Y. Chen, Y. Chen, Y. Chi, C. Deng, H. Dong, L. Dong, S. Fang, J. Feng, S. Fu, L. He, W. He, Y. Heng, K. Huang, X. Jia, W. Kang, X. Kong, J. Li, T. Liang, G. Lin, Z. Liu, H. Ouyang, Q. Qin, H. Qu, C. Shi, H. Sun, J. Tang, J. Tao, C. Wang, F. Wang, D. Wang, Q. Wang, S. Wang, T. Wei, J. Xi, T. Xu, Z. Xu, W. Yin, X. Yin, J. Zhang, Z. Zhang, Z. Zhang, M. Zhou, T. Zhu, China spallation neutron source: design, R\&D, and outlook, Nuclear Instruments and Methods in Physics Research A 600 (2009) 10-13.

[222] F. Maekawa, M. Harada, K. Oikawa, M. Teshigawara, T. Kai, S. Meigo, M. Ooi, S. Sakamoto, H. Takada, M. Futakawa, T. Kato, Y. Ikeda, N. Watanabe, T. Kamiyama, S. Torii, R. Kajimoto, M. Nakamura, First neutron production utilizing J-PARC pulsed spallation neutron source JSNS and neutronic performance demonstrated, Nuclear Instruments and Methods in Physics Research A 620 (2010) 159-165. 